\title{
3D laser imaging and modeling of iron meteorites and tektites
}

\author{
by
}

Christopher A. Fry

A thesis submitted to the Faculty of Graduate and Postdoctoral Affairs in partial fulfillment of the requirements for the degree of

Master of Science

in

Earth Science

Carleton University Ottawa, Ontario

C2013, Christopher Fry 


\begin{abstract}
3D laser imaging is a non-destructive method devised to calculate bulk density by creating volumetrically accurate computer models of hand samples. The focus of this research was to streamline the imaging process and to mitigate any potential errors. 3D laser imaging captured with great detail ( $\approx 30$ voxel $/ \mathrm{mm}^{2}$ ) surficial features of the samples, such as regmaglypts, pits and cut faces. Densities from 41 iron meteorites and 9 splash-form Australasian tektites are reported here. The laser-derived densities of iron meteorites range from 6.98 to $7.93 \mathrm{~g} / \mathrm{cm}^{3}$. Several suites of meteorites were studied and are somewhat heterogeneous based on an average $2.7 \%$ variation in inter-fragment density. Density decreases with terrestrial age due to weathering. The tektites have an average laser-derived density of $2.41 \pm 0.11 \mathrm{~g} / \mathrm{cm}^{3}$. For comparison purposes, the Archimedean bead method was also used to determine density. This method was more effective for tektites than for iron meteorites.
\end{abstract}




\section{Acknowledgements}

A M.Sc. thesis is a large undertaking that cannot be completed alone. There are several individuals who contributed significantly to this project. I thank Dr. Claire Samson, my supervisor, without whom this thesis would not have been possible. Her guidance and encouragement is largely the reason that this project was completed. Her patient reading and constructive comments helped this thesis achieve its full potential. I would like to extend my deepest gratitude to Dr. Richard Herd of the Natural Resources Canada, Dr. Phillip McCausland of Western University, Dr. Chris Herd of the University of Alberta and Dr. Dan Britt of the University of Central Florida for their generous loan of the iron meteorite samples used in this study. By the same token, I would like to thank Dr. Mel Stauffer and Dr. Sam Butler for the use of the splash-form tektites. At the same time, Dr. Butler, Dr. McCausland, and Dr R. Herd provided invaluable knowledge for which I am deeply grateful. Their discussions were highly beneficial, and aided in understanding the results of this study. Maxim Ralchekno and Tara McLeod provided countless hours of their time as secondary operators in the methods used. Dave Melanson was very kind and took a number of the digital photographs displayed in this work. Lastly,Dr. Jason Mah who took time out of a very busy schedule to help me perform the PCA analysis and to provide guidance on its interpretation. 
Table of Contents

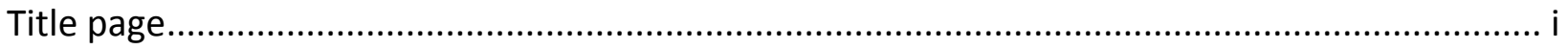

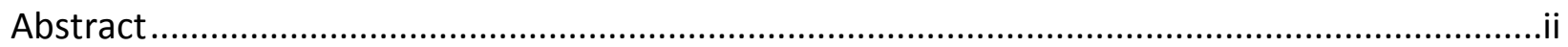

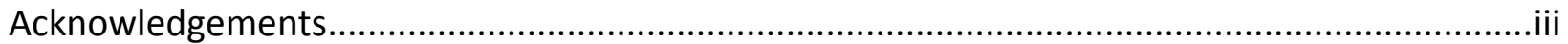

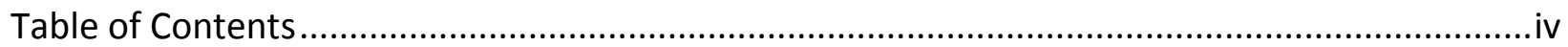

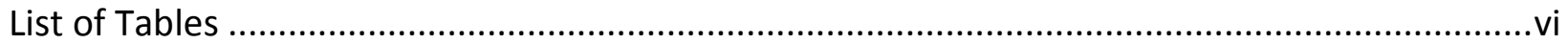

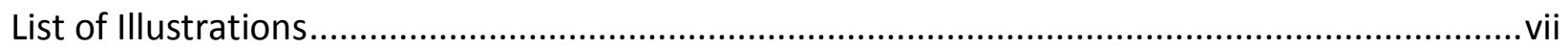

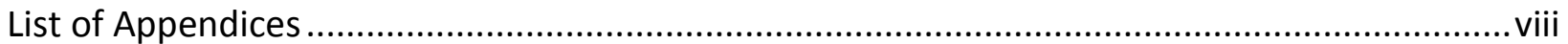

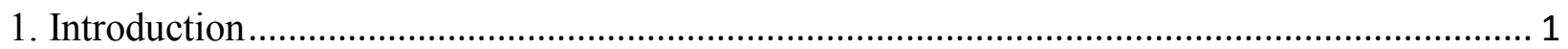

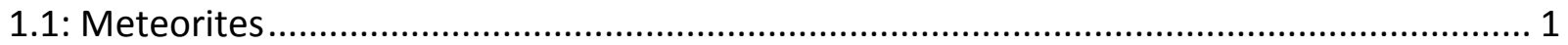

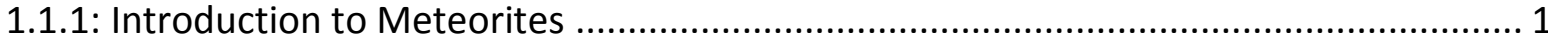

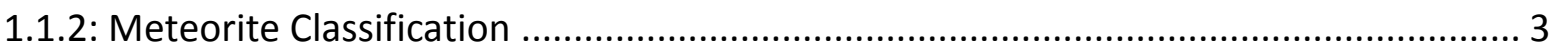

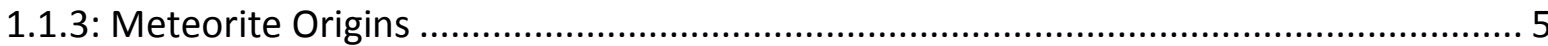

1.1.4: Meteorite Morphology ............................................................................................... 7

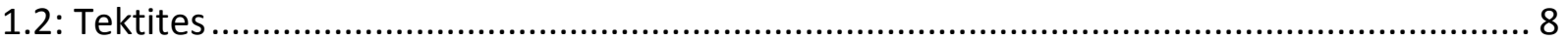

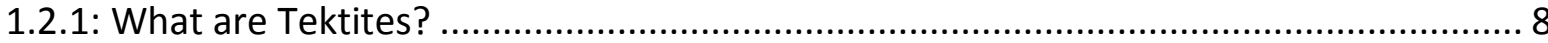

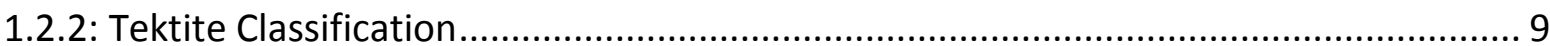

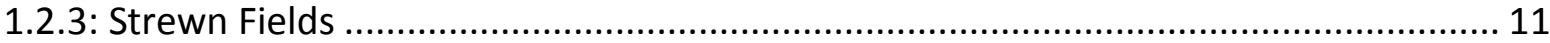

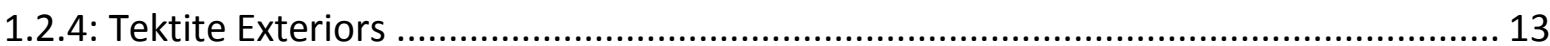

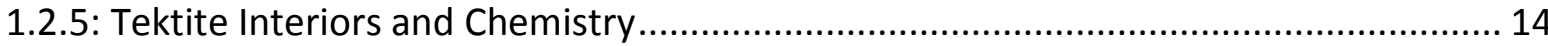

1.3: Physical properties of meteorites and tektites .......................................................... 16

1.3.1: Introduction to Physical Properties .......................................................................... 16

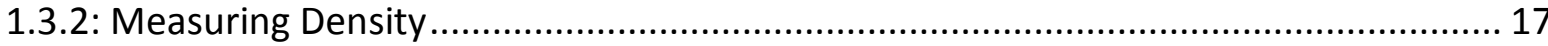

1.3.3: Meteorite Densities in the Literature................................................................... 19

1.3.4: Tektite Densities in the Literature .................................................................. 21

1.4: Research Objectives and Thesis Structure ............................................................. 21

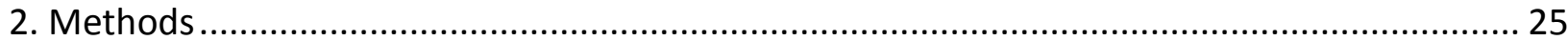

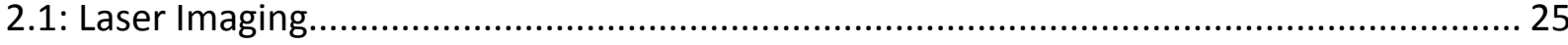

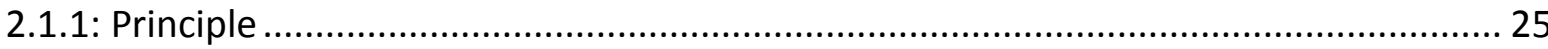

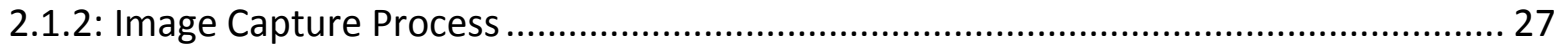

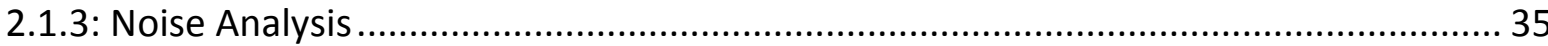




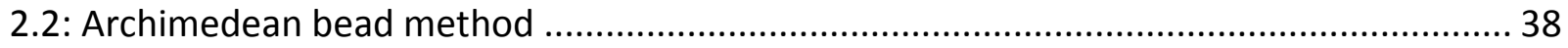

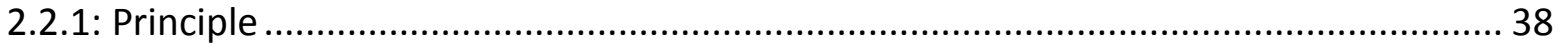

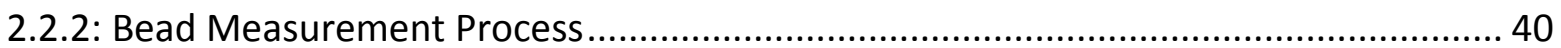

3. Iron Meteorites .............................................................................................................. 43

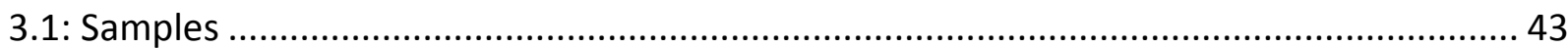

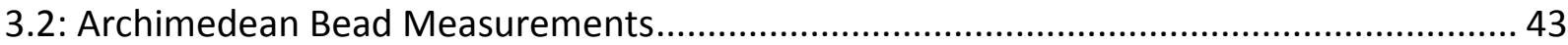

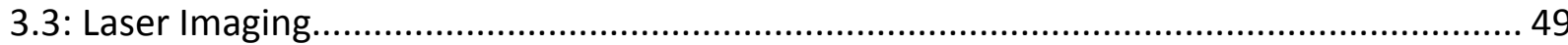

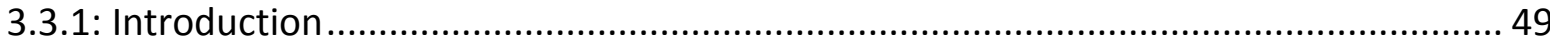

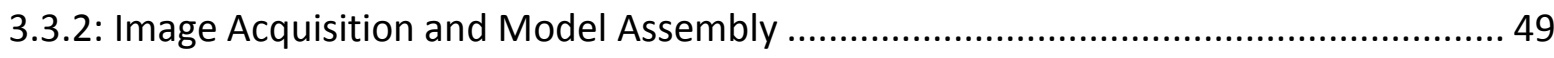

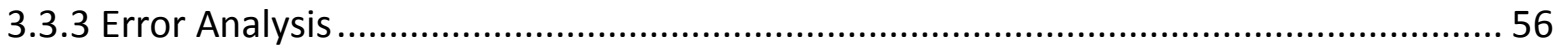

3.4: Comparison between densities derived using the Archimedean Bead Method and Laser

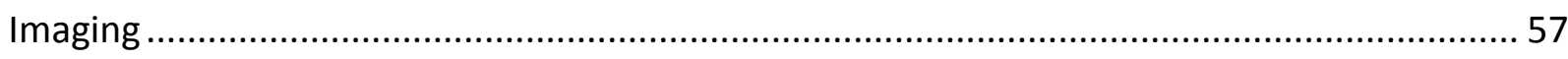

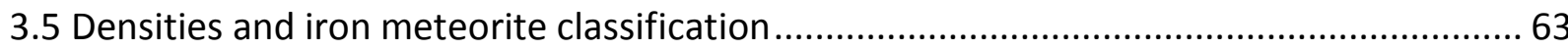

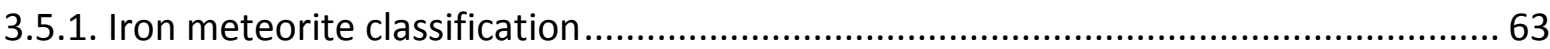

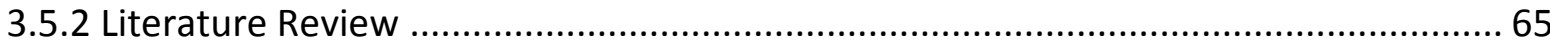

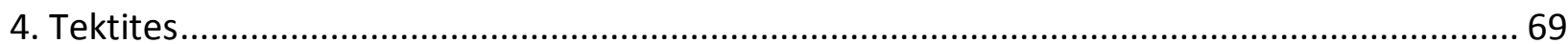

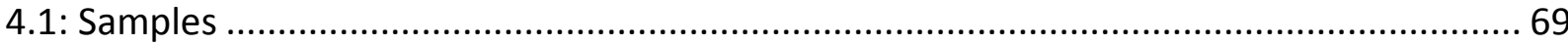

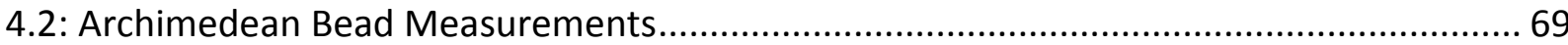

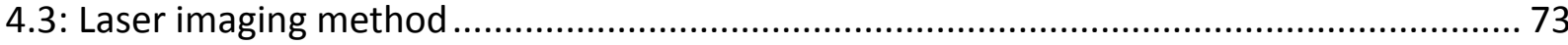

4.3.1: Image Acquisition and Assembly ............................................................................. 73

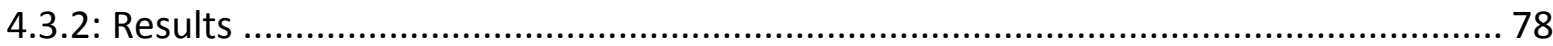

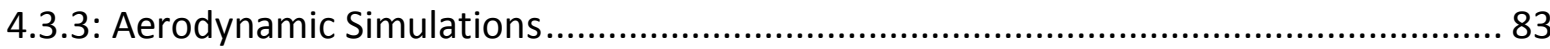

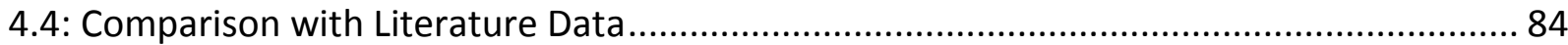

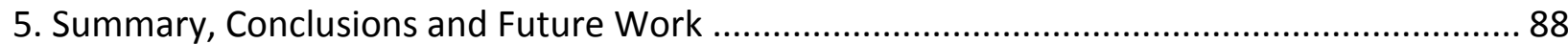

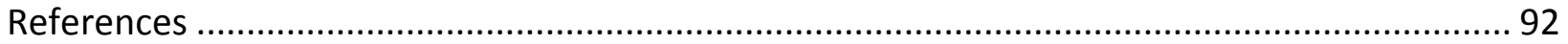




\section{List of Tables}

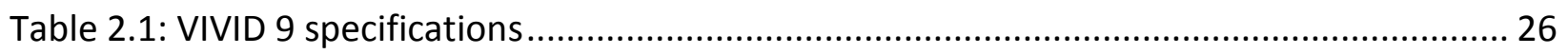

Table 3.1: Data on iron meteorite samples ............................................................................. 46

Table 3.2: Archimedean bead results for iron meteorites ................................................... 46

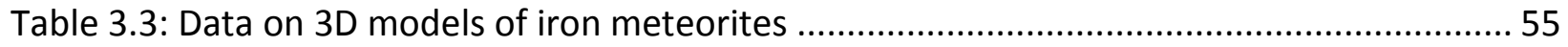

Table3.4: Comparison in laser and bead derived densities of iron meteorites ......................... 62

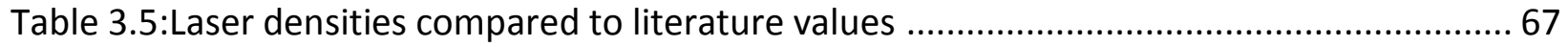

Table 4.1: Archimedean bead density measurements on tektites......................................... 70

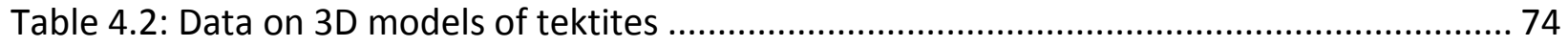

Table 4.3: Moments of inertia and rotation period of tektite samples................................... 86 


\section{List of Illustrations}

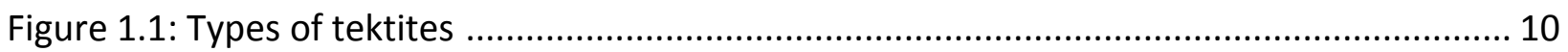

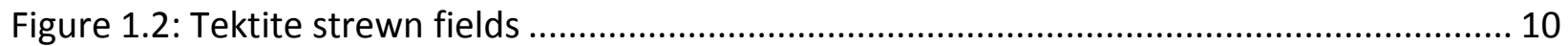

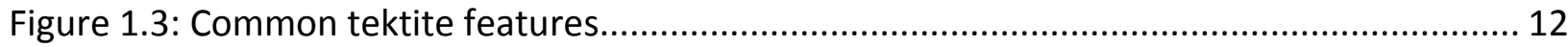

Figure 2.1: Schematic diagram of VIVID 9i laser camera ......................................................... 28

Figure 2.2: Arrangement of 3D laser camera system. ....................................................... 28

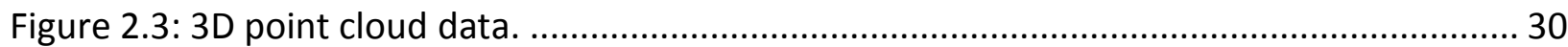

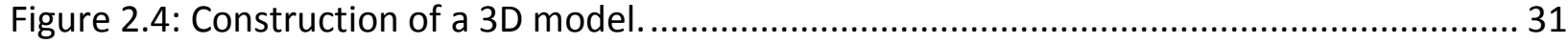

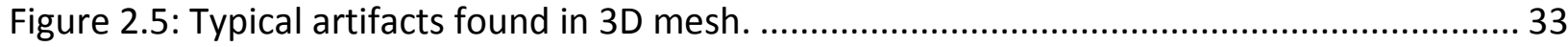

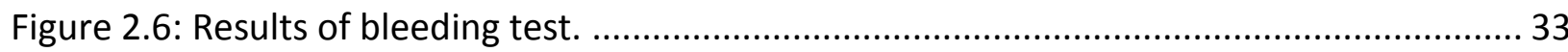

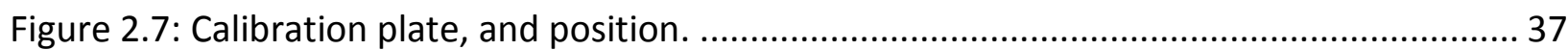

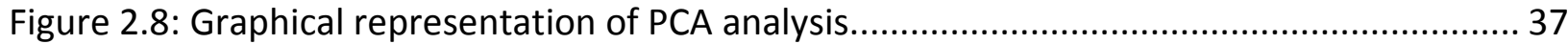

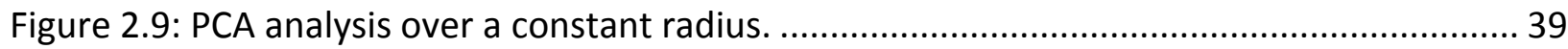

Figure 2.10: PCA analysis over a variable radius ................................................................... 39

Figure 2.11: Example of the Archimedean bead method....................................................... 42

Figure 3.1: Examples of iron meteorites examined.............................................................. 44

Figure 3.2: Comparison of samples studied to known meteorites.......................................... 44

Figure 3.3: Error associated with the bead measurements ................................................... 48

Figure 3.4: Fragment of the Odessa meteorite ................................................................. 48

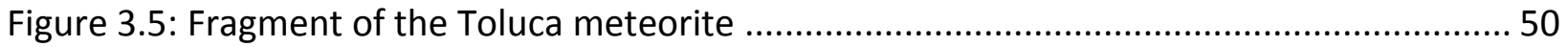

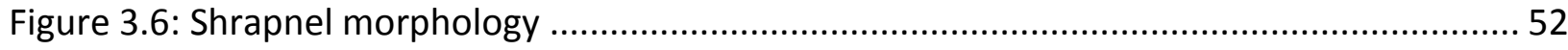

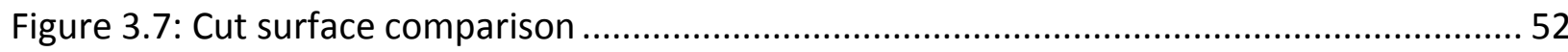

Figure 3.8: Comparison between shape and size parameters and coefficient of variation.... 58-59

Figure 3.9: Comparison between density measurements derived by both methods................. 60

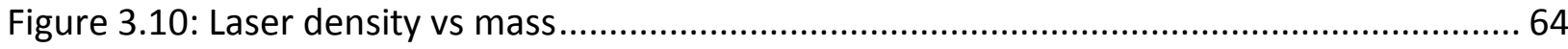

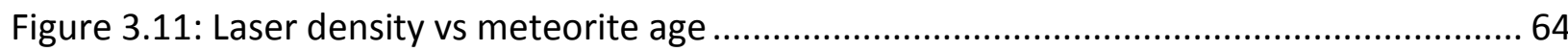

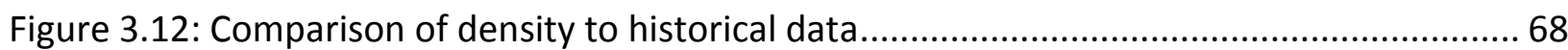

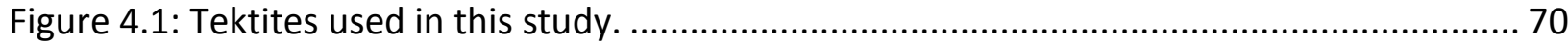

Figure 4.2: Tektite Archimedean bead data for two operators............................................ 72

Figure 4.3: Sclieren present in 3D mesh. ......................................................................... 72

Figure 4.4: 3D polygonal model with and without smoothing................................................. 74

Figure 4.5: Watertightness of tektite and meteorite models.............................................. 77

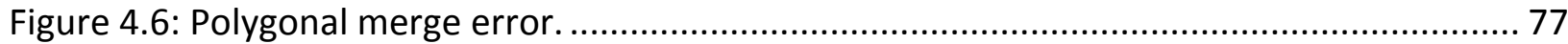

Figure 4.7: Comparison of density to size and shape of tektite. ..................................... 79-80

Figure 4.8: Comparison between density derived by both methods ....................................... 82

Figure 4.9: Principle rotation axes of two tektite .............................................................. 82

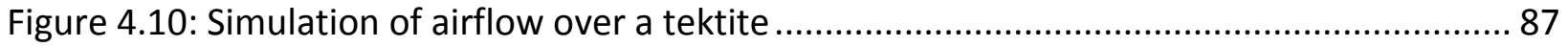




\section{List of Appendices}

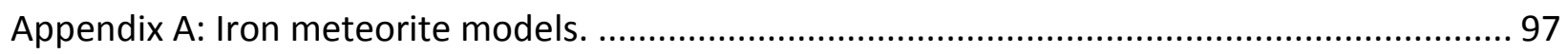

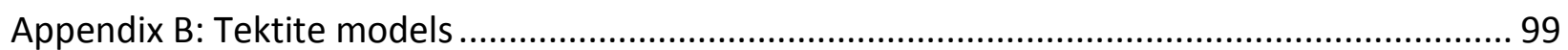

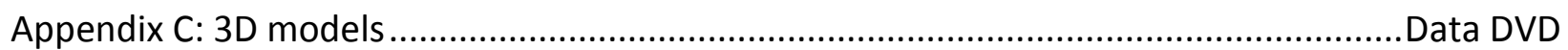




\section{Introduction}

\section{1: Meteorites}

\subsection{1: Introduction to Meteorites}

On February 15, 2013, residents in Chelyabinsk, Russia spotted a brilliant light plummeting from the sky. What people were seeing was a meteor as it descended through the Earth's atmosphere from space. Most meteors are much less spectacular, with millions of tiny particles falling to the Earth each day unnoticed. These particles (diameter $\approx 0.001-1 \mathrm{~mm}$ ) are essentially interstellar dust, that is, fine debris from collisions between larger bodies (Norton, 2002). Some materials striking the Earth from space are large enough to be noticed and survive atmospheric entry. What is recovered is a meteorite, a lump of extraterrestrial rock that does not fit into a regular geological context (McSween, 1999).

Meteorites have been striking the Earth for its entire history. The impact rate of meteorites on the Earth has been steadily declining since the period of 3.8-3.1 Ga when it was particularly intense (Hartman et al., 2007). The Earth, as a dynamic body, has hidden the signs of fallen meteors over the eons, but some evidence still remains. Sometimes, a meteorite impact will leave a crater in the ground, such as the classic "Meteor Crater" found by Daniel Barringer in the Arizona desert. In other cases, such as the Manicouagan Crater, erosion has erased most traces of the impact and only the barest outline, visible now only on satellite or aerial imagery, is left. The effects of meteorite impacts can be seen on inert, pockmarked bodies like the Moon, where there is no current activity to hide (e.g. young lava flows) or erase (e.g. flowing water) the craters. 
Meteorites typically inherit the name of the place or area in which, or close to, where they fall, or close to it. Should a meteorite fall to Earth and be retrieved in Ottawa, it would be called the Ottawa meteorite. It would also be called a "fall". A meteorite whose fall was not observed, and that has been resting on the Earth for some time, is known as a "find".

The study of meteorites is called meteoritics, and this thesis is a contribution to this multidisciplinary science. Over the past few decades, meteoritics has been a field of research that has been growing, with the advent of new analytical techniques and equipment, and the increase in numbers of meteorites available to researchers (Norton, 2002). The subject has come a long way since its inception in the late 1700's, when many scientists could not believe that this material had come from space (McSween, 1999).

The study of meteorites can help to provide insight into the past of the solar system. As will be covered in the next section, iron meteorites, one of the two materials studied, represent the interior of a large, differentiated body. Studying physical properties of meteorites, along with chemistry and mineralogy, can be used to build a link between meteorites and potential parent bodies. Density and porosity can be used as well to obtain more information about the internal structure of these bodies (Kohout et al., 2008). Measuring these properties non-destructively is important to preserve samples for future studies. Unlike terrestrial samples, the availability of iron meteorites depends on the chance of them falling to Earth and surviving atmospheric entry. The viability of a recent method to determine density, 3D laser imaging, will be investigated in this thesis for iron meteorites and extended to the study of tektites, an impact related material. 


\subsection{2: Meteorite Classification}

A meteorite, the recovered fragment of a meteor, can be classified within one of a few broad groups that are defined by their composition. These groups are: the stony meteorites, composed almost entirely of silicate and oxide material; the stony-iron meteorites, composed of equal parts metal and silicate; and the iron meteorites, composed almost entirely of nickeliron minerals. These groups can be further subdivided, based mostly on chemistry. Meteorites are mostly composed of minerals similar to those found on Earth (e.g. silicates, such as olivine or pyroxene) (Norton, 2002). There are also at least 20 minerals that occur in meteorites that are not found on Earth (such as schreibersite, an iron nickel phosphide), and approximately two dozen secondary minerals (such as goethite) that are produced by weathering in the terrestrial environment (Buchwald, 1975). Others, like the mineral troilite, are common in meteorites, but rare on Earth, with polymorphs being found instead.

\section{Stony meteorites}

Stony meteorites can be further divided into two categories: chondrites and achondrites. Chondrites get their name from numerous small inclusions, called chondrules, which are a part of their petrologic fabric. Chondrules are millimeter-sized igneous silicate particles, composed primarily of olivine, pyroxene and glass. Of note is that these primitive particles are thought to have formed in the solar nebula, and thus provide insight to conditions in the early solar system (Hewins, 1997). Chondrites are composed of olivines, pyroxenes and feldspar minerals. They can also possess inclusions of iron minerals (taenite, kamacite and troilite), sulfide and spinel (McSween, 1999). 
Achondrites, on the other hand, do not possess chondrules. They have an igneous origin, forming from the crystallization of magma (McSween, 1999). Their classification scheme is based first on their origin (asteroidal, martian or lunar) and then on their mineral and chemical composition (Norton, 2002). In general, achondrites are composed of varying amounts of pyroxene, olivine and feldspar with very little iron (Norton, 2002).

In between the stony meteorites and the iron meteorites is a group called the stony-irons. They contain subequal amounts of silicates, largely olivine, and Fe-Ni. These meteorites can be split into two groups, based on their structure. In one group (the pallasites), metal forms a mesh within which the silicate(almost solely magnesian olivine) rests, while the other group(the mesosiderites) includes polymict breccias of plagioclase, pyroxene and metal, that have undergone different degrees of thermal metamorphism and recrystallization (Hutchinson, 2004).

Iron Meteorites

Of particular relevance to this thesis are iron meteorites which account for approximately $4 \%$ of falls and $40 \%$ of finds (Norton, 2002). The percentage of finds versus falls is skewed as iron meteorites typically do not degrade as fast as stony meteorites when subjected to the terrestrial environment.

Iron meteorites are essentially iron-nickel alloys, with those two elements consisting, on average, of $>98 \mathrm{wt} \%$ (often iron is at least $90 \mathrm{wt} \%$ of the meteorite) of their composition, with everything else occurring in trace amounts (Wasson and Kallemeyn, 2002; Buchwald, 1975). The predominant minerals are the high nickel mineral (20-45\%) taenite, and the low nickel 
mineral (<7\%) kamacite. Their crystallization depends on the amount of nickel present in the alloy, and the cooling temperature. Kamacite and taenite often coexist together within the meteorite, and combined will make up the bulk of the mass. Also present is troilite (an iron sulfide), graphite, and plessite, an intergrowth mixture of kamacite and taenite (McSween, 1999).

There are two classification schemes for iron meteorites. The first, based on the structure of the sample, is divided into three categories; hexahedrites, octahedrites and ataxites. Cut and etched surfaces of octahedrites display a very distinctive interlocking texture known as the Widmanstätten pattern (Figure 3.5C), formed by the intergrowth of cubic kamacite and taenite crystals. Each of the three categories display thin lamellae, known as Neumann lines, Widmanstätten pattern of varying thickness, and no structure at all, respectively. The chemical scheme separates iron meteorites into a number of groups and subgroups, based on nickel and trace element (in ppm) compositions (Norton, 2002). Trace elements include gallium, germanium and iridium. Combining the two classification schemes divides the iron meteorites into several distinct groups that likely represent different parent bodies (Norton, 2002).

\subsection{3: Meteorite Origins}

Chondrite mineral assemblages are dated as some of the oldest items in the solar system, and have remained unchanged, garnering them the title of primitive material. They serve as a window into the early solar system (Norton, 2002). Achondrites and iron meteorites on the other hand are differentiated, having undergone some partial or total melting since their time of formation. 
Meteorites are generally agreed to be fragments of rock that have broken off of an asteroid, or other parent body, at some point in the past (Norton, 2002). The formation of an asteroid is likely similar to that of the Earth, where material was accreted and then differentiated. A plausible scenario could be that the original chondritic, or potentially pre-chondritic material, condensed through collisional growth to form a body $100-1000 \mathrm{~km}$ in diameter. The latent heat of condensation melted this material, to produce a layered body (Goldstein et al., 2009). Most iron meteorites are differentiated objects that represent the core of such a body (Norton, 2002). While some iron meteorites show signs of having formed from a slowly cooling magma, others have a non-magmatic origin, believed by some researchers to be the result of impact generated melts (Wasson and Kallemeyn, 2002). These same impacts split open the large bodies, into the asteroids of today, exposing the iron core to further impact. The parents of chondrites are likely bodies too small for differentiation (McSween, 1999). Achondrites represent the silicate material surrounding the metallic core of a body large enough to have differentiated. Some of them are impact ejecta from Mars or the Moon, while others come from asteroids (Norton, 2002). Overall, there is estimated to be at least 100 asteroidal parent bodies, 60 of which could have produced iron meteorites (Burbine et al., 2002; Goldstein et al., 2009). 


\subsection{4: Meteorite Morphology}

The size of meteorites is extremely variable. They can be on the scale of millimeters, or in the rare case of the Hoba iron, multiple meters in diameter. The common size, however, is about that of a baseball (around $8 \mathrm{~cm}$ in diameter) (Norton, 2002).

The shape of meteorites may also vary greatly. They are ablated as they pass through the atmosphere. Pending on how long and how this is occurring, it may smooth their surfaces. Uneven ablation of surfaces can produce sides that have been rounded while others are jagged and fragmented. Cracks and crevasses may occur during this process. Turbulent airstreams that are produced during the ablation process can serve to melt the meteorite, leaving rounded cavities, known as regmaglypts, behind. Regmaglypts (seen in Appendix A) resemble thumb-like imprints on the meteorite surface, and are often well developed on iron meteorites (Buchwald, 1975). The cavities may be enhanced by the differential melting of softer minerals (Norton, 2002).They may only cover part of a meteorite fragment, depending on its orientation while airborne. Also produced by ablation are fusion crusts, dark coatings where the outer part of the sample has melted and hardened. Often, weathering will remove this crust from older iron samples, and replace it by terrestrially produced magnetite (Norton, 2002). In younger samples, however, the fusion crust is seen to be more complex for iron meteorites than for stony meteorites, consisting of mixed melts of fully and partially oxidized metal (Buchwald, 1975).

Finally, some meteorites, such as Sikhote-Alin (Appendix A), exploded on impact producing jagged and distorted shrapnel-like samples. 
Another important characteristic of iron meteorite surfaces is weathering. Iron meteorites have not been in contact with oxygen and water for most of their history, and are quickly susceptible to their effects once arriving on Earth. A number of different oxides can form, depending on the conditions, leaving a rust-colored coating on their outer surface. A perfect example is the Whitecourt meteorite (Appendix A), which has been heavily oxidized. Some weathered irons, including the Odessa meteorite included in this study, are found to have accumulated chlorine in a form of chemical weathering that produces lawrencite, an iron chloride (Buchwald, 1975). It is a green-brown mineral that will form along the crystal boundaries of kamacite and taenite.

\section{2: Tektites}

\subsection{1: What are Tektites?}

Meteoritic impacts can have a large effect on the surrounding area, sometimes creating craters, shatter cones and/or natural glass lumps, known as tektites (Glass and Koberl, 2006). Tektites can be distinguished from other natural glasses, such as obsidian, by their reaction to heating, their petrography and their unique surface sculpturing (Glass, 1974).

The exact origin of tektites and their mode of formation is a topic that has been discussed at length for decades. Some researchers thought that they had been produced by lunar volcanism or impact and came to Earth as a shower, though there was geochemical evidence otherwise (O’Keefe, 1976; O’Keefe, 1994; Taylor and Koeberl, 1994). Some simply favored an extraterrestrial parent, such as a comet, that grazed the atmosphere (before impact) and parted with melted fragments (loess) which then fell to Earth (Adams and Huffaker, 1964). 
Other origin ideas included formation from lightning strikes, such as in the case of fulgurites, residue from the evaporation of silica-rich waters, or volcanic bombs (Barnes, 1967). It is now generally agreed that tektites are produced by the rapid melting and cooling of shock melt during a terrestrial impact (e.g. Glass, 1990; Koeberl, 1994; Dressler and Reimold, 2001).

\subsection{2: Tektite Classification}

There are several types of tektites (Figure 1.1). This study will be dealing with what are known as splash-form tektites (Appendix B), which are the most common. The name "splashform" comes from their general shapes resembling hardened blobs of liquid. They can exhibit a variety of shapes such as ellipsoids, flattened discs, dumbbells, rods, teardrops and pseudo tori. This variety of shapes is a result of airborne molten rock spinning at different rates while cooling and solidifying (Stauffer and Butler, 2010). Other tektite forms observed are microtektites, Muong Nong and ablated forms. The microtektites are under $1 \mathrm{~mm}$ in size, have rotation shapes that greatly resemble those of the splash-forms, and possess many of the same surface features (Glass, 1974). Muong Nong tektites are chunks that have been broken from a layered mass. The ablated-form tektites have been shaped by a secondary aerodynamic force (ablation) after the main splash-form shapes have been produced, but were still airborne; part of their surface has undergone an additional melting phase, due to atmospheric friction, producing a flange (Stauffer and Butler, 2010). Tektites may have undergone secondary processes that modify their shape, such as fragmentation (in the air, upon impact or during sedimentary transport), degassing, chemical corrosion or surface abrasion. 

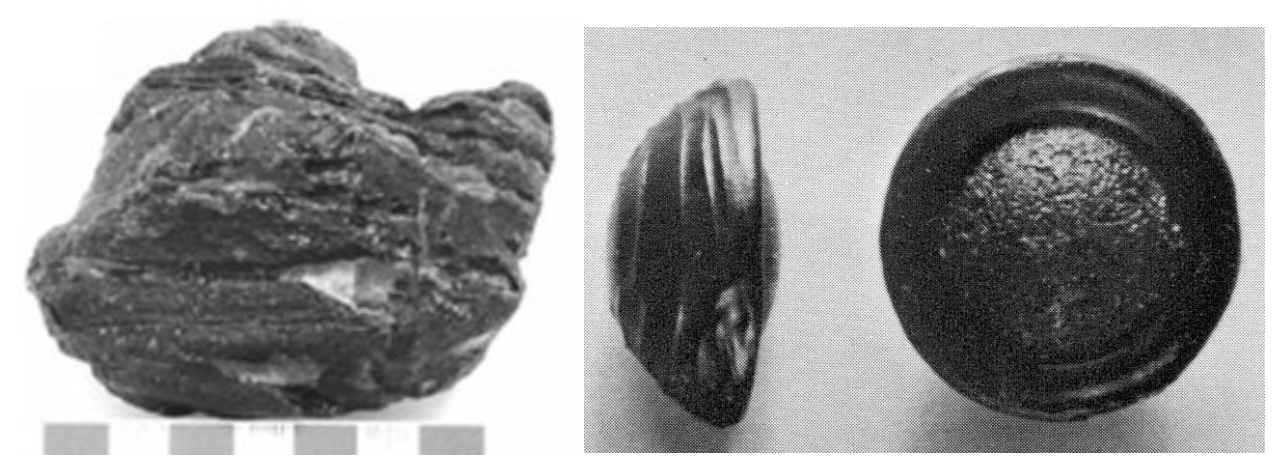

Figure 1.1: Two of the three different types of tektites. Left: Muong Nong tektite, from Stauffer and Butler (2010). Scale is in centimeters. Right: An ablated tektite, in the shape of a button, from O'Keefe (1976). Refer to Appendix 2 for a variety of splash-form tektites.

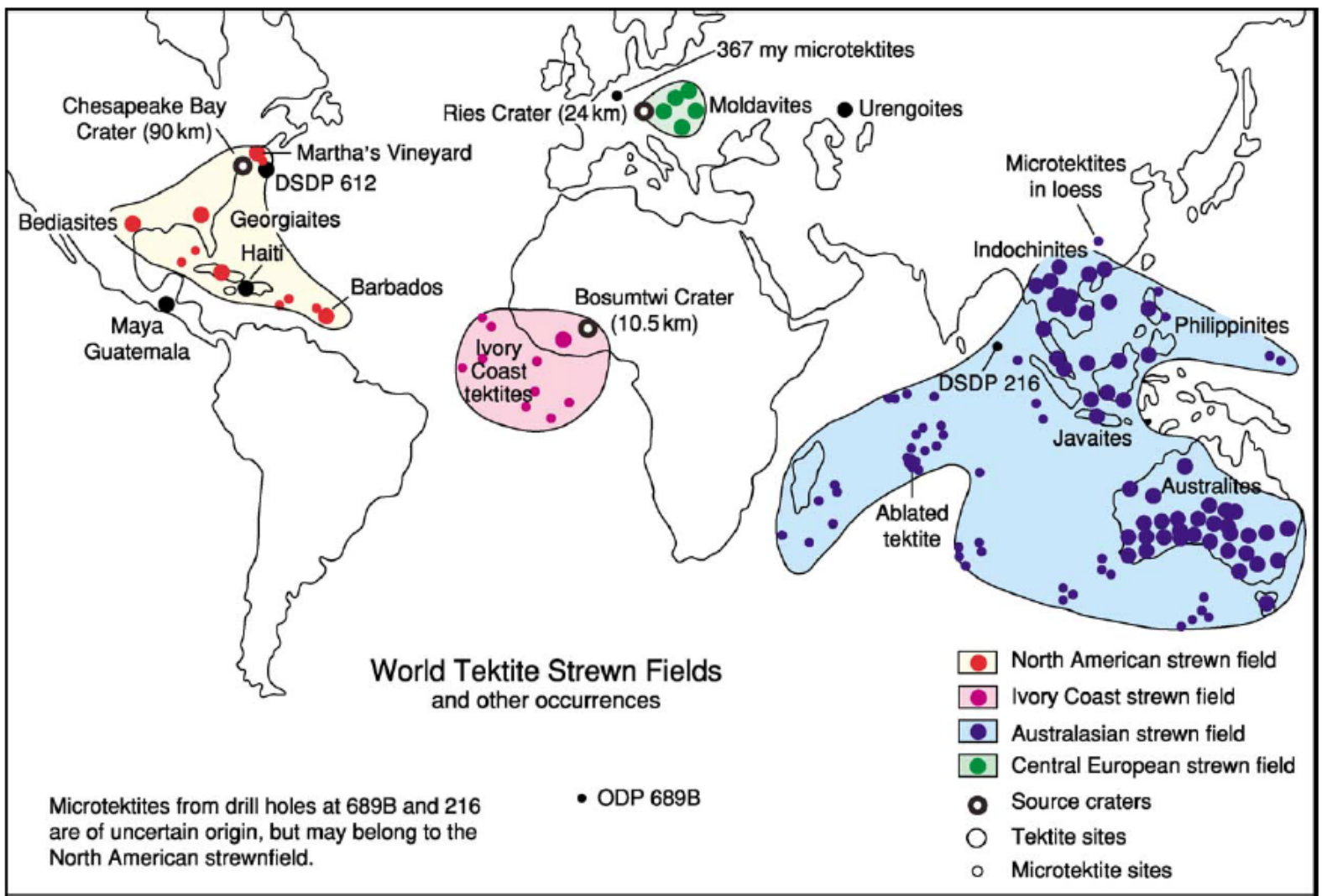

Figure 1.2: Known tektite strewn fields and finds, from McCall (2005). 


\subsection{3: Strewn Fields}

Tektites are found in discrete clusters across the Earth's surface, known as strewn fields (Figure 1.2). Tektites are still rare, geologically, and can be difficult to find (Faul, 1966). Each field corresponds to a distinct impact event, much like the area immediately surrounding a recent meteorite fall. These fields can comprise an extensive surface area, however, and large numbers of tektites exist within each. There are only four tektite strewn fields that are recognized. Some minor distributions of tektite-like material do exist, and may be classed as minor strewn fields (O'Keefe, 1976). Known generally by their geographic locations, the four strewn fields are the Australasian field, the Czechoslovakian field, the North American field and the Ivory Coast field. The North American field, at $34 \mathrm{Ma}$, is the oldest. The

Czechoslovakian, Ivory Coast and Australasian fields have K/Ar ages of $15 \mathrm{Ma}, 1.3 \mathrm{Ma}$, and 0.7 Ma, respectively (Gentner et al., 1969). By weight, the North American field contains 1 billion tonnes of glass, while there are 200 million tonnes in the Ivory Coast field and 100 million tonnes in the Australasian field (Glass, 1990). Impact craters have been identified as the potential source of tektites for all but the Australasian field. The Ries impact structure (Germany) and the Bosumtwi crater (Ghana) have been linked to the Czechoslovakian and Ivory Coast fields, respectively. The more recently discovered Chesapeake Bay structure is the likely source crater for the North American field (McCall, 2005). The fields are of varying sizes and their associated tektites are characterized by their age and slight differences in color and chemical composition. The tektites of the largest strewn field, the Australasian field, possess slightly different chemical and physical properties at different locations (Faul, 1966). Splash- 

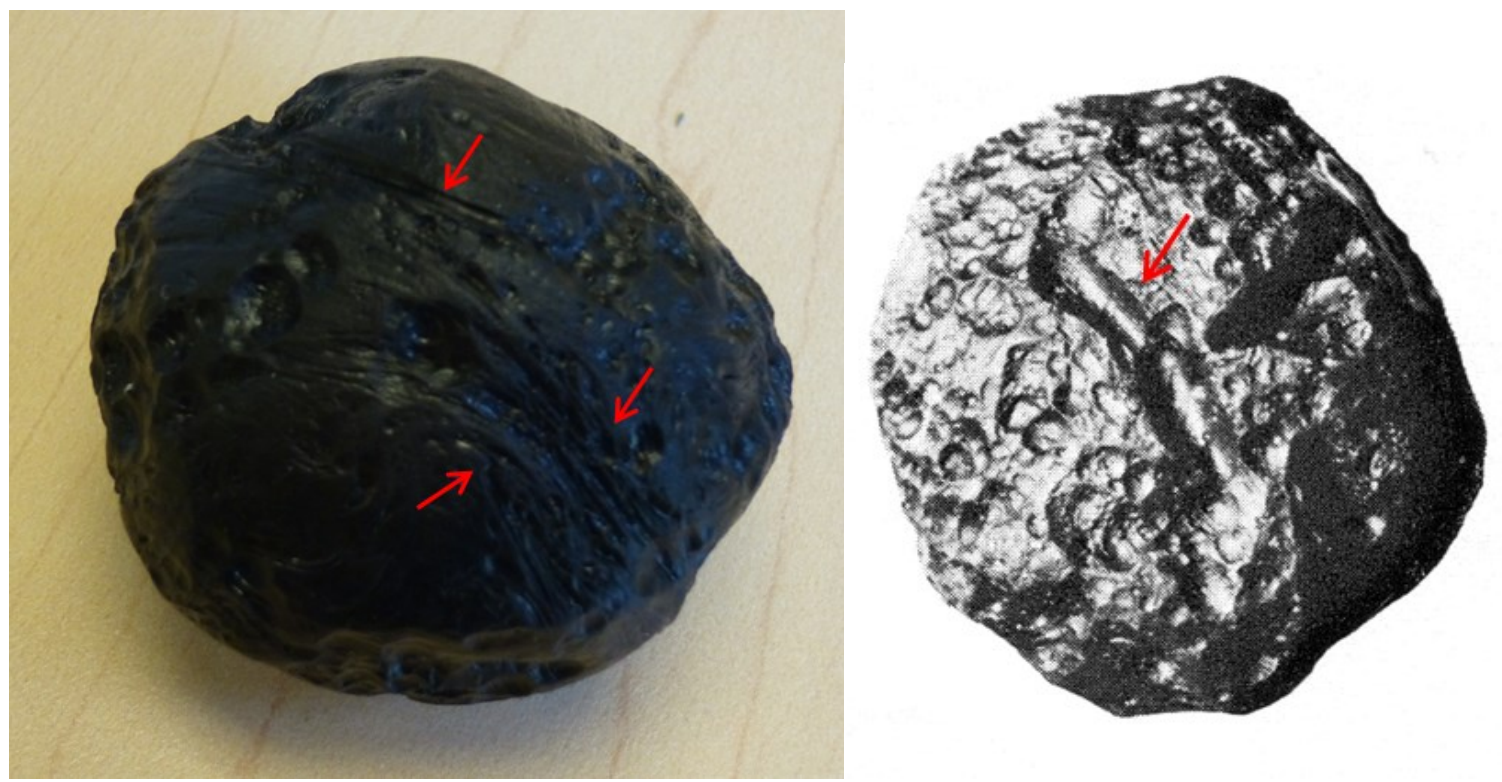

Figure 1.3: Common features on tektites. Left: irregular schlieren, the result of melt flow on tektite TO2. Sample diameter is approximately $4 \mathrm{~cm}$. Right: Meandrine grooves, from O'Keefe (1976). Size of sample is unknown. 
form tektites are found in all four of the strewn fields. Muong Nong and ablated tektites are found solely in southeast Asia and Australia, respectively (Glass, 1990).

Tektites are often found on the ground surface or in much younger sediments, usually gravels, suggesting that some form of post-fall transport has occurred (Faul, 1966). This has the potential to spread tektites over a wider area than that in which they fell. Microtektites have been found in deep sea sediments and have been associated to the same known fields, except the Czechoslovakian, based on their locations. For example, microtektites found in the Indian Ocean and the Philippine Sea are linked to the Australasian field (Glass, 1974).

\subsection{4: Tektite Exteriors}

Tektites are usually about 0.1 to $20 \mathrm{~cm}$ in size, and a few reach several hundred grams in weight. The Moung Nong tektites are typically larger than the other forms and can exceed these measurements (Stauffer and Butler, 2010). Splash-form samples are most often the size of a walnut (Barnes, 1967). Tektites are usually black in color. Thin edges have a hint of translucency that will change this observation to yellowish brown. Some samples have been known to display a variety of colors, such as brown, green or gray (Barnes, 1967).

The exterior of splash-form tektites are marked by hemispherical pits (sometimes known as cupules), as well as striations and grooves (Figure 1.3). These characteristic features, known as surface sculpture, are formed through a process termed "corrosion" (O'Keefe, 1976). Hypotheses on the origins of these features include aerodynamic ablation or dissolution by ground water (Glass, 1974). Degassing of the tektite may have also been a common process in pit formation (Stauffer and Butler, 2010). O'Keefe $(1967 ; 1976)$ reasons that most of the surface 
sculpture must have happened prior to the tektites arrival on the Earth's surface. He points towards the overall lack of features on broken surfaces as evidence. He believes that common features such as pits and grooves were produced by the aerodynamic effects of turbulent gas flow in the Earth's atmosphere, as the tektite was falling. Tektite surfaces can be altered by ablation, resulting in a bald spot. This area is free of any corrosive features previously discussed, such as hemispherical pits. Striae, known as schlieren, often stand out in relief, or form streaks, on the tektite surface. These thin folded structures continue through the interior of the sample, and represent different glass compositions (O'Keefe, 1976; Glass, 1990). They are not seen on all samples, and their appearance can vary, from appearing discretely to quite noticeably. Melt flow, characterized by rolled up flanges and wavy flow ridges can also be found on the surface of some tektites. The irregularity (wavy and wandering) of the schlieren may also be partly due to melt flow. Hemicylindrical features known as meandrine grooves can be found on the surface of some tektites. The grooves, postulated to indicate the anterior surface of the tektite in flight, are interpreted as thermal cracks that have been enlarged (O'Keefe, 1976).

\subsection{5: Tektite Interiors and Chemistry}

The interior of tektites are most often homogenous. They have air pockets, known as vacuoles, and small inclusions, amounting to approximately $0.1 \%$ of the total volume (O'Keefe, 1976). Common

internal features are vacuoles, fingers, flow structure, and layering while less common features include mineral inclusions, metallic spherules and rayed bubbles (Barnes, 1967). Vacuoles can be found throughout the tektite body and, in some cases, are associated with glass inclusions. 
Fingers are light colored inward extensions within the tektite body. Richer in silica than the surrounding glass, fingers are a few millimeters in length and usually end in pits on the surface (Barnes, 1967). They are not to be mistaken for the corrosive pits that are also present. The layers of schlieren observed on the surface continue through the interior and can be identified by their varying refractive indices. Like the interior, the schlieren are often contorted as a result of the tektite material flowing over the surface while molten (Glass, 1990). The most common inclusion is a form of silica glass known as lechatelierite that is likely the result of the melting of quartz grains. Lechatelierite, common in impact glasses, is not present in obsidian (Glass, 1990; Barnes, 1967). Minerals such as quartz, coesite, zircon and nickel-iron spherules (mainly kamacite, $<0.5 \mathrm{~mm}$ in size) have been found in some tektites (O'Keefe, 1976; Barnes, 1967).

Tektites are predominantly composed of silica, with $\mathrm{SiO}_{2}$ in excess of 65 wt.\% (Glass, 1990). They can be distinguished from volcanic glasses by their low water content $(<0.02 \mathrm{wt} . \%)$, the oxidation state of iron (mostly $\mathrm{Fe}^{2+}$ ) and higher ratios of $\mathrm{MgO} / \mathrm{SiO}_{2}$ and $\mathrm{K}_{2} \mathrm{O} / \mathrm{Na}_{2} \mathrm{O}$ (Glass, 1990).Trace elements found in tektites include, but are not limited to, barium, cobalt, lead, lithium, nickel, the lanthanide group, thorium and uranium (Barnes, 1967). Sandstones, namely greywackes and arkoses, are very similar in major and trace element quantities, and possess comparable inter-element variations (Taylor and Kaye, 1969). The chemical composition of tektites varies based on the location where they were found. Tektites from different fields can be distinguished by their major oxide abundances, namely variations in $\mathrm{SiO}_{2}, \mathrm{MgO}, \mathrm{CaO}$ and $\mathrm{K}_{2} \mathrm{O}$ (Koeberl, 1990). 


\section{3: Physical properties of meteorites and tektites}

\subsection{1: Introduction to Physical Properties}

The study of physical properties of geological materials is a broad subject that is useful in many fields and on a number of scales. Some examples include density, magnetic properties, and elastic properties (e.g. P- and S-wave velocity, Poisson's ratio), to name a few. The measurement of physical properties has been extended from terrestrial material to astromaterial, such as meteorites, for density (Consolmagno and Britt, 1998; Smith et al., 2006b; Macke et al., 2010), magnetic properties (bulk susceptibility, frequency dependence, degree and shape of anisotropy) (Rochette et al., 2003; Smith et al., 2006a), porosity (Consolmagno et al., 1998; Wilkison et al., 2003; Fry et al., 2013a; Ralchenko, 2013), and thermal conductivity (Consolmagno et al., 2013).

Astromaterials such as meteorites are valuable resources for researchers interested in studying our solar system. They are considerably older than the Earth and, in some cases, represent material we would not be able to access otherwise. The importance and rarity of this material has spurred researchers to develop specialized laboratories and procedures to preserve and protect the material. While destructive methods have been employed often in the past, non-destructive methods are now favoured to diminish the risk to samples. In the case of meteorite falls (e.g. Sikhote-Alin, Kavarpura), minimizing any changes is essential because such samples have not yet had a chance to be affected by weathering, and are the best window to researching other bodies in the solar system. 


\subsection{2: Measuring Density}

Density $(\rho)$ is the quotient of a mass, $m$, and a volume, $V$, for any given material (Schön, 2011). In rocks, density depends on the mineral composition and porosity of the sample. Void spaces or cracks in a sample are referred to as porosity. This leads to two definitions of density in the nomenclature; bulk density, which is based on a sample as a whole, and grain density, which measures only the solid matter in the sample and ignores pore space (Consolmagno et al., 2008). A secondary method to express density, specific gravity, exists. It is the ratio of the density of the sample to the density of water, or reference liquid, at a specified temperature. Many older studies express results in terms of specific gravity. In laboratory conditions, there is little variation in ambient temperatures and, in practice, specific gravity and density are the same (Carmichael, 1989).

The difficulty in calculating the density of a meteorite or tektite sample lies in measuring its volume. The irregularly shaped samples can often pose a challenge. The time honoured Archimedean method is to submerge the sample in a fluid and to measure the volume of the displaced fluid. Weighing the sample when suspended in water is a variation of this method. Specific gravity can be determined by calculating the difference in weight between the two media. The issue with using these methods is that they are invasive and potentially destructive. Immersing a sample in fluid can drastically affect any future studies and results. Meteorites are not in chemical equilibrium with the Earth's atmosphere and, as a result, can be very sensitive to the effects of weathering (Kohout et al., 2008). Exposure to an oxidizing agent, such as water, can cause chemical alteration (and thus mineralogical changes) to the sample. In the case of meteorite finds (e.g. Canyon Diablo) that an estimated 49,000 of years of exposure has 
already occurred; however, it still remains desirable to minimize any changes introduced by the researcher. Fluids can also complicate density studies by leaving a residue on or, depending on permeability, in the sample. Fluid residue can decrease the reliability of subsequent results (Consolmagno et al., 2008).

Chapman et al. (1964) employed a somewhat different method to determine the density of tektites. The lower density of tektites allow for a liquid flotation technique to be used. Ordinary tap water was modified by titrating a zinc iodide solution to incrementally increase the liquids density. The tektite was immersed in this solution, while the concentration of the $\mathrm{ZnI}_{2}$ solution was incrementally increased, until the sample floats. At this point, specific gravity was recorded.

For meteorites, an alternate method is to cut the sample into easily measured shapes such as cubes, or slabs. An unknown amount of stressing or cracking of the surface, however, may create new pore spaces during sample preparation (Britt and Consolmagno, 2003). Some researchers, in an attempt to be less destructive to the sample, pack the sample into clay instead. The clay can be molded into a measurable shape to produce a volume. The meteorite is then removed, and the clay compressed and remolded. The difference in volume between the two clay shapes is the volume of the meteorite. Difficulties arise due to inconsistent packing of the clay or possible contamination of the sample (Consolmagno et al., 2008). Overall, these methods produce less reliable results than desired; eg. They can be destructive, and their accuracy has the potential to be low.

In lieu of using the methods described above, Consolmagno and Britt (1998) introduced the use of microscopic glass beads to simulate an Archimedean fluid. The beads are chemically 
unreactive and while they can follow the shape of the sample, they will not enter its interior. In short, once the operator is adept with the technique, the bead method provides a quick, cheap and reliable method for measuring bulk volume non-invasively and non-destructively. The procedure and its limits are fully described in section 2.2.

Smith et al. (2006b) introduced 3D laser imaging, the most recent method to measure bulk volume. As the name implies, a laser is used to create three dimensional images of the sample surface that can be combined to create a detailed and volumetrically accurate 3D computer model. The principles and procedure of this method are fully described in Section 2.1. The technique is easily scalable for a range of sizes, and has produced results that are consistent with other methods (Smith et al. 2006b; McCausland et al., 2011; Fry et al., 2013a). There is a significant time commitment with this method, at least 2-3 hours, to achieve a single measurement. This makes its use somewhat impractical for large numbers of samples.

\subsection{3: Meteorite Densities in the Literature}

There are many studies that focus on the density of meteorites in the literature, covering hundreds of samples. The majority of publications focus on stony meteorites, with little mention of iron meteorites, if any (e.g. Flynn et al., 1999; Britt and Consolmagno, 2003; Wilkison et al., 2003).

Farrington (1907) reports the densities of dozens of iron meteorite fragments. This monograph merely collects and reports the work of other researchers. The volume records the original source of the density measurements, some of which date as far back as the year 1800 . It does not include an account of how the results were achieved, but it is reasonable to assume 
that it was some form of fluid measurement. While the oldest resource found, it also contains the largest and most diverse sampling of iron meteorite densities. The Field Museum of Natural History also published other, later monographs on meteorites as part of its geological series. While not dedicated to density, they do include it as part of their meteorite descriptions, in the form of specific gravity (e.g. Roy and Wyant, 1949; 1950).

Henderson and Perry (1954) conducted a review and note that a frequent error in iron meteorite density measurements is the use of oxidized samples. After providing examples of how a small amount of oxide results in a noticeable density difference, they also note that many measurements must be in error from oxidization alteration. They carefully selected and prepared (as thin slices) the samples in their study so that they were free of any oxides or low density inclusions. This allowed them to find the bulk density of only the iron-nickel portion of the meteorite. They examined how density changed with heating and mineral content. A large portion of this publication was the comparison between measured density and density calculated from meteorite composition. The comparisons at times used data that had previously been published by other researchers.

Consolmagno and Britt (1998) included several iron meteorite density measurements as part of their landmark paper introducing the Archimedean bead method. McCausland et al. (2011) report the density of a single iron fragment. Other references to iron meteorite densities come as a mere mention and are not a major part of the author's research. Usually a number is given, but the publication lacks a description of how that figure was obtained (Vdovykin, 1973; Lang and Franaszczuk, 1989). 


\subsection{4: Tektite Densities in the Literature}

As in the case of iron meteorites, information on the density of tektites is fairly scarce. In many instances, a number is given, but there is no mention of how the measurement was made. There are few studies on tektite density; they mostly focus on the Australasian field. Baker and Forster (1943) carried out a statistical examination of tektite density. They found a relationship between shape and density, and none between weight and density. In areas of where large numbers of samples are available, teardrop tektites are listed to have the highest density while lenses and ovals (perhaps discs and ellipsoids) have the lowest densities. They also noted that density varies with location and could indicate a potential direction of flight for the tektites across the Australian continent. Perhaps the most comprehensive study was the landmark publication by Chapman et al. (1964). They created a histogram of density population after examining 6000 tektites, and found a series of partially overlapping density clusters within the Australasian field. Notably, the densities at opposite ends of the Australasian field are indistinguishable from one another, while there are sizable variations among clusters that are closer together. The implication is that the tektites are more closely related to relatively distant clusters than to their closer neighbors, for the same event. The most recent study of tektite density was done by Schmude (2002). He found that density and tektite shape were not correlated, in contradiction of the findings of Baker and Forster (1943).

\section{4: Research Objectives and Thesis Structure}

3D laser imaging is a relatively new technique in the earth sciences. The first reported successful use of this technique was Herd et al. (2003). In the laboratory, it has been so far 
used to measure the density of a wide variety of stony meteorites - including frozen fragments of the Tagish Lake carbonaceous chondrite kept in a cold room (Ralchenko, 2013) - and to map fractures on drill cores (Olson, 2013). The detailed 3D models are kept as archives for further studies.

The research project presented in this thesis aimed at extending the applicability of 3D laser imaging to other geomaterials. Its overarching goal was to test the feasibility of using 3D laser imaging for capturing fine surficial details and measuring the density of iron meteorites and tektites. A contribution of the thesis was to streamline any challenging imaging issues, and to mitigate any errors that arose from modeling these two materials. Comparison with the more established Archimedean method provided an opportunity to validate the laser-derived density measurements. McCausland et al. (2011) and Fry et al. (2013) have shown that the two methods produce similar results (1.90\% and $1.07 \%$ average difference, respectively) when investigating stony meteorites.

Iron meteorite density data are under-represented in the literature, with no extensive study having been conducted in decades. The same could be said for tektites. This study contributes approximately 41iron meteorite and 10 tektite density.

3D laser imaging has been used only once in the past to measure the density of an iron meteorite sample - a $56.94 \mathrm{~g}$ fragment of Mundrabilla (McCausland et al., 2011) - and the results were less than satisfactory. Image alignment and subsequent model building was difficult due to its unusual Y-shape. Its small size was on the limit of applicability of the Archimedean bead method. This led to a large difference (6.5\%) between the densities measured by the two methods (McCausland et al., 2011). This study covers 41 samples, 
including 5 suites of samples (more than 4 samples that have come from the same meteorite). It allows an investigation of the variability between fragments, and searches for trends between density and the various meteorite classes. Iron meteorites presented a new range of features that have not been previously tested by the camera; they have an extensive range of shapes, from brick-like to shrapnel-like, and metallic surfaces. As seen from the Mundrabilla sample, some shapes, especially those lacking salient features, are challenging for image alignment. At the onset of this study, it was unknown how a reflective metallic surface would interact with the laser.

The objectives of creating 3D models of tektites were fairly similar. First, this study aimed to investigate if laser imaging is applicable to this material, given its glassy nature, and if so, to mitigate any challenges that might arise. In the past, imaging obsidian had not produced reliable results. Should it work, the study sought to further demonstrate the capabilities of the instrument by capturing classic tektite surface features, such as the hemispherical pits (cupules) and flow structure (defined by schlieren), and the different splash-form shapes. As in the case of iron meteorites, laser-derived tektite densities can be calculated. Using the models themselves in a future research direction that involves realistic simulations of airflow over the tektite while inflight is further explored.

Following this introductory chapter, Chapter 2 describes in more detail the Archimedean bead and 3D laser imaging methods. It explains the theory behind each method, along with their respective merits and potential errors that may occur. Chapter 2 details the steps taken from start to finish to complete the measurements. Chapter 3 provides new density data for 
the iron meteorites, using the Archimedean bead method and 3D laser imaging, and investigates their potential impact. It discusses any issues that arose using either method, and how they can be mitigated. Finally, chapter 3 compares the results from this study with those found in the literature. Chapter 4 focuses on the portion of the study involving tektites, and follows much of the same layout as chapter 3. Chapter 4 introduces a potential use of the 3D models in aerodynamic flow simulations. Finally, chapter 5 reviews the lessons learned during this research project and in what direction future studies could proceed. 


\section{Methods}

\section{1: Laser Imaging}

\subsection{1: Principle}

The VIVID 9i non-contact 3D digitizer uses the light strip method to illuminate an object with a laser beam. This beam is projected through a cylindrical emitting lens, where it becomes a strip of light that is steered by a galvano mirror, to transversely scan the target object from top to bottom (Figure 2.1). The diffusely reflected light is captured by the receiving lens, focusing it on a charge-coupled device (CCD). The camera uses optical triangulation of the projected and reflected raypaths to convert the CCD output into Cartesian coordinates, producing a 3D image with a resolution of $640 \times 480$ voxels. A voxel is simply the $3 D$ analogue of pixels in a $2-D$ photograph. It is composed of three X, Y, Z Cartesian coordinates, with Z corresponding to depth of the image, in addition to the camera-target distance. Intensity information is not recorded by the VIVID 9i camera. The image is saved and exported as an ASCII point cloud. As well, the camera will capture a color photograph of the object while the laser beam is not being emitted. The laser is unable to capture regions that are not directly in the line of sight. Crevices, overhangs and the bottoms of deep pits are the most common occluded features.

The 3D images acquired with the camera will always have a resolution of $640 \times 480$ voxels. As a rule of thumb, the closer the object is to the VIVID 9i, the more detailed the image will be since the pint cloud will be less dispersed. The volume of around the object measured by the camera will in turn decrease because the field of view of the camera will be limited by the closer focus. The camera comes with 3 different receiving lens for image capture; wide lens, middle lens and tele lens. The tele lens is best suited for imaging small objects, such as 
meteorites, and was used the most. The other two lens can image successively larger objects, but at lower accuracy. The images, and thus final models produced will not be as detailed as with the tele lens, because the ASCII point cloud produced will have a lower concentration (expressed in number of voxels per unit area on the surface of the target object) than with the tele lens.

Table 2.1 lists the input ranges of the VIVID 9i at distances of $0.6 \mathrm{~m}$ and $1 \mathrm{~m}$ (Konica Minolta, 2006). The $X, Y, Z$ input ranges corresponds to the volume captured by the camera at a set distance. The maximum possible camera-target distance is $2.5 \mathrm{~m}$, when the camera is set to extended mode. The input range also allows for the concentration of voxels to be quickly calculated. The VIVID 9i records $640 \times 480$ (307200) voxels at most per image. At a range of $70 \mathrm{~cm}$, using the tele lens, the image will capture an area of $\sim 12580.9 \mathrm{~mm}^{2}(129.7 \mathrm{~mm}$ [x-axis] $\mathrm{x}$ $97 \mathrm{~mm}$ [y-axis]. This means that there is approximately 24.4 voxels $/ \mathrm{mm}^{2}$ when a flat surface is imaged at a normal incidence. The system is ideal for objects under a meter in size, as defined by the $X$ and $Y$ values in Table 2.1 .

\begin{tabular}{|c|c|c|c|c|c|}
\cline { 4 - 6 } \multicolumn{2}{c}{} & \multicolumn{3}{c|}{ Input Range } \\
\hline \multirow{2}{*}{ Lens } & Distance & Accuracy & & & \\
& {$[\mathrm{m}]$} & {$[\mathrm{mm}]$} & $X[\mathrm{~mm}]$ & $Y[\mathrm{~mm}]$ & $Z[\mathrm{~mm}]$ \\
\hline Tele & $0.6 / 1.0$ & $0.05 / 0.10$ & $111 / 185$ & $83 / 139$ & $59 / 189.5$ \\
\hline Middle & $0.6 / 1.0$ & $0.10 / 0.20$ & $198 / 329$ & $148 / 247$ & $94 / 306.5$ \\
\hline Wide & $0.6 / 1.0$ & $0.20 / 0.40$ & $359 / 598$ & $269 / 449$ & $150 / 487$ \\
\hline
\end{tabular}

Table 2.1: Manufacturer's specifications for the VIVID 9i (Konica Minolta, 2006)

A turntable can be used to precisely rotate objects so that different surfaces will face the 
camera. The two pieces of hardware, the laser camera and the turntable, are controlled by a desktop PC, using the program "Polygon Editing Tool” (PET) developed by Konica-Minolta. Camera settings such as laser intensity and the focusing distance are set here. In this study, the settings were manually adjusted, for consistency throughout the imaging process.

\subsection{2: Image Capture Process}

The VIVID 9i non-contact 3D digitizer, mounted on a tripod, was approximately $0.7 \mathrm{~m}$ away from where the meteorite or tektite rested on a turntable (Figure 2.2). The laser camera was tilted about $30^{\circ}$ so that the laser would illuminate the side and top of the fragment and capture as much surface as possible. Having part of the side and top the sample in a single image greatly aids in assembling different images into a high-fidelity model. The focus of the camera was set to the center of the turntable for small samples $(\sim 0.7 \mathrm{~m})$ and a bit closer to the receiving lens in the case of a few larger samples $(\sim 0.65 \mathrm{~m})$.

Through the use of the turntable, the sample was rotated in $20^{\circ}$ increments, with an image being taken each time. The turntable provides accurate rotation, with minimal physical contact. The sample was imaged in three separate orientations, so that all edges and surfaces could be fully captured. This method usually resulted in 54 3D images being taken of each sample, to create an image library for future use. Not all images need be used, but this library provides a comprehensive coverage of the sample surface from a number of different angles. In general, it provides a degree of redundancy should a scan of the same surface, at a different angle, prove not to be useful. An example of such a problematic image is when part of the surface is in the shadow of a more prominent feature, creating an occlusion. At times, it was necessary to take 


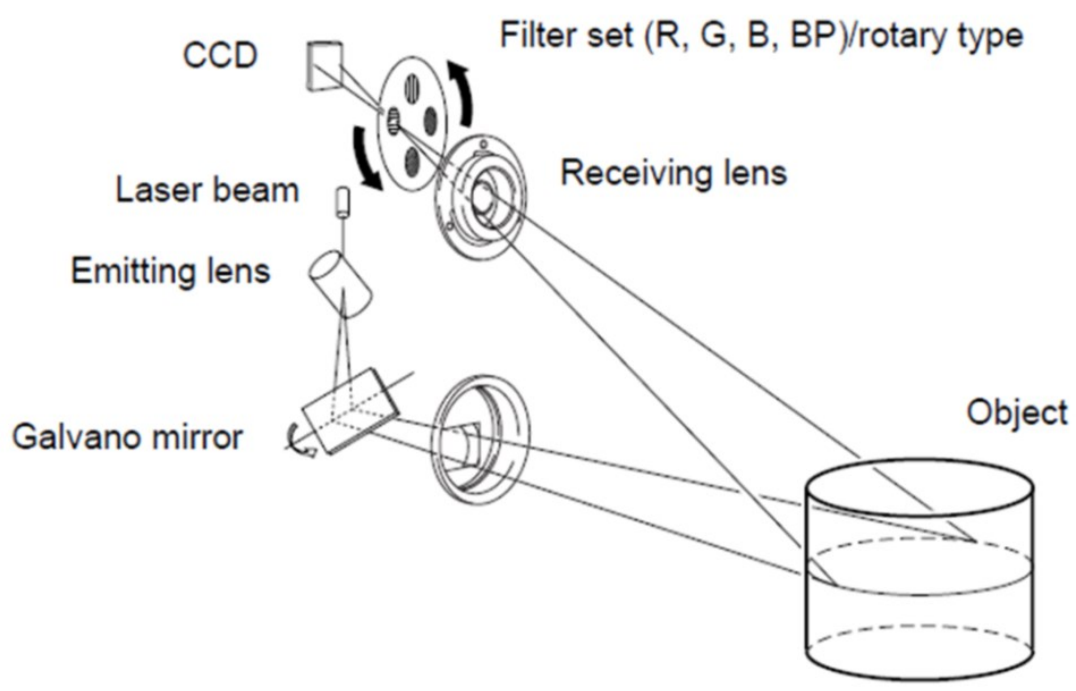

Figure 2.1: Schematic diagram of the VIVID 9i non-contact 3D digitizer operating principle. The emitted light stripe passes through a band pass (BP) filter. A color photograph is obtained through the use of a red-green-blue (RGB) filter. The reflected light illuminates a charge-coupled device (CCD) and the digitizer converts this signal into Cartesian coordinates (Konica Minolta, 2006).

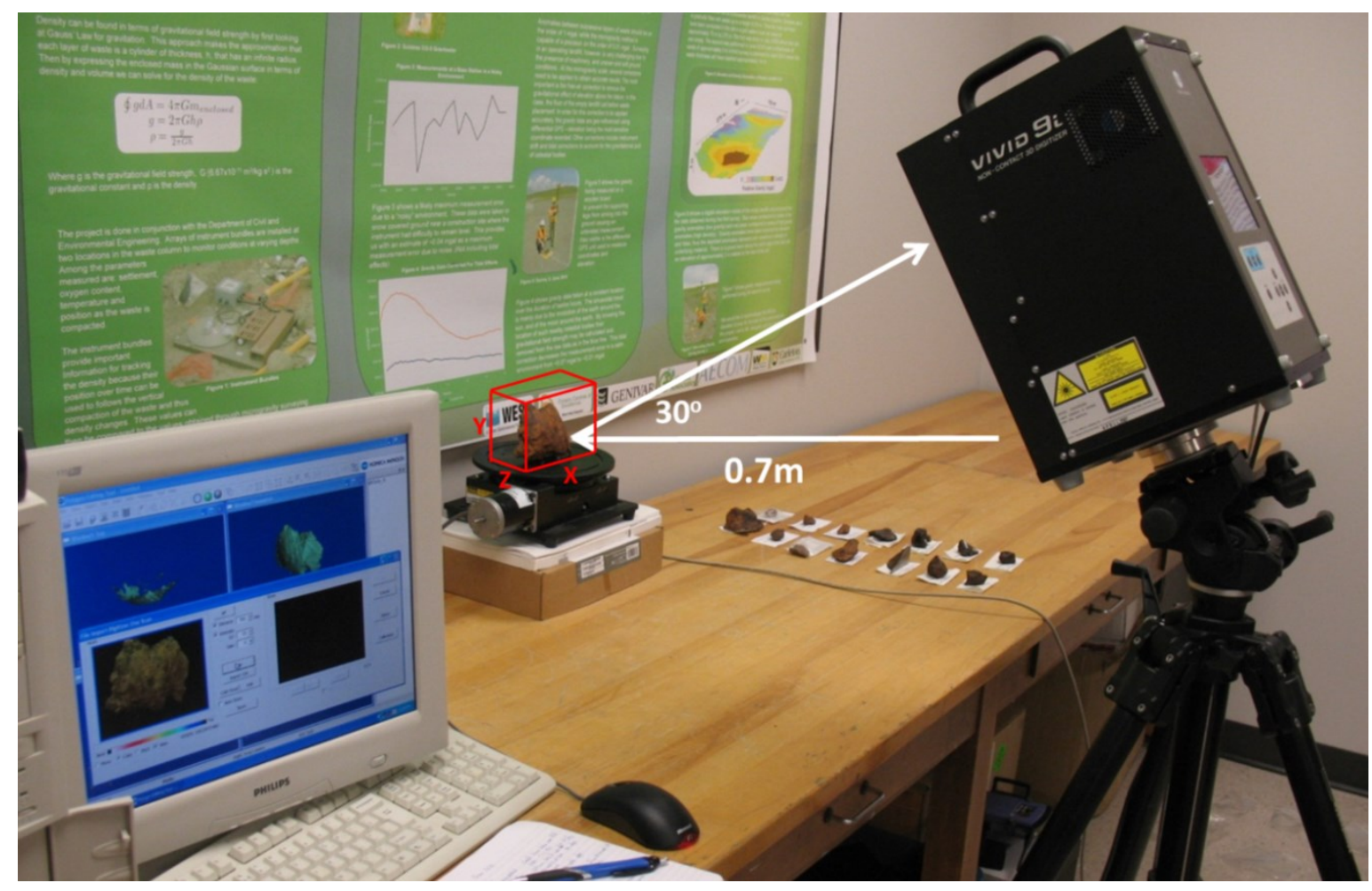

Figure 2.2: A fragment of the Whitecourt meteorite rests approximately $0.7 \mathrm{~m}$ from the VIVID 9i laser camera. The red box indicates the volume scanned by the camera; $x=130 \mathrm{~mm}, y=97 \mathrm{~mm}, z=91 \mathrm{~mm}$. The images can be viewed on the associated computer using PET. An assortment of other iron meteorites is also shown. 
additional images of the sample, to provide extra detail of subtle features that may have been missed originally. This could be because the surface was complicated, with a number of edges and crevasses, or if there was a sharp angle between two sides, such as in the case of cut faces. In the case of Sikhote-Alin meteorite fragment T539, there was a cavity caused by a large regmaglypt on the side; several additional images were required to adequately capture this feature.

The data gathered using the laser camera was stored as an ASCII point cloud (Figure 2.3,2.4A), that was then imported into the Polyworks visualization software tool, developed by Innovmetric Inc. of Quebec City, Canada. Here, the individual images can be assembled by a human operator into a closed 3D model, following the method described in Smith et al. (2006b) and McCausland et al. (2011). An average of approximately 15 of the images will be used, though this number depends on the operator. The Polyworks software fits a mesh of triangles to the surface outlined by the ASCII point cloud. The point cloud is discarded by the program, in favor of the easier to manipulate, less data-intensive mesh (Figure 2.4B). Polyworks allows for separate meshes to be aligned together, by selecting pairs of similar points and allowing the software to lock them together (Figure $2.4 \mathrm{C}$ ). The similar points that are used are usually salient features such as corners, regmaglypts, labels, or anything that is distinguishable in both images.

Three main types of artifacts can be seen in the images (Figure 2.5). The most common artifact is distortion along the image edges due to field of view curvature. Also common is a 'stretched' texture from imaging a surface at a grazing incidence. This causes points to be more dispersed over a surface and little surficial detail to be recorded. This is also the easiest to recognize because the mesh will appear stretched and lack textures similar to the rest of the 

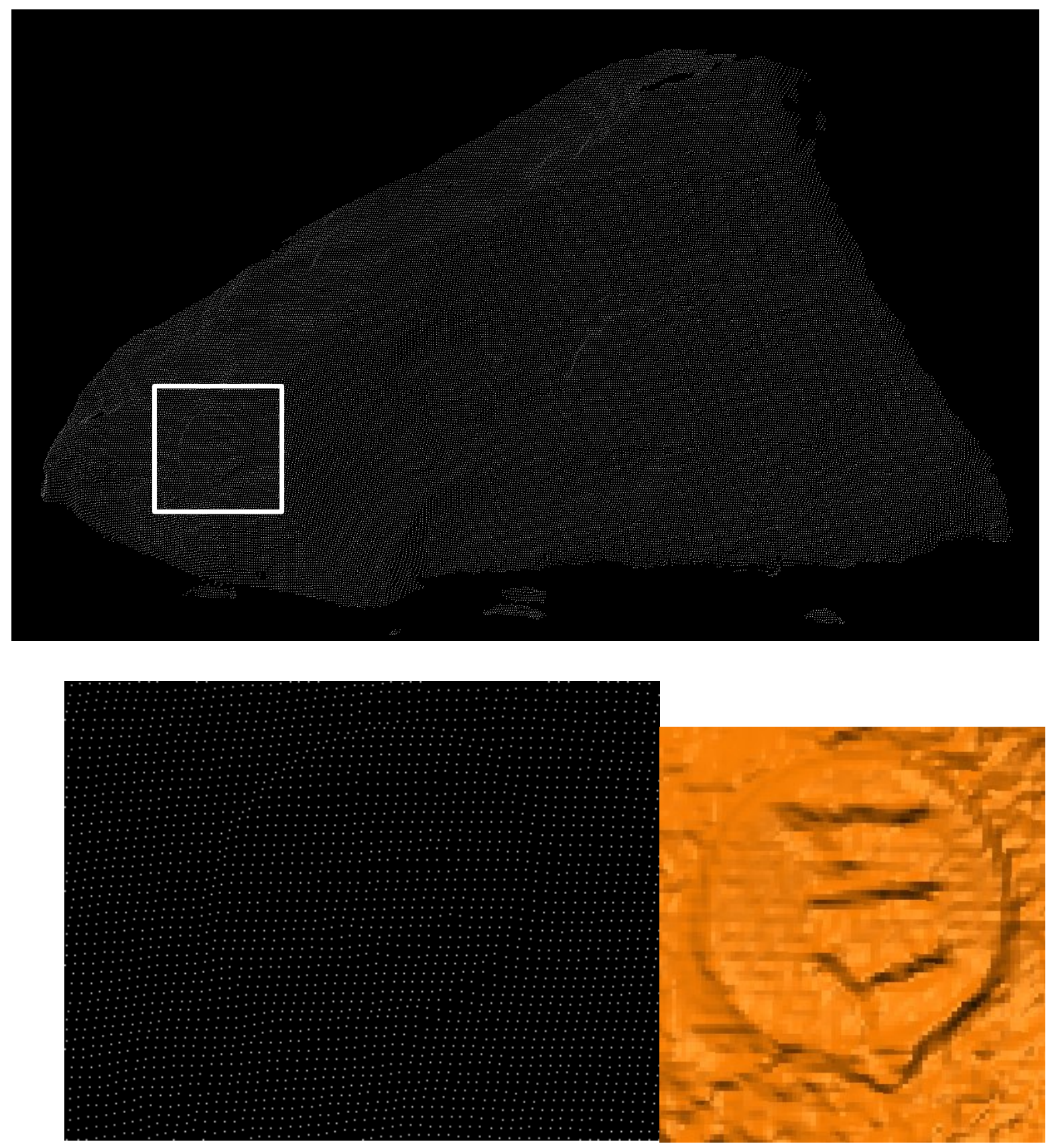

Figure 2.3: Raw data from the laser camera was in the form of a point cloud. The upper image shows the entire point cloud for a single scan. The lower image is an enlarged section $(1 \mathrm{~cm} \times 1 \mathrm{~cm}$ (left) and the corresponding final mesh (right). 


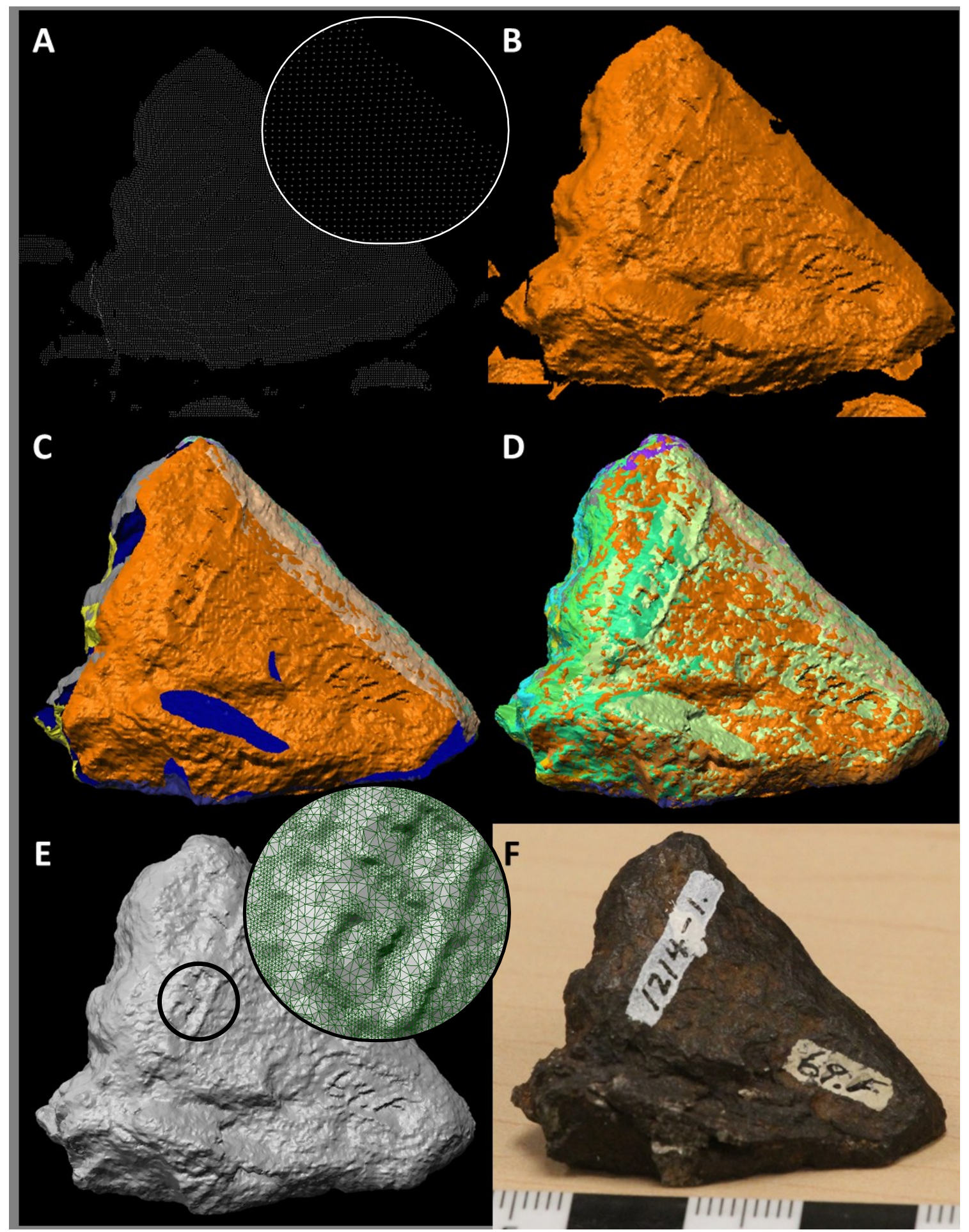

Figure 2.4: A; The point cloud of a single image of a 59.0g fragment of the Toluca (\#1214.001) IAB meteorite (a zoom in area is shown in the circle); B: The mesh that has been fitted to the point cloud; C: Several meshes have been edited and aligned together. Each mesh is a different color, with dark blue corresponding to holes in the image.; D: Surface of meteorite has been completely covered by the aligned meshes. E: The completed 3D model. Excess overlapping mesh has been removed and natural and artificial surface textures can be seen (a zoom in area is shown in the circle); F: A conventional photograph of the fragment, with centimeter scale bar. 
surface. Finally, there may sometimes be spurious protrusions, either as spikes or pits on the surface. During the imaging process, noise may occur, denoted by points that are out of place in the point cloud, usually hovering in front of the surface defined by all the other points.

Polyworks fills in holes during the point cloud-to-mesh computation, and seeks to use these outlying points. The result of using the scattered points is that they cause spurious protrusions or intrusions. Usually these are noticeably different from the rest of the image surface, and easy to remove. Polyworks ignores the misplaced points when they are further away from the point cloud (on the scale of centimeters). This can result in a hole in the mesh, or the presence of the stretched texture, depending on the number of points missing. The best way to prevent this form of artifact is to change the import settings in Polyworks during the point cloud-tomesh computation, so that holes are not filled. By changing the settings, noise is less likely to cause artifacts. Should any protrusions appear, they can be quickly removed by manual editing. An additional artifact, known as "bleeding", can be present. This effect is created by intensity anomalies, or a change in colour of the object, during the imaging process. A strong intensity can overload the CCD detector, with the remainder of the charge spilling over to other parts of the sensor array. A simple check to see if this effect is present can be applied; a white piece of paper with a 2 inch black circle was placed vertically in front of the camera and imaged. Ideally, this circle should not be incorporated into the 3D image, as it is only two dimensional. The circle was quite noticeable in a cursory examination of the 3D image (Figure 2.6), showing that the intensity created by the strong black and white contrast on the page can affect the VIVID 9i. Note that most geomaterials will not display such a strong contrast in intensity and color. 


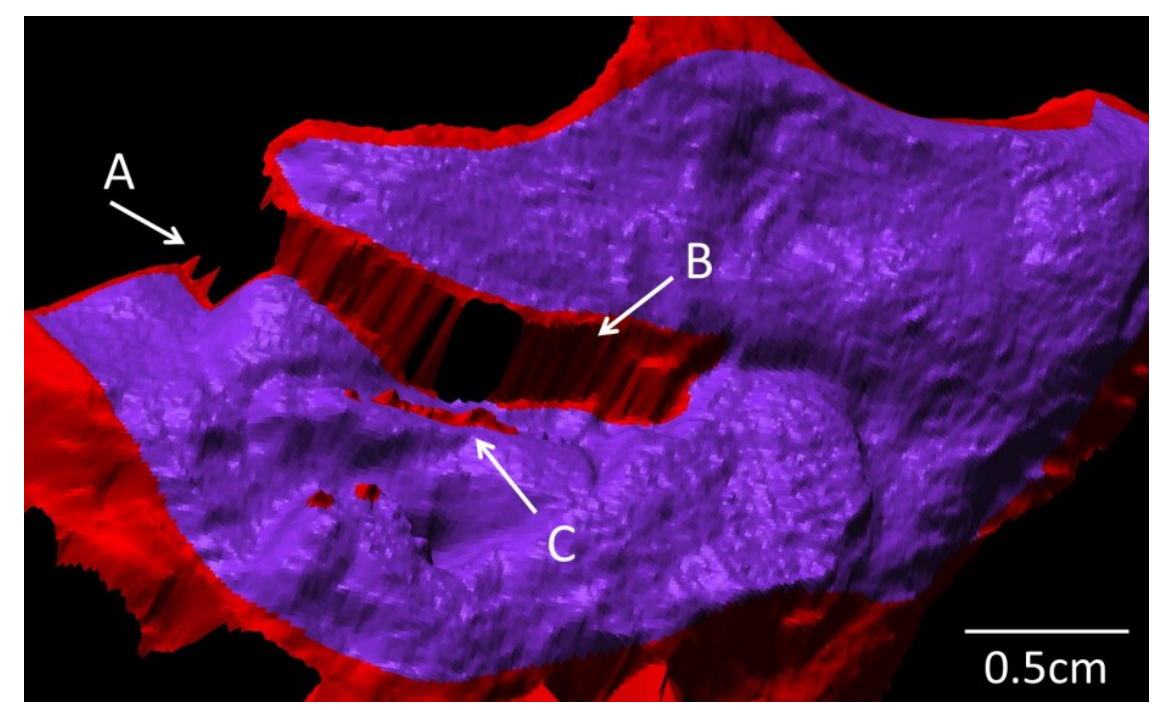

Figure 2.5: Three typical artifacts seen in the mesh image; A: Distortion around image edges; B: Stretched texture from grazing incidence; $C$ : Spurious features from image noise.

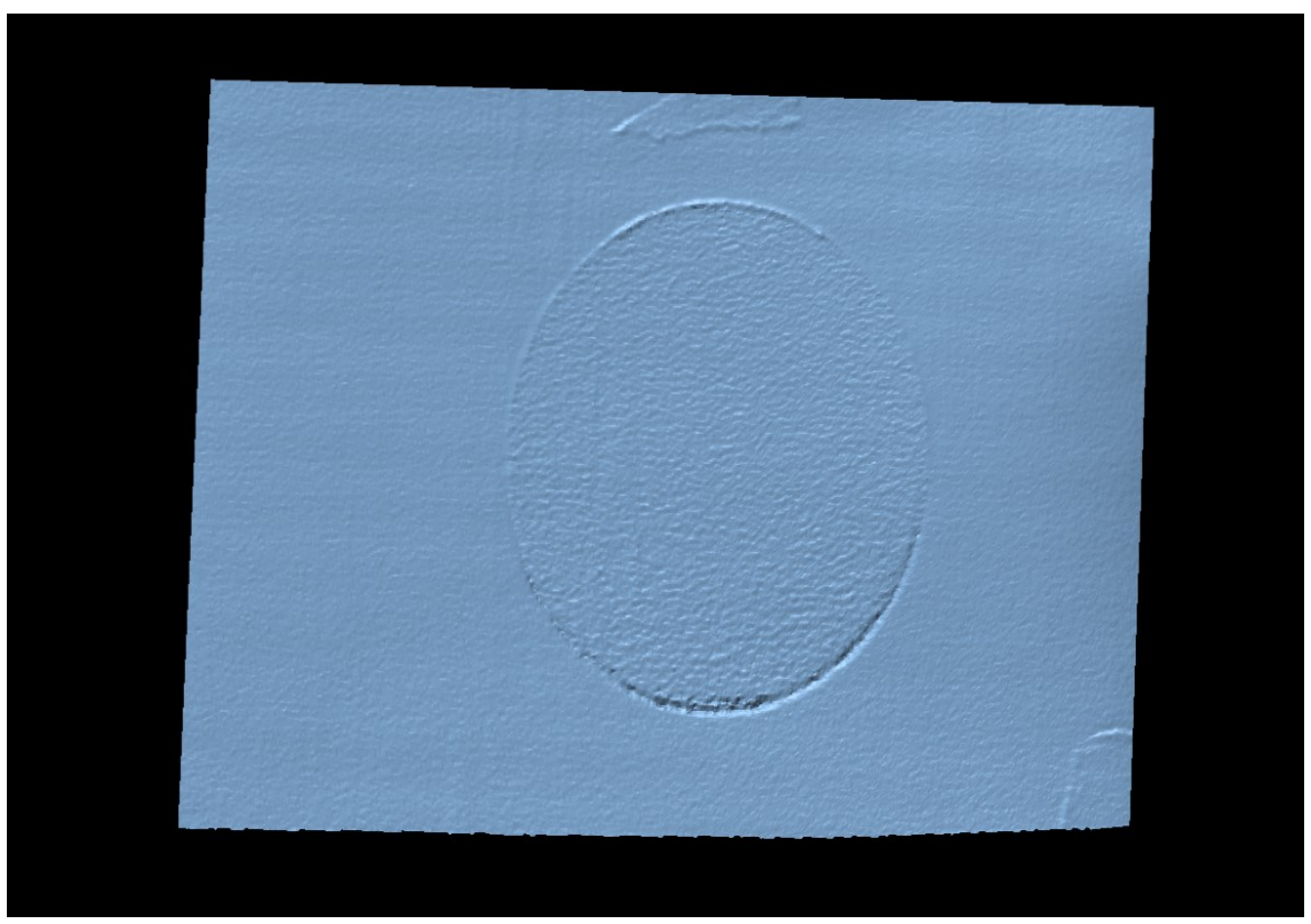

Figure 2.6: 3D Image from the bleeding test. The black 2D circle that has been printed on a white piece of paper is very noticeable. As a comparison, masking tape (which has a physical thickness) has been placed to the top, and side of the circle. 
The best way to negate the effect of artifacts on the final model is to be aware of their existence. The operator can selectively edit an image by knowing how and where an artifact may occur. For example, by routinely editing the edges of each image, the operator can prevent any upturned edges from being incorporated into the final model. In most cases, critically examining each image is enough to prevent artifacts from being incorporated in the final model.

In all cases, the artifacts were easily removed through a simple edit function, where the unwanted parts of the mesh were highlighted and deleted. Removing artifacts before the merge process will result in the final model having a more realistic surface, and can allow for more subtle features to be conserved. Once the model is complete (Figure 2.4D), excess overlap (redundant data) between the meshes is removed and the meshes are merged into a single 3D polygonal model (Figure 2.4E). The polygonal model is then inspected for holes or in some cases, features that may not be true to the physical samples. The surface of the polygonal model may have holes of varying sizes, depending on how closely the original images overlap. Most often, they occur when there is a feature, such as a hole or crevasse, that is difficult to capture fully or when the overlap between two neighboring images is limited. The holes can be filled automatically by Polyworks with a curvature matching mesh, creating a finished, "water tight", model. The Polyworks program has a suite of functions that can be applied to the completed model. Of relevance to this project is its ability to calculate the volume and surface area of the model. 


\subsection{3: Noise Analysis}

Determining error in 3D laser imaging is difficult. For other methods, a reference object (usually a sphere or a cube) is often used to make a comparison between the known and measured volumes. Doing this, however, is challenging because the uniform shape of such an object is impractical to model in 3D. Instead, three measurements for error are proposed, based on different operations that take place throughout the modeling process. The first, described below, is for error from the camera itself. Secondly, The Polyworks program calculates a standard deviation between overlapping images. This metric describes how closely the images that are used to create the model overlap. The final way is to calculate the deviation in results between multiple models (see section 3.3.3). This accounts for variation introduced by different operators creating 3D models of the same object.

Measurement noise is inherent to any metrology device. In this case, the $X, Y, Z$ coordinates generated by the laser camera contain an element of measurement error, predominantly in the Z-axis, which is important to quantify.

A V-shaped, matte metal calibration object (Figure 2.7A) was imaged using the VIVID 9i noncontact 3D digitizer. The two flat planes, only one of which is used, are rigidly connected at a $120^{\circ}$ angle. Initially one side of the object was oriented perpendicular to the laser beam (Figure 2.7B). It was rotated on the turntable in $15^{\circ}$ increments to simulate the progressive imaging process that occurs when imaging samples. The maximum angle measured was $60^{\circ}$. An image of a surface at an angle any higher than $60^{\circ}$ is unlikely to be used due to the unrealistic stretched texture resulting from the grazing incidence of the laser beam described above. 
A principle component analysis (PCA) was used to analyze the point cloud data to quantify the amount of noise present. PCA uses a least squares method to calculate a best fitting plane through a selected volume of points (Mah et al., 2013). Deviation from this plane can be considered as measurement error, as the plate itself is a planar object. A MATLAB script analyses a subset, a sphere, of the point cloud using this method (Figure 2.8) (Mah et al., 2013). The standard deviation about the best fit plane can be used to quantify measurement of error. A measurement radius of $15 \mathrm{~mm}$ was selected to conduct the PCA analysis on the 5 image series (normal incidence $\left(0^{\circ}\right), 15^{\circ}, 30^{\circ}, 45^{\circ}, 60^{\circ}$ of rotation) (Figure $2.7 \mathrm{~B}$; only normal incidence, $15^{\circ}, 30^{\circ}$ shown). Three standard deviations represent $99.7 \%$ of the point population and was plotted on the left-hand vertical axis of Figure 2.9. For all incidence angles, the standard deviation was low, with the maximum being $0.018 \mathrm{~mm}$. This metric provides an error threshold of approximately $0.054 \mathrm{~mm}(\approx 0.018 \mathrm{~mm} \times 3)$. The point population varies with a constant radius, because fewer points will cover an area as the angle of incidence increases (see Figure 2.9).

In an alternative approach (Figure 2.10), the radius of the PCA analysis was modified to maintain a relatively constant point population. A population of $13666 \pm 2$ points is used for each angle considered, corresponding to the baseline PCA analysis (Figure 2.9) with 15mm radius at normal incidence. To maintain a consistent point population, the radius is non-linearly increased to $15.57 \mathrm{~mm}$ at $30^{\circ}, 17.03 \mathrm{~mm}$ at $45^{\circ}$, finally reaching $20.39 \mathrm{~mm}$ at $60^{\circ}$. The radius of the PCA actually slightly decreased to $14.86 \mathrm{~mm}$ at $15^{\circ}$. The standard deviation (multiplied by 3 ) is very similar, varying only slightly from Figure 2.9. The error threshold remains around $0.054 \mathrm{~mm}$. Imaging the plate at a normal incidence produces the highest error. The plate feels very smooth to the hand, however, all mechanical surfaces can be machined to a specific 

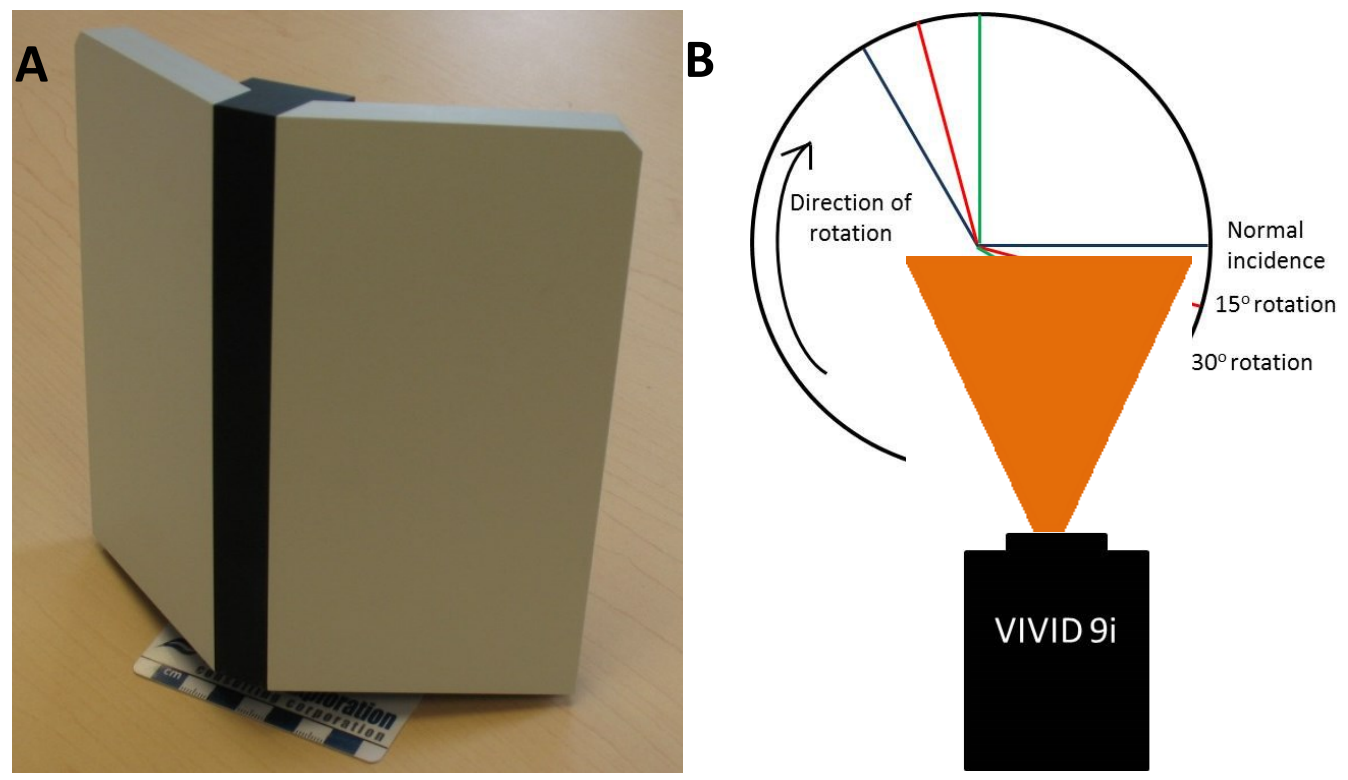

Figure 2.7: A: The metal calibration plate used. The scale card is in centimeters.

B: The positioning of the calibration object as it is rotated during the noise test.

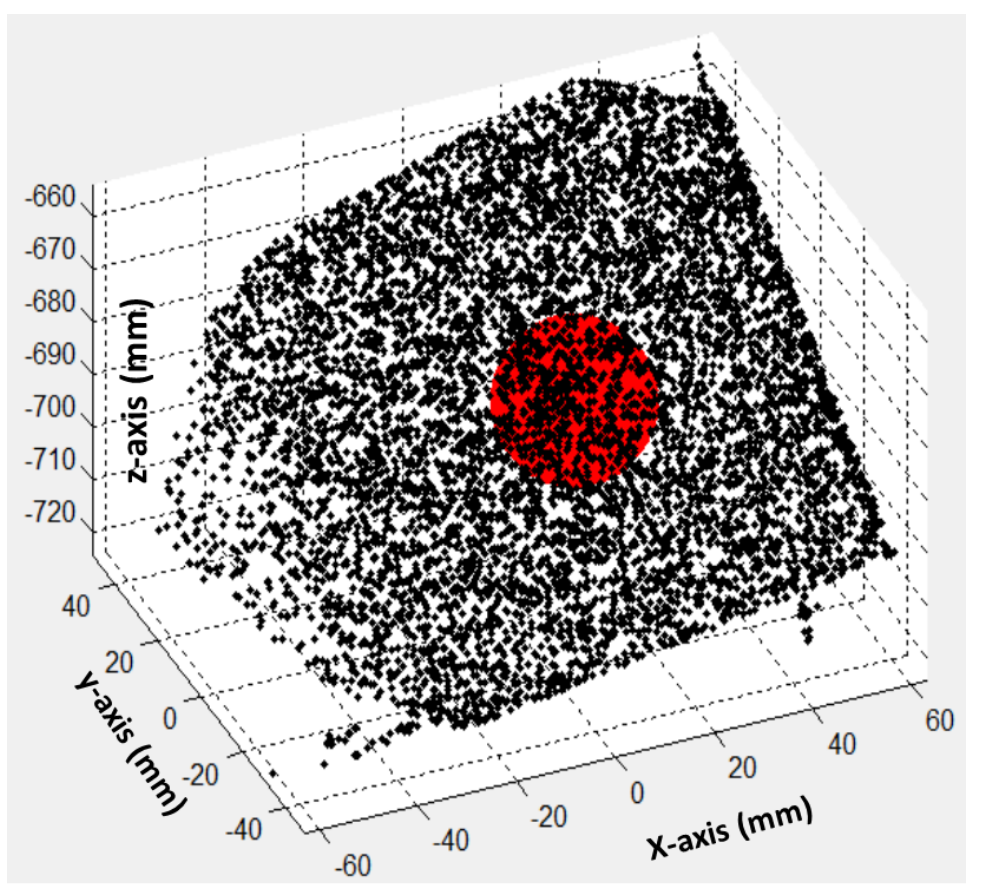

Figure 2.8: A graphical representation from the PCA script developed by Mah et al. (2013) showing the point cloud (202411 points in total) corresponding to a single image of the calibration plate (Figure 2.7A). A red circle (radius of $15 \mathrm{~mm}$, including 13933 points) 
flatness tolerance. The flatness tolerance for the metal calibration plate is unknown, therefore the measurement noise measured using PCA includes the effects related to the flatness of the target. This is a possible reason why the error is the highest when imaging the calibration object at an angle of $0^{\circ}$. Imaging at an angle of $60^{\circ}$ produces the lowest error, perhaps due to a loss of surface texture when imaged obliquely.

The above analyses have shown that the VIVID 9i non-contact 3D digitizer used in this study with the tele lens has a measurement error of $0.054 \mathrm{~mm}$ at $0.7 \mathrm{~m}$ from the target, consistent with the accuracy quoted in the manufacturer's specifications $(0.05 \mathrm{~mm}$ at $0.6 \mathrm{~m})$ (Table 2.1$)$. Some of the tektite or iron meteorite specimens imaged exhibit subtle textures or features smaller than this threshold - schlieren lines or the intergrowth pattern of metallic minerals. These features will be challenging to fully capture.

\section{2: Archimedean bead method}

\subsection{1: Principle}

The Archimedean glass bead method was developed in the late 1990's in response to the need to perform non-destructive bulk volume measurements on meteorites (Consolmagno et al., 2008; Macke et al., 2010). The beads, 100 micrometer silica spherules, are used to simulate the flow of an incompressible fluid. Unlike a liquid, beads will not permeate the interior of the sample and are easily noticed and removed if adhered to the sample's exterior. The glass beads are chemically stable and will not react with the samples being measured.

Following Archimedes' principle, an equal volume of beads will be displaced when a sample is submerged in a container filled with these beads. The density of the beads is known. It 


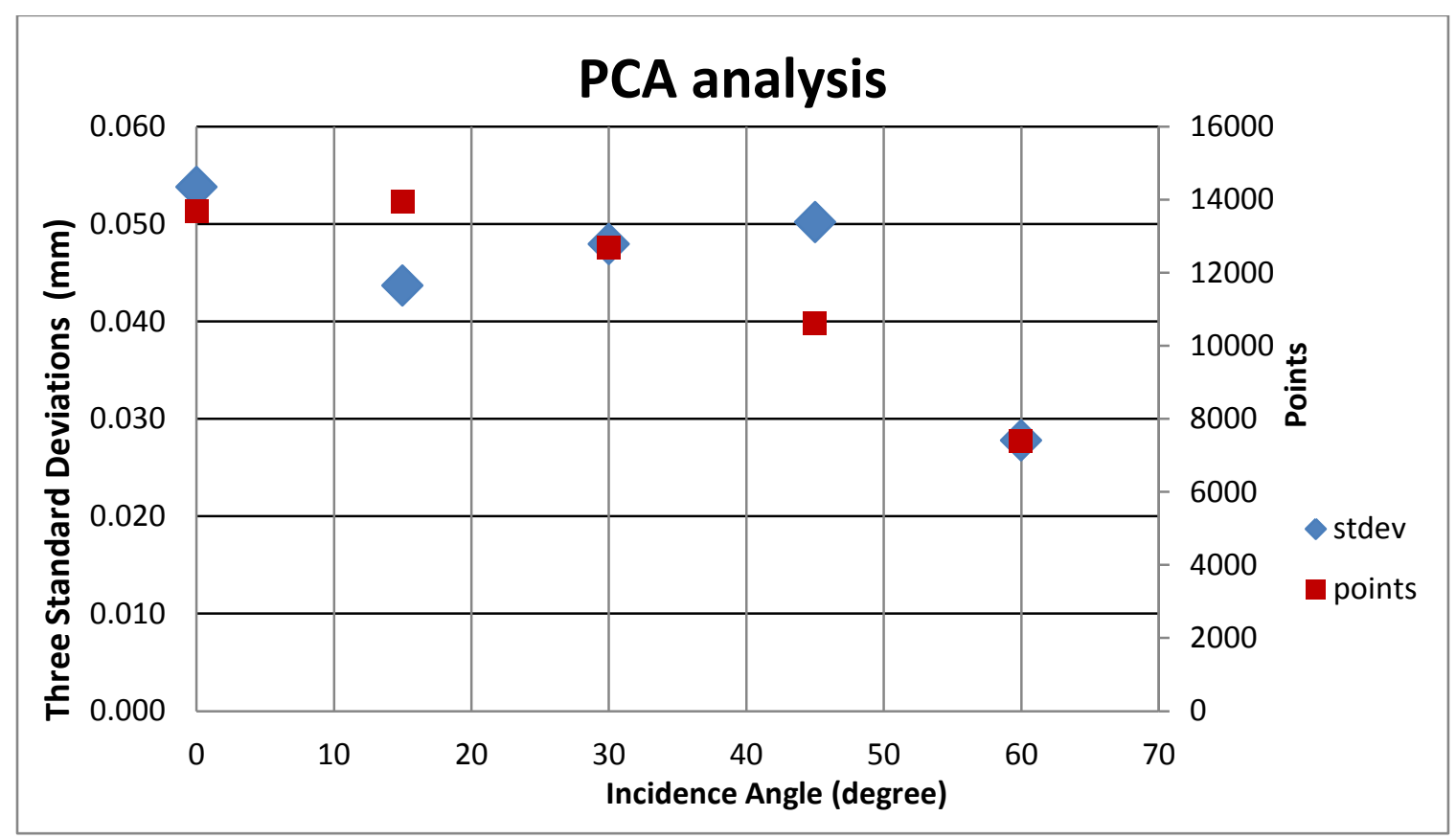

Figure 2.9: PCA using a constant radius of $15 \mathrm{~mm}$ for all images. The error, defined as three times the standard deviation, is on the left-hand vertical axis. The number of points used in the analysis is on the right-hand vertical axis.

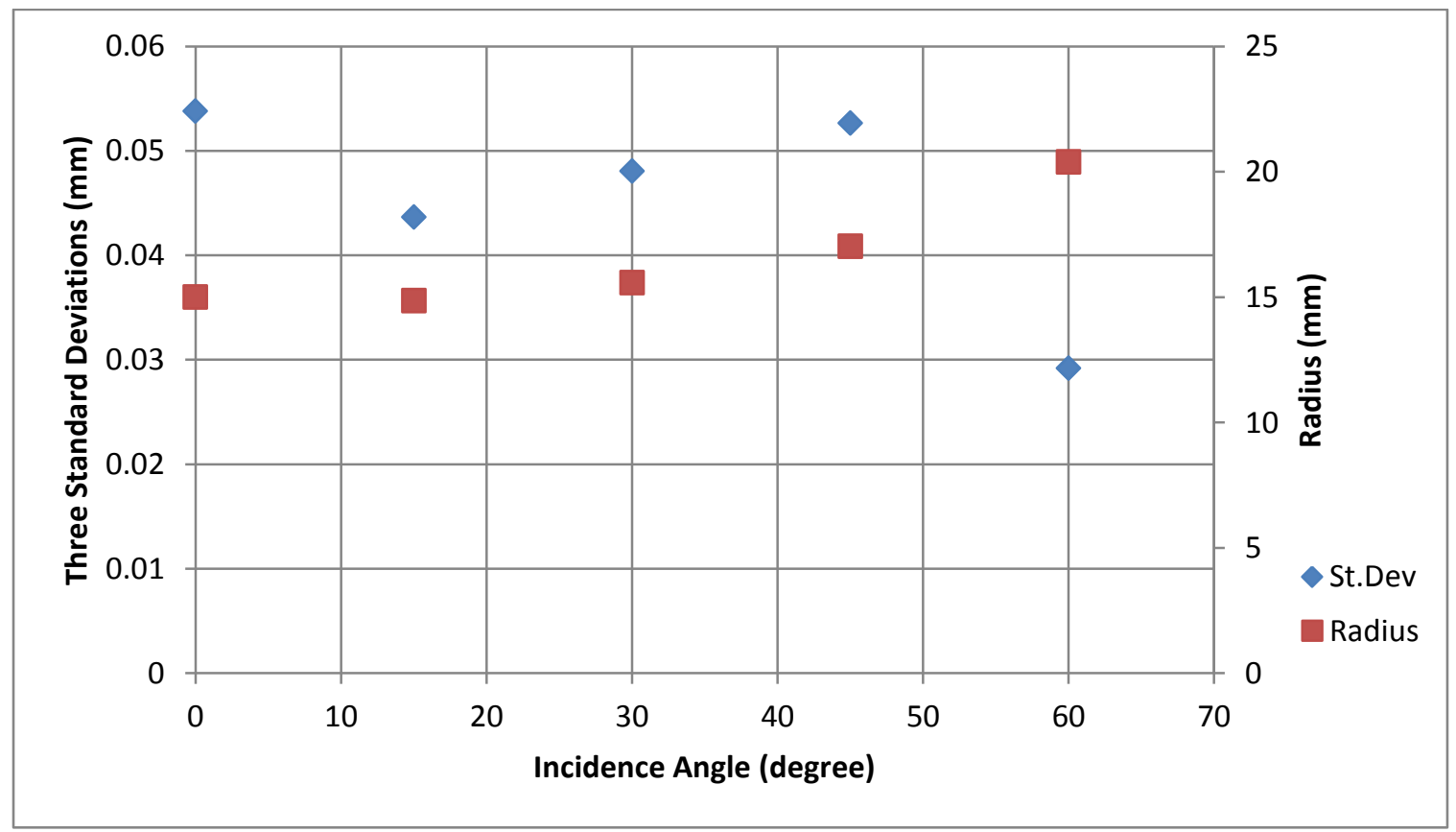

Figure 2.10: PCA with variable radius to include a consistent number of points. Standard deviation is on the left-hand vertical axis. The radius $(\mathrm{mm})$ used in the analysis is on the right-hand vertical axis highlights the region of interest on the metal plate that is selected for PCA analysis. 
depends on packing efficiency and humidity, however, so should be re-determined each time this method is performed (Macke et al., 2010). Volume can be estimated by comparing the mass of the sample in the air with the mass of the sample in the beads, and a known measurement volume (Equation 2.1).

$$
\text { Volume }_{\text {rock }}=\text { Volume }_{\text {vessel }} \times \frac{\text { Mass }_{\text {vessel,beads }}-\text { Mass }_{\text {vessel, beads,rock }}+\text { Mass }_{\text {rock }}}{\text { Mass }_{\text {vessel,beads }}-\text { Mass }_{\text {vessel }}}
$$

The formally calculated errors increase as the fragments get smaller. This method is only reliable for samples that are greater than $5 \mathrm{~cm}^{3}$ in volume (McCausland et al., 2011).

\subsection{2: Bead Measurement Process}

Prior to beginning any measurements, the beads were cycled back and forth between two containers. Termed 'warming up', this helped to insure that all the beads behaved the same, and shake loose any clumps that might have formed. The density of the beads can be found by filling a vessel of known volume, and measuring its mass. Next, calibration measurements were performed on a metal cylinder of known density. If the density found using the bead method is comparable to the known density, then the beads are assumed to be behaving in a fluid-like manner.

The procedure itself was fairly simple. The meteorite was placed into the vessel, a small glass cup, which was then filled to overflowing (Figure 2.11). Beads were placed in the bottom of the vessel so that the meteorite sample was completely 'submerged' in them, leaving as few empty spaces as possible. The vessel was quickly, yet gently, spun a half turn to encourage the beads to settle and minimize the space between them. The pouring of the beads and subsequent shaking needs to be performed in a repeatable way to maintain consistent results. 
Other settling methods used by Consolmagno et al. (2008) and Macke et al. (2010) make use of a vibrating platform to shake the vessel, or involve simply tapping its sides. A metal ruler was used to remove the excess beads from the top of the vessel. The vessel was then weighed on a scale (in this case, a triple beam balance) with an error of $\pm 0.05 \mathrm{~g}$. The procedure was repeated 10 times per sample, and an average taken. This method allows for rapid volume measurements, taking approximately 15-20 minutes per sample.

The greatest error in this measurement rests in the consistency of the pouring and shaking process (Consolmagno and Britt, 1998). In the Archimedean bead method, errors are introduced due to the non-fluid behavior of the beads. For example, the packing density of the beads could be inconsistent, producing unreliable results. The errors are notable when the sample is $<5 \mathrm{~cm}^{3}$ or $>50 \mathrm{~g}$ (Macke et al., 2010; Consolmagno et al., 2008). This effect is more problematic for small, dense samples, such as iron meteorites (Consolmagno and Britt, 1998). It appears that other than relatively humidity, no environmental factors, such as temperature or pressure, affect the bead measurements (Macke et al., 2010). 


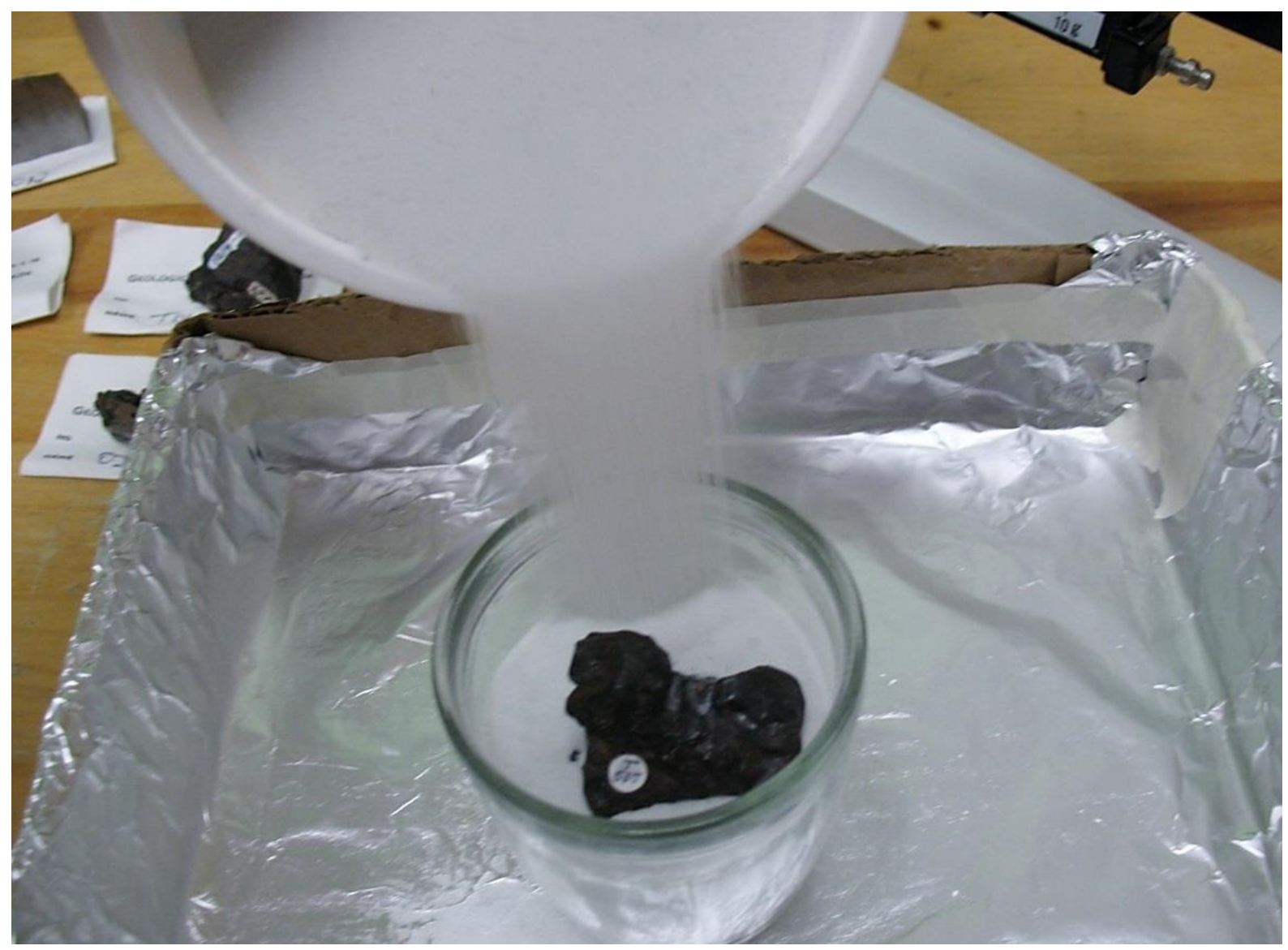

Figure 2.11: A 109.9g fragment of the Sikhote-Alin (\#T537) meteorite undergoing the Archimedean bead measurements. The diameter of the glass is $4.5 \mathrm{~cm}$. 


\section{Iron Meteorites}

\section{1: Samples}

A total of 41 iron meteorite samples, many of which are seen in Figure 3.1A (and Appendix A), were investigated in this study (Table 3.1). Samples were selected to represent a range of meteorite shapes and surface features, natural or not, to test the capabilities of the laser camera. The meteorites have rounded to angular edges, blocky to flat shapes, some of the samples possess cut and etched surfaces, and one sample has been cleaned of its fusion crust. The range of surface features imaged includes cracks, deep and shallow regmaglypts, and variable amounts of rust on the outer surfaces. Combinations of these features are present on most of the samples.

A range of sizes were imaged, from $8 \mathrm{~g}$ to $2250 \mathrm{~g}$. A variety of meteorite classes were represented by the samples studied. Of the 14 chemical classifications, the $A B$ or $A$ groups (IAB, $I I A B, I I A B$ and IVA) are the most abundant (Figure 3.2.A). Each has been represented by multiple fragments in this study (Figure 3.2.B). The other chemical groupings (e.g. IC, IIF, IIIE) and the ungrouped iron meteorites are unrepresented in this study.

\section{2: Archimedean Bead Measurements}

The density of relatively few iron meteorites had been measured with the Archimedean bead method, especially when compared to stony meteorites. Most of these density data can be found in Consolmagno and Britt (1998).

In this research project, only 28 out of the 41 samples studied were suitable for bead density measurements, due to size constraints. Archimedean bead measurements were carried out by 


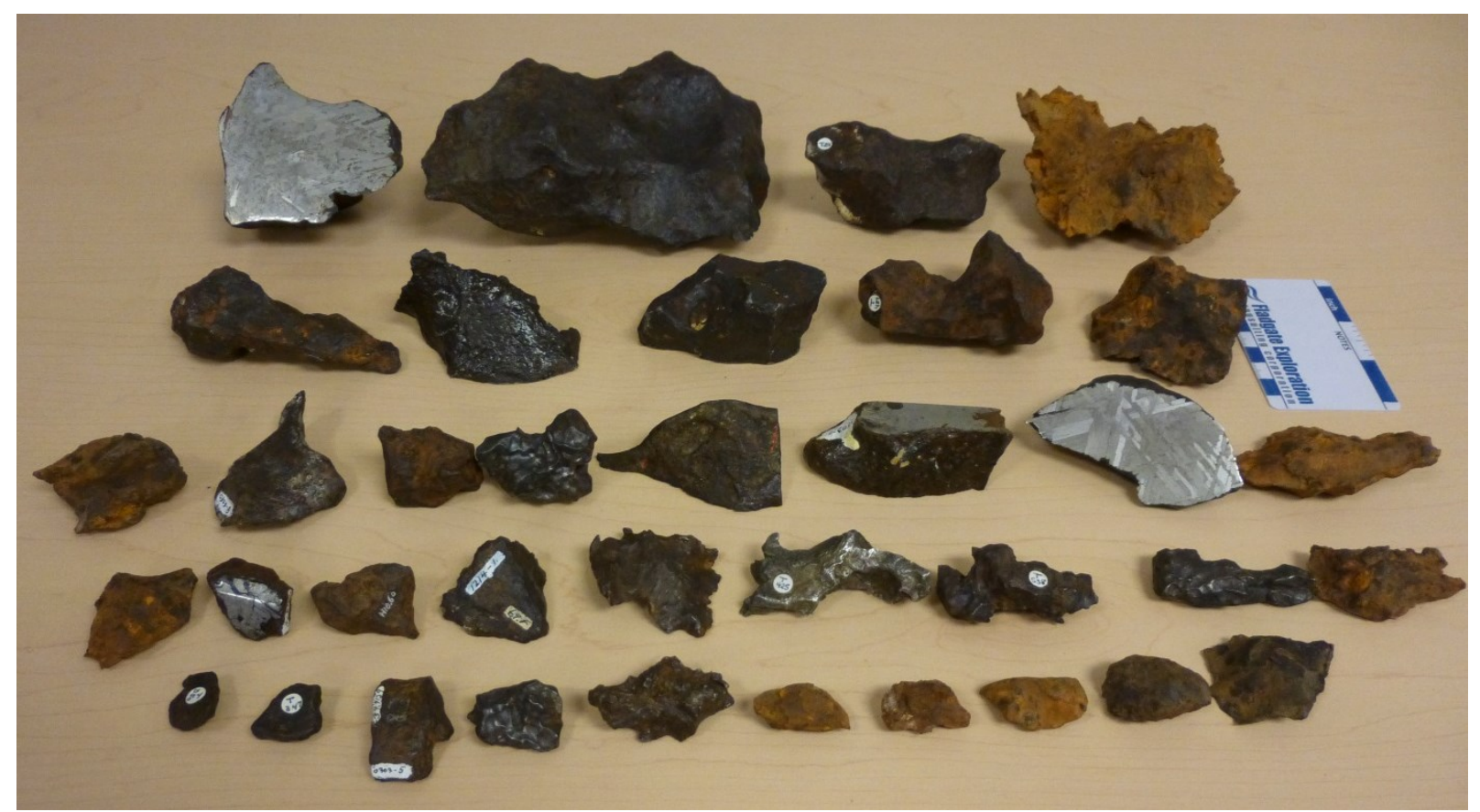

Figure 3.1: Samples provided by the National Meteorite collection of Canada, and the University of Alberta. This provides a good example of the range of material studied.

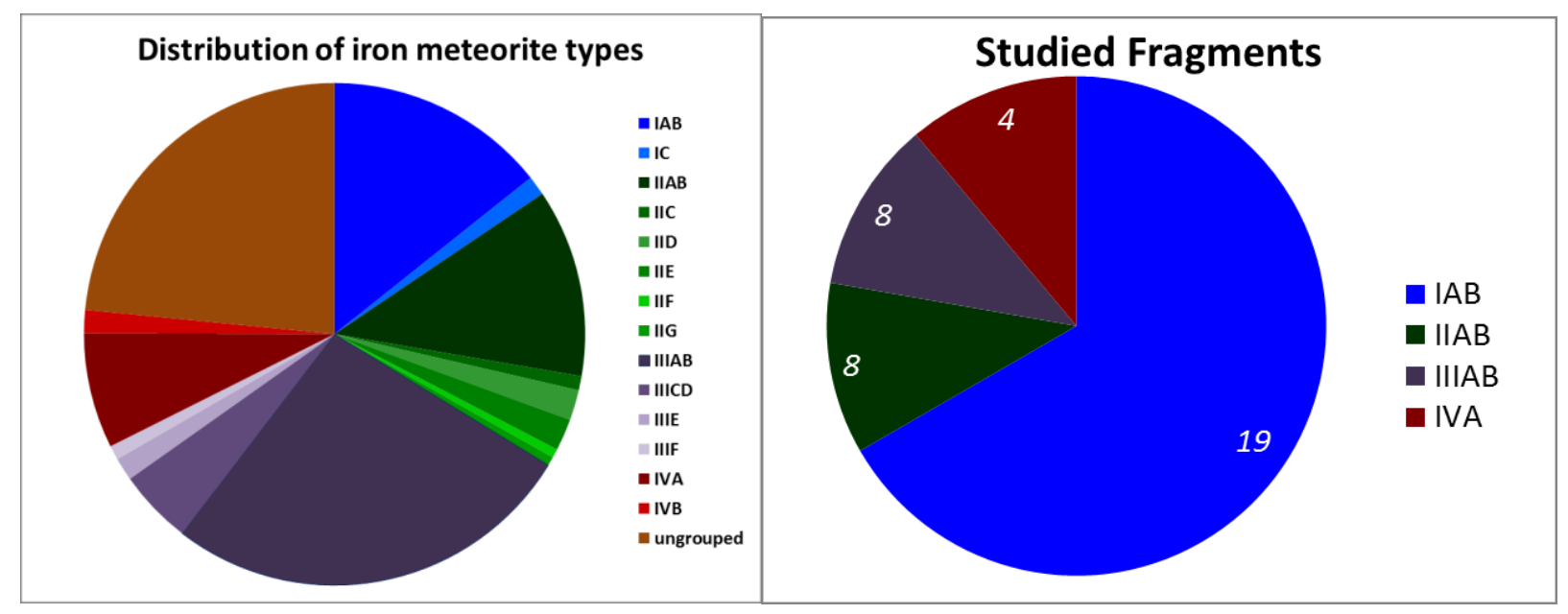

Figure 3.2: (Left) Distribution of iron meteorites in the world's collections and (right) samples investigated in this study (the number of samples for each class is indicated in italics). 
(UofA: University of Alberta; NMCC: National Meteorite Collection of Canada)

\begin{tabular}{|c|c|c|c|c|}
\hline Meteorite & Sample\# & $\begin{array}{l}\text { Mass } \\
(\mathrm{g})\end{array}$ & Class & Owner \\
\hline Campo de Cielo & phil1 & 243.80 & IA & Phil McCausland \\
\hline Campo Del Cielo & DBCC & 111.50 & IA & Dan Britt \\
\hline Canyon Diablo & $0303-2$ & 2250.00 & IAB-MG & NMCC \\
\hline Canyon Diablo & 34.5558 & 750.60 & IAB-MG & NMCC \\
\hline Canyon Diablo & T24 & 450.00 & IAB-MG & NMCC \\
\hline Canyon Diablo & h34.5604 & 444.75 & IAB-MG & NMCC \\
\hline Canyon Diablo & 0303-7 & 289.18 & IAB-MG & NMCC \\
\hline Canyon Diablo & UWO_005 & 213.30 & IAB-MG & Phil McCausland \\
\hline Canyon Diablo & 303.008 & 80.24 & IAB-MG & NMCC \\
\hline Canyon Diablo & 303.005 & 31.71 & IAB-MG & NMCC \\
\hline Gibeon & $0702-4$ & 194.49 & IVA & NMCC \\
\hline Gibeon & DBGB & 155.30 & IVA & Dan Britt \\
\hline Gibeon & 702.006 & 113.68 & IVA & NMCC \\
\hline Gibeon & UWO_012 & 11.25 & IVA & Phil McCausland \\
\hline Mundrabilla & PGB_016 & 56.93 & IAB-UNG & Phil McCausland \\
\hline Odessa & T489 & 396.79 & IAB-MG & NMCC \\
\hline Odessa & $1503-1$ & 206.55 & IAB-MG & NMCC \\
\hline Odessa & DBOD & 130.70 & IAB-MG & Dan Britt \\
\hline Odessa & OD897 & 86.63 & IAB-MG & NMCC \\
\hline Odessa & $\mathrm{H} 10.6$ & 44.19 & IAB-MG & NMCC \\
\hline Odessa & 1503.4 & 34.78 & IAB-MG & NMCC \\
\hline Sikote Alin & T537 & 109.94 & IIAB & NMCC \\
\hline Sikote Alin & T538 & 68.07 & IIAB & NMCC \\
\hline Sikote Alin & T425 & 67.62 & $\| A B$ & NMCC \\
\hline Sikote Alin & T483 & 54.46 & $\| A B$ & NMCC \\
\hline Sikote Alin & T539 & 50.49 & $\| A B$ & NMCC \\
\hline Sikote Alin & T487 & 42.76 & IIAB & NMCC \\
\hline Sikote Alin & T540 & 28.03 & IIAB & NMCC \\
\hline Sikhote-Alin & DBSA & 20.49 & $\| A B$ & Dan Britt \\
\hline Toluca & T17 & 264.80 & IAB-sLL & NMCC \\
\hline Toluca & 1214.001 & 59.01 & IAB-sLL & NMCC \\
\hline Whitecourt & $11617 / 10$ & 853.71 & IIIAB & University of Alberta \\
\hline Whitecourt & 148 & 253.22 & IIIAB & University of Alberta \\
\hline Whitecourt & $11617 / 185$ & 114.28 & IIIAB & University of Alberta \\
\hline Whitecourt & $11617 / 221$ & 44.49 & IIIAB & University of Alberta \\
\hline Whitecourt & 046 & 38.48 & IIIAB & University of Alberta \\
\hline Whitecourt & $11617 / 184$ & 22.57 & IIIAB & University of Alberta \\
\hline Whitecourt & 073 & 19.10 & IIIAB & University of Alberta \\
\hline Whitecourt & $11617 / 187$ & 18.31 & IIIAB & University of Alberta \\
\hline Zagora & T347 & 9.95 & IAB-ung & NMCC \\
\hline Zagora & T350 & 8.77 & IAB-ung & NMCC \\
\hline
\end{tabular}

Table 3.1: Data on iron meteorite samples 


\begin{tabular}{|c|c|c|c|c|c|c|c|c|}
\hline \multirow[b]{2}{*}{ Meteorite } & \multirow[b]{2}{*}{ Sample } & \multirow[b]{2}{*}{$\begin{array}{l}\text { Mass } \\
\text { (g) }\end{array}$} & \multicolumn{2}{|c|}{$\mathrm{CF}$} & \multicolumn{2}{|c|}{ MR } & \multicolumn{2}{|c|}{ Average } \\
\hline & & & $\begin{array}{l}\text { Density } \\
\left(\mathrm{g} / \mathrm{cm}^{3}\right)\end{array}$ & $\begin{array}{l}\text { Formal } \\
\text { Error } \\
\left(\mathrm{g} / \mathrm{cm}^{3}\right)\end{array}$ & $\begin{array}{l}\text { Density } \\
\left(\mathrm{g} / \mathrm{cm}^{3}\right)\end{array}$ & $\begin{array}{l}\text { Formal } \\
\text { Error } \\
\left(\mathrm{g} / \mathrm{cm}^{3}\right)\end{array}$ & $\begin{array}{l}\text { Density } \\
\left(\mathrm{g} / \mathrm{cm}^{3}\right)\end{array}$ & $\begin{array}{l}\text { Formal } \\
\text { Error } \\
\left(\mathrm{g} / \mathrm{cm}^{3}\right)\end{array}$ \\
\hline Campo de ceilo & phil1 & 243.80 & & & 7.26 & 0.22 & 7.26 & 0.22 \\
\hline Canyon Diablo & h34.5604 & 444.75 & & & 7.43 & 0.16 & 7.43 & 0.16 \\
\hline Canyon Diablo & UWO_005 & 213.30 & & & 7.44 & 0.25 & 7.44 & 0.25 \\
\hline Canyon Diablo & 303.008 & 80.24 & & & 7.74 & 0.39 & 7.74 & 0.39 \\
\hline Canyon Diablo & 303.005 & 31.71 & 7.08 & 0.43 & 7.00 & 0.62 & 7.04 & 0.519 \\
\hline Gibeon & $0702-4$ & 194.49 & & & 7.21 & 0.33 & 7.21 & 0.33 \\
\hline Gibeon & UWO_012 & 11.25 & 6.60 & 0.77 & 6.99 & 2.86 & 6.80 & 2.039 \\
\hline Mundrabilla & PGB_016 & 56.93 & 7.39 & 0.22 & 7.10 & 0.70 & 7.24 & 0.507 \\
\hline Odessa & 1503-1 & 206.55 & & & 7.11 & 0.14 & 7.11 & 0.14 \\
\hline Odessa & OD897 & 86.63 & 7.43 & 0.22 & 7.24 & 0.30 & 7.34 & 0.256 \\
\hline Odessa & $\mathrm{H} 10.5$ & 44.19 & 7.09 & 0.28 & 7.29 & 0.51 & 7.19 & 0.403 \\
\hline Odessa & 1503.4 & 34.78 & 7.09 & 0.39 & 7.26 & 0.59 & 7.17 & 0.488 \\
\hline Sikote Alin & T538 & 68.07 & 7.58 & 0.42 & 7.70 & 0.44 & 7.64 & 0.419 \\
\hline Sikote Alin & T425 & 67.62 & 7.47 & 0.32 & 8.35 & 0.66 & 7.91 & 0.506 \\
\hline Sikote Alin & T483 & 54.46 & 7.06 & 0.55 & 8.29 & 0.68 & 7.67 & 0.601 \\
\hline Sikote Alin & T539 & 50.49 & 7.71 & 0.60 & 7.82 & 0.62 & 7.77 & 0.592 \\
\hline Sikote Alin & T487 & 42.76 & & & 7.24 & 0.64 & 7.24 & 0.64 \\
\hline Sikote Alin & T540 & 28.03 & 7.24 & 0.88 & 7.96 & 1.15 & 7.60 & 0.997 \\
\hline Sikhote-Alin & DBSA & 20.49 & & & 7.63 & 1.09 & 7.63 & 1.09 \\
\hline Toluca & T17 & 264.80 & 7.47 & 0.16 & 7.07 & 0.14 & 7.27 & 0.145 \\
\hline Toluca & 1214.001 & 59.01 & 7.20 & 0.33 & 7.09 & 0.40 & 7.14 & 0.356 \\
\hline Whitecourt & $11617 / 221$ & 44.49 & 7.37 & 0.32 & 7.11 & 0.34 & 7.24 & 0.321 \\
\hline Whitecourt & 046 & 38.48 & 7.54 & 0.35 & 7.10 & 0.34 & 7.32 & 0.334 \\
\hline Whitecourt & $11617 / 184$ & 22.57 & 7.10 & 0.50 & 6.79 & 0.51 & 6.95 & 0.492 \\
\hline Whitecourt & 073 & 19.10 & 7.26 & 0.67 & 7.66 & 0.98 & 7.46 & 0.817 \\
\hline Whitecourt & $11617 / 187$ & 18.31 & 7.42 & 0.74 & 7.57 & 0.76 & 7.50 & 0.728 \\
\hline Zagora & T347 & 9.95 & 6.27 & 1.10 & 6.81 & 1.61 & 6.54 & 1.342 \\
\hline Zagora & T350 & 8.77 & 5.56 & 0.92 & 6.46 & 1.62 & 6.01 & 1.282 \\
\hline
\end{tabular}

Table 3.2: Archimedean bead measurements performed by two operators (CF and MR). Each operator performed at least 10 repeated measurements; the average and formal errors are reported. For samples studied by both operators, the data is averaged and the associated standard deviation is reported (two rightmost columns). 
two operators, following the method described in Section 2.2.2. At least ten repetitions of these measurements were carried out for each sample with statistics presented in Table 3.2. The Archimedean bead method calculates the volume of each sample using equation 2.1, designed by Consolmagno and Britt (1998). It relies on the operator knowing the volume of the vessel and assumes that the beads compact consistently with each repetition. Days with dry weather were chosen to reduce the potential stickiness of the beads due to humidity.

Table 3.2 records the formal error associated with the density data for samples studied by both operators. The formal error takes into account the errors associated with all the information used in calculating the volume, equation 3.1, and its propagation to the density calculation. This is largely because the volume of the sample relies heavily on how well the volume of the vessel is known. In doing so, the formal calculation includes the ratio in size between the sample measured and the reference volume because, as demonstrated by Consolmagno and Britt (1998), this contrast can affect the measurements. McCausland et al. (2011) and Macke et al. (2010) note that the formal error will also be affected by overall size of the sample, with those under $5 \mathrm{~cm}^{3}$ or over $50 \mathrm{~g}$ providing unreliable results.

The formal error of the density measurements for each operator is higher (averaged at $\pm 0.78 \mathrm{~g} / \mathrm{cm}^{3}$ for operator $\mathrm{CF}$ and $1.35 \mathrm{~g} / \mathrm{cm}^{3}$ for operator $\mathrm{MR}$ ) for the smaller samples, as expected (Figure 3.3). 


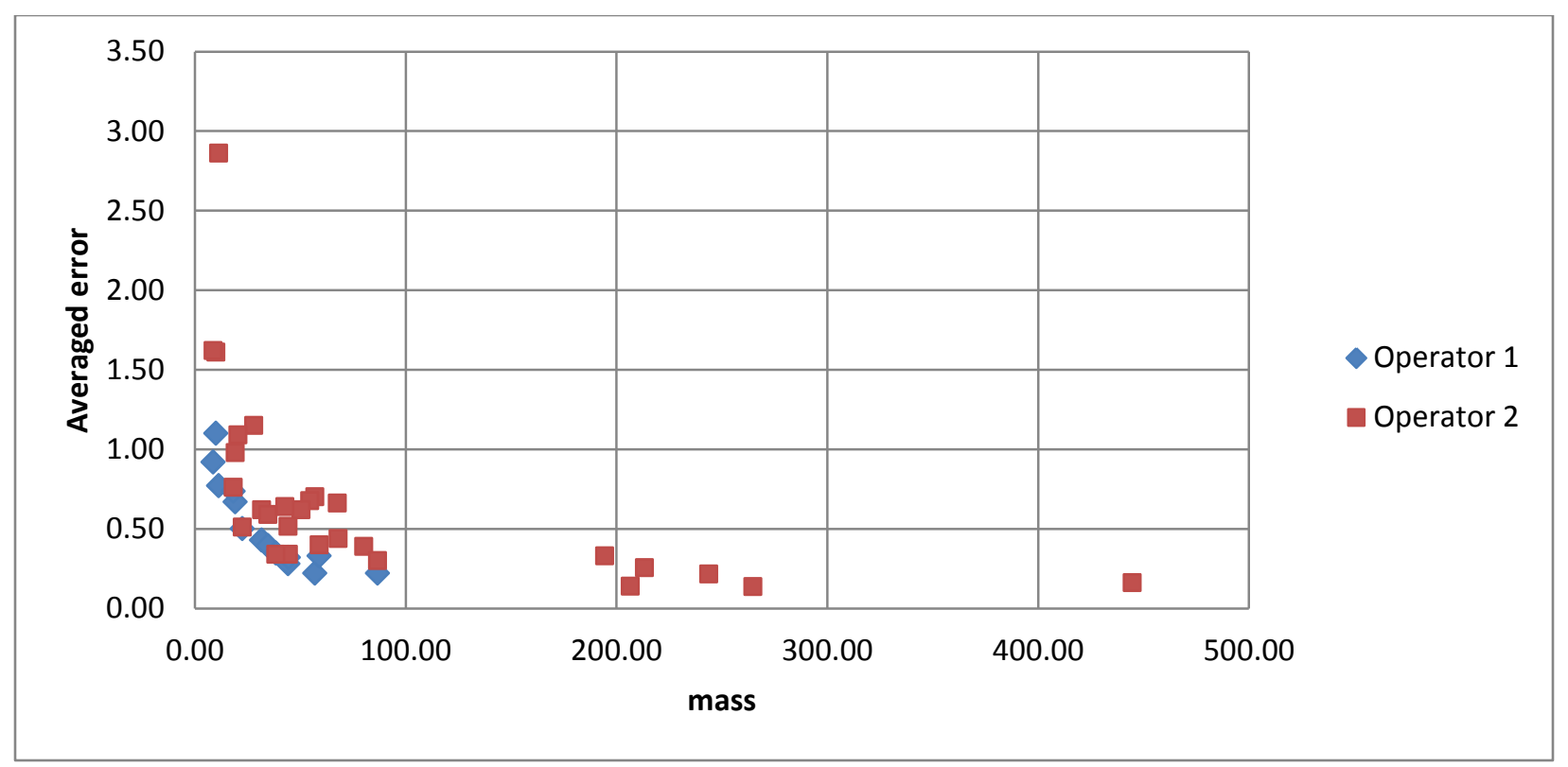

Figure 3.3: Average error associated with Archimedean bead density measurements versus sample mass for two operators (CF and MR).

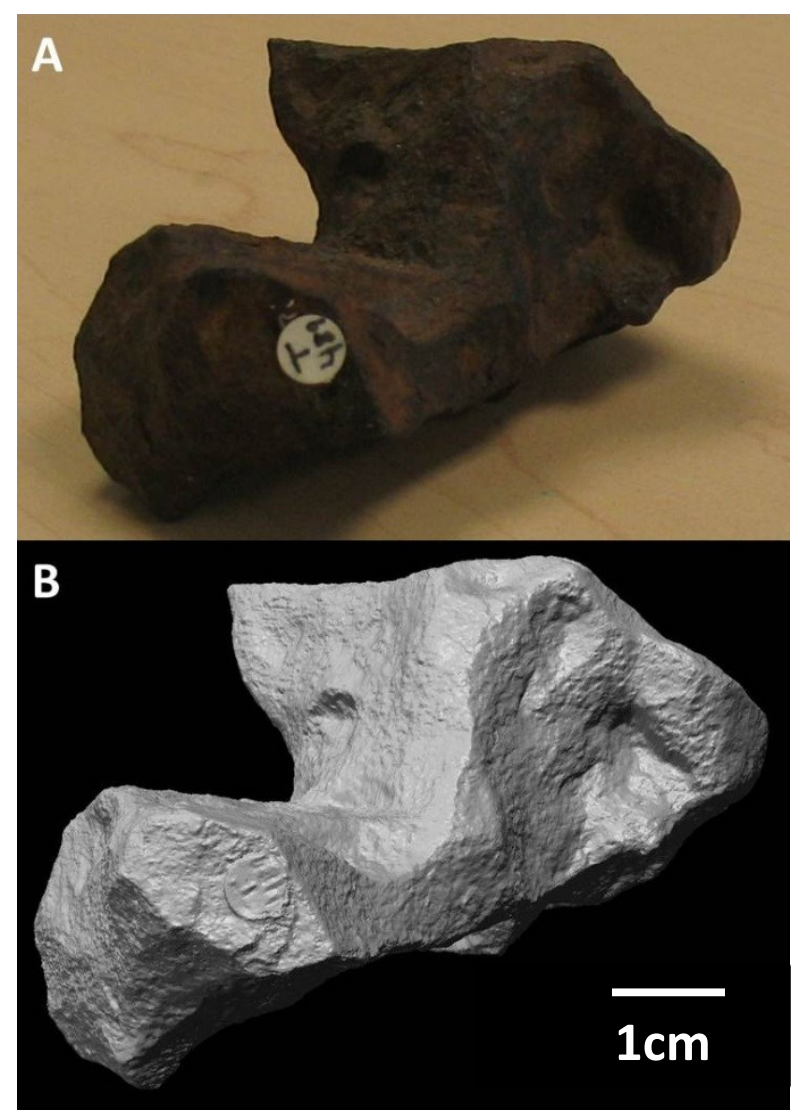

Figure 3.4: 396.79g Odessa (\#T489) fragment. A digital photograph is compared to the $3 \mathrm{D}$ model. The sample has a number of pits, a small crevasse and a distinctive shape, reminiscent of a dumbbell. The identification sticker in the model is semi legible. 


\section{3: Laser Imaging}

\subsection{1: Introduction}

There are a few benefits to the use of a laser camera when determining the density of iron meteorites. As for the Archimedean bead method, it is non-destructive. The full process of image acquisition and model creation, however, does take much longer per sample than the Archimedean process (McCausland et al., 2011). The laser imaging method for density measurements has progressed significantly since its inception in 2006 (Smith et al., 2006). The use of the Konica-Minolta VIVID 9i instead of the Laser Metrology System developed by Neptec Design Group has decreased the scanning time per image, allowing for 3 sets of 18 images to be acquired in less than 45 minutes. In comparison with the Archimedean bead method, laser imaging is applicable to a wider range of sample sizes, both smaller (as small as $0.5 \mathrm{~cm}^{3}$ ) and larger (sample size is not constrained by the size of the bead containers). It has been shown to produce more accurate results overall (McCausland et al., 2011).

\subsection{2: Image Acquisition and Model Assembly}

Once imported into Polyworks, it was possible to examine each image individually. Overall, the meteorites were imaged fairly easily, and there were few issues. The Konica-Minolta VIVID 9i proved to be very versatile; it was able to image a number of different natural and artificial surface features (some of which can be seen in Figures 3.4 and 3.5) on all 41 samples.

\section{Optical issues}

Many images featured the artifacts previously discussed in section 2.1 and were simply edited manually. For instance, present on many images was a slight distortion in the form of an 


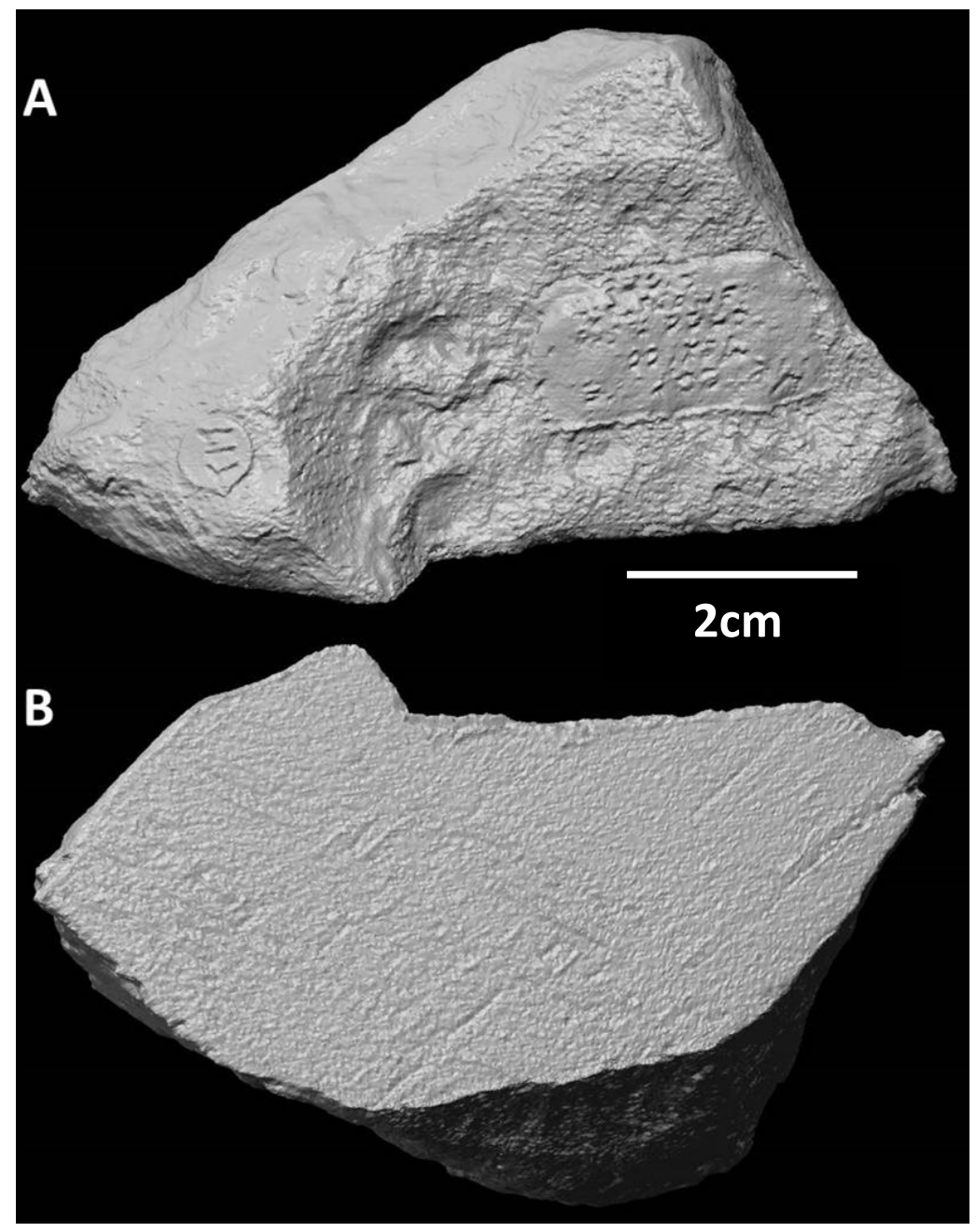

Figure 3.5

A. 3D image of the $264.8 \mathrm{~g}$ Toluca (\#T17) fragment. Natural and man made features can be seen in detail. Man made features are a legible sticker, "T17", on the left and a painted label on the right.

B. A cut surface of the fragment displays traces of mineral intergrowth.

C. Digital photograph of the Toluca fragment, showing the distinctive Widmanstätten pattern.

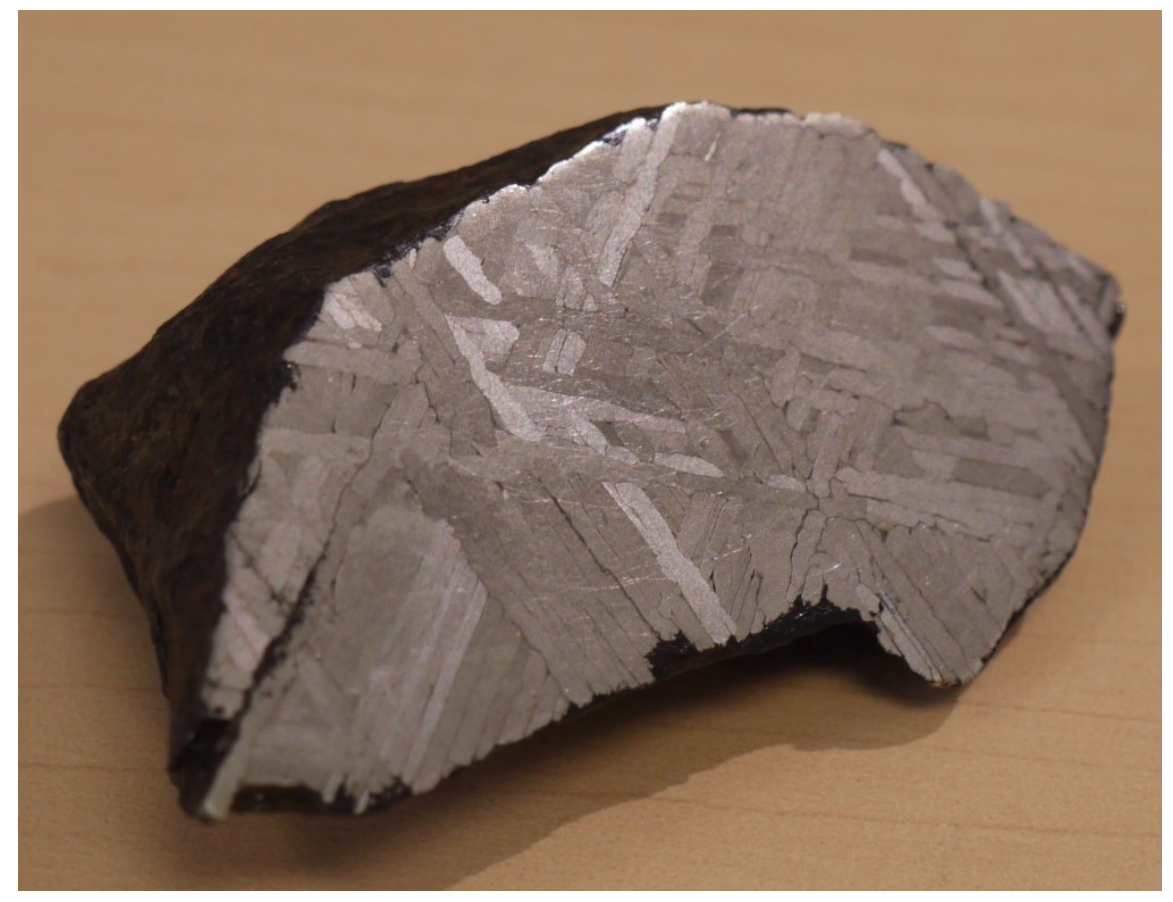


upturned edge due to field of view curvature. This artifact was most noticeable when the fragments surface was rounded. The stretched texture was also common when surfaces were imaged at an oblique angle. On the flat or cut surface of a meteorite, this effect became apparent for angles of incidence greater than $60^{\circ}$. The larger the angle, the more pronounced it was. Point scatter, the product of image noise, results in points that are not correctly placed within the ASCII cloud. Qualitatively, there was no relationship between the meteorite surface and their occurrence. Point scatter can be seen on curved surfaces or metallic cut faces, in the form of spurious features. Likewise, these scattered points did not occur at a set distance from the imaged surface. Typically, they occurred further away from the surface when a cut face was imaged. They occurred closer to the sample surface ( 1- $4 \mathrm{~mm})$ otherwise. A hole is produced when Polyworks uses the point cloud to produce a mesh of the cut face, while a protrusive artifact is observed for exterior surfaces.

Rust was present on a number of samples, especially those from the Whitecourt meteorite; however, rust is usually indistinguishable on the model surface as this layer is very thin and the surficial topography had not been altered by its presence. Also seen were holes in some images where the dark ablation surface on the sample had worn away revealing metallic iron-nickel underneath. Selecting a slightly different scene from the image library usually negated these issues (Fry et al., 2012).

The laser camera readily captured obvious surface features such as regmaglypts or cracks. The Odessa and Toluca samples (Figure 3.4B, Figure 3.5A) are seen to have rounded-angular edges, with visible depressions on the surface. Concave features on the surface of meteorites, such as pits, became progressively more difficult to image as their diameter decreased because 


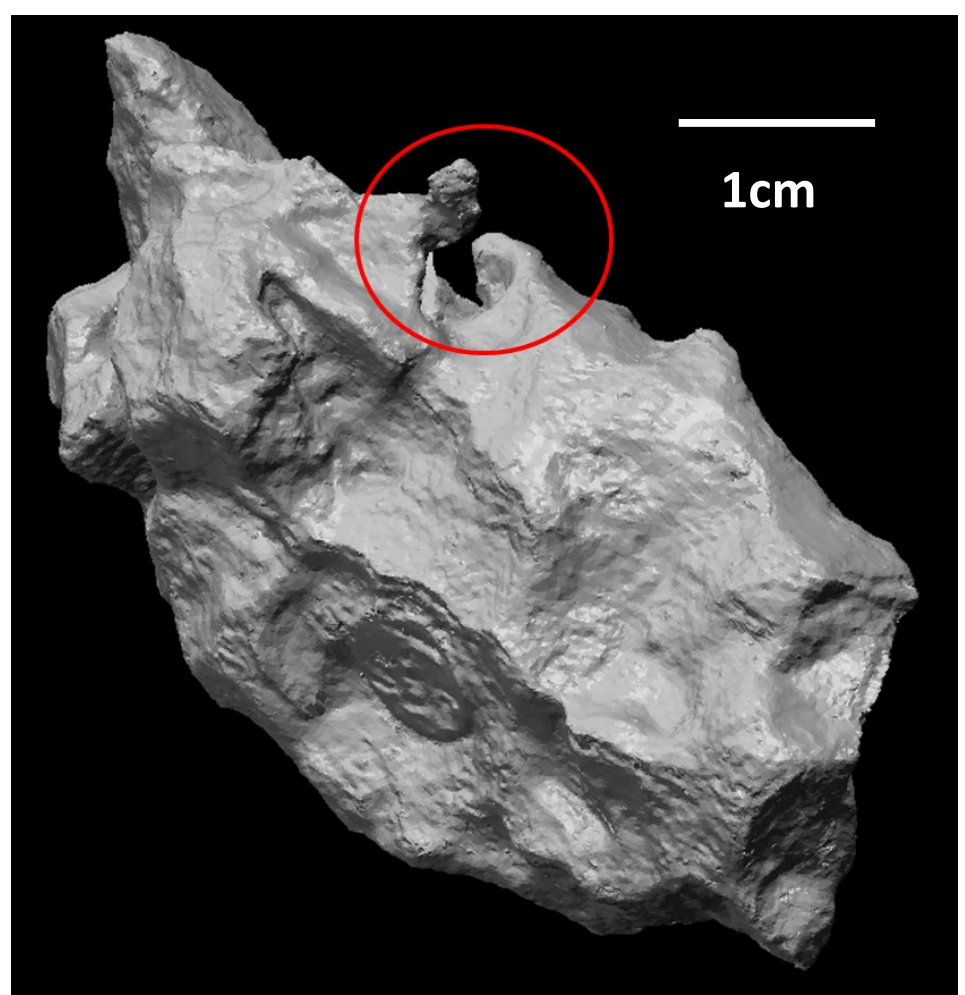

Figure 3.6: Shrapnel morphology of the 68.07g Sikhote-Alin (\#T538) meteorite fragment. A crevice formed by two twisted tendrils of iron proved challenging to image when compared to the rest of the surface, which is covered in regmaglypts.

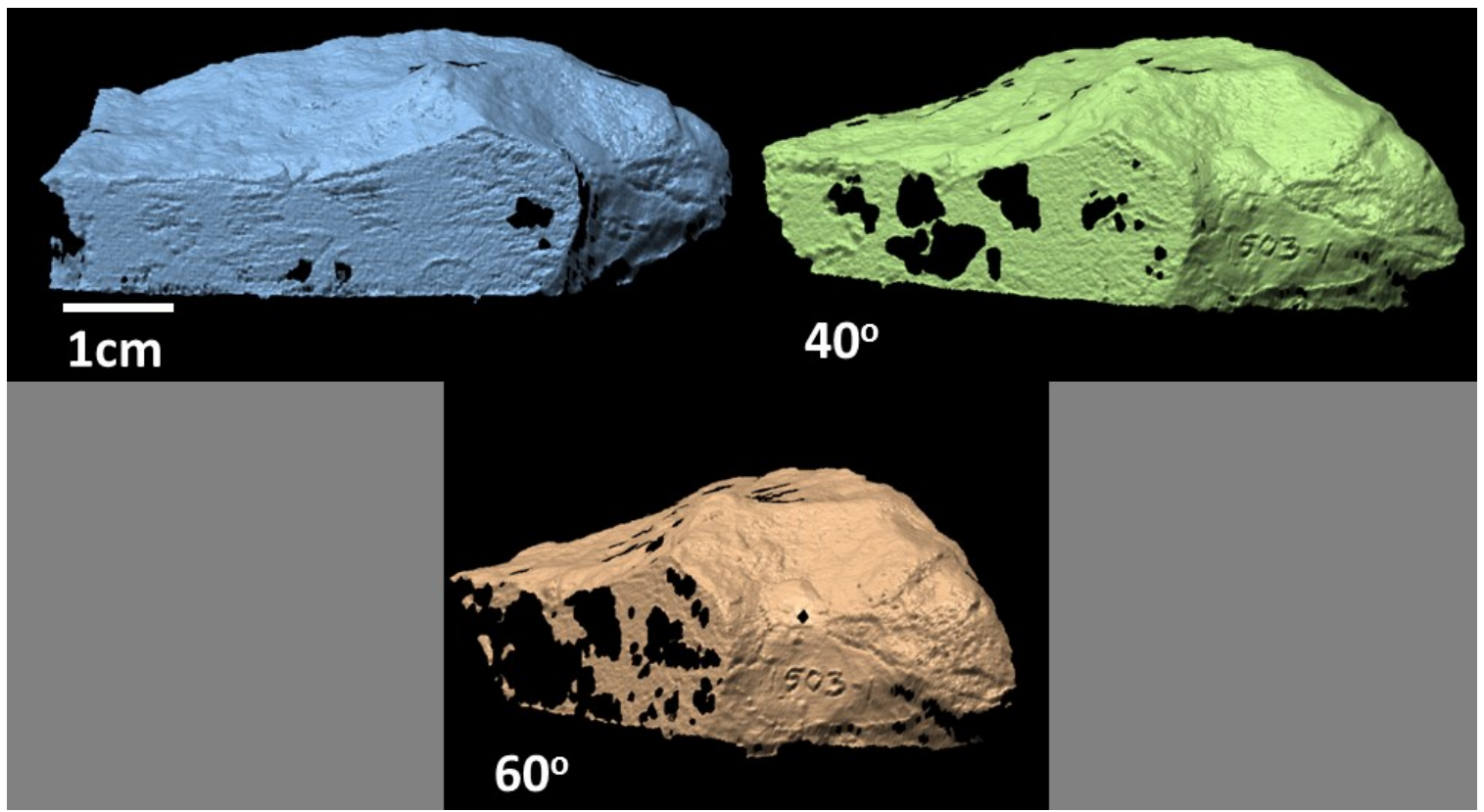

Figure 3.7: The 206.55g sample of Odessa (\#1503-1) with three cut surfaces. Gaps in the point cloud appear and become larger as the sample is rotated. 
of occlusion (the edges of the pit block the line of sight of the laser beam). A narrow deep pit is harder to image than a wide deep pit because of this effect. Likewise, a crack that appears solely as a surficial trace is less distinguishable than a crack that has split apart (crevice).

Some of the meteorite fragments have a twisted, shrapnel-like morphology. At times this shape resulted in a feature, such as a sharp jagged edge or a twisted tendril that needed careful attention during imaging and alignment. The tendril morphology can pose a challenge when the iron offshoot is close to the surface of the main body. A pit or crevice can form that is difficult for the laser to properly reach. An example of this is shown in Figure 3.6.

\section{Artificial surfaces and features}

Imaging some metallic surfaces was challenging. Cut faces were captured easily when perpendicular to the camera; when at an angle to the camera, they would often fail to return signal.

Special attention had to be paid to samples with sharp edges (most often artificial; less often natural) or cut faces. If an edge comes to a sharp point, there is a distinct possibility that the same small gap will occur in several images. This is exacerbated by the need to trim the edges of each image and to remove areas with a stretched texture, or that are upturned. In some cases, both sides of the edge exhibited gaps. It is possible to fill in the holes automatically; however, this approach might lead to an under-representation of the volume of the fragment, through the production of a blocky edge. One of the samples (the 206.55g fragment of Odessa, \#1503-1, Figure 3.7) possessed three adjacent cut faces, at right angles to each other. Only one cut face would generate points in the cloud when multiple cut faces where in view. The oblique 
angle between the laser and the flat surface was the potential cause of this. Gaps would begin to appear in the cut face when angled over $40^{\circ}$ (Figure 3.7) with respect to the camera. This only occurred for some of the cut faces of the different meteorites, usually ones that were vertical when imaged.

Artificial features present were stickers and painted on labels. Writing on the stickers (sample numbers) was legible, such as in Figure 3.4A, 3.5A. The painted labels, while visible, were less so. The visibility of these features is probably a byproduct of bleeding.

A $264.8 \mathrm{~g}$ sample of Toluca (\#T17) possessed a cut face that had been etched with acid, and displayed a classic example of the Widmanstätten pattern(Figure $3.5 \mathrm{C}$ ). This pattern, caused by kamacite and taenite intergrowth, has no physical relief on the cut surface when felt by hand. This pattern turned up in the final model as a faint network of crisscrossing lines (Figure $3.5 \mathrm{~B}$ ). How did the laser camera capture these very subtle features, which hardly have any physical relief? It might be that these features have a very small amount of relief associated to them, close to the noise threshold of $0.054 \mathrm{~mm}$, which allows for partial detection. Or, as for the text on the stickers, it might also be the result of bleeding produced by a change in intensity across its surface.

A 67.62g Sikhote-Alin (\#T425) fragment posed a special challenge. Aside from a shrapnel shape, it also possessed a cleaned surface. Brushing the fusion crust from the surface exposed the metallic iron underneath and left tool marks behind. Many of the images that were produced had a high number of spurious protrusions, more so than any of the other meteorite samples. The protrusions occurred predominately on the edges of this sample, and images often possessed the stretched texture. While volumetrically accurate, the surficial texture of 


\begin{tabular}{|c|c|c|c|c|c|c|c|c|c|}
\hline \multirow[b]{2}{*}{ Meteorite } & \multirow[b]{2}{*}{ Sample\# } & \multirow[b]{2}{*}{$\begin{array}{l}\text { Mass } \\
\text { (g) }\end{array}$} & \multirow[b]{2}{*}{$\begin{array}{l}\text { Image } \\
\text { Library }\end{array}$} & \multicolumn{5}{|c|}{ 3D Laser Imaging bulk density } & \multirow{2}{*}{$\begin{array}{c}\text { Coeff. } \\
\text { of } \\
\text { Vari. } \\
(\%)\end{array}$} \\
\hline & & & & CF & MR & TKM & Average & $\begin{array}{l}\text { Standard } \\
\text { Deviation }\end{array}$ & \\
\hline Campo de Cielo & phil1 & 243.80 & 57 & 7.40 & & 7.45 & 7.43 & 0.03 & 0.47 \\
\hline Campo Del Cielo & $\mathrm{DBCC}$ & 111.50 & 57 & 7.59 & 7.53 & & 7.56 & 0.05 & 0.60 \\
\hline Canyon Diablo & 0303-2 & 2250.00 & 41 & 7.73 & & & 7.73 & & \\
\hline Canyon Diablo & 34.5558 & 750.60 & 54 & 7.50 & & & 7.50 & & \\
\hline Canyon Diablo & T24 & 450.00 & 54 & 7.28 & & & 7.28 & & \\
\hline Canyon Diablo & h34.5604 & 444.75 & 57 & 7.60 & 7.61 & 7.62 & 7.61 & 0.01 & 0.13 \\
\hline Canyon Diablo & 0303-7 & 289.18 & 57 & 7.31 & 7.37 & 7.30 & 7.33 & 0.04 & 0.49 \\
\hline Canyon Diablo & UWO_005 & 213.30 & 32 & 7.61 & 7.61 & 7.69 & 7.64 & 0.04 & 0.59 \\
\hline Canyon Diablo & 303.008 & 80.24 & 30 & 7.14 & & 7.38 & 7.26 & 0.17 & 2.33 \\
\hline Canyon Diablo & 303.005 & 31.71 & 57 & 7.34 & 7.26 & 7.26 & 7.29 & 0.05 & 0.62 \\
\hline Gibeon & 0702-4 & 194.49 & 63 & 7.25 & 7.38 & 7.42 & 7.35 & 0.09 & 1.21 \\
\hline Gibeon & DBGB & 155.30 & 39 & 7.72 & 7.63 & 7.68 & 7.68 & 0.04 & 0.58 \\
\hline Gibeon & 702.006 & 113.68 & 59 & 7.56 & 7.88 & 7.71 & 7.72 & 0.16 & 2.09 \\
\hline Gibeon & UWO_012 & 11.25 & 42 & 7.22 & 7.01 & 6.93 & 7.05 & 0.15 & 2.09 \\
\hline Mundrabilla & PGB_016 & 56.93 & 32 & 7.31 & 7.30 & 7.34 & 7.32 & 0.02 & 0.27 \\
\hline Odessa & T489 & 396.79 & 44 & 7.42 & 7.36 & 7.40 & 7.39 & 0.03 & 0.42 \\
\hline Odessa & $1503-1$ & 206.55 & 61 & 7.19 & 7.24 & 7.17 & 7.20 & 0.04 & 0.49 \\
\hline Odessa & DBOD & 130.70 & 36 & 7.54 & 7.40 & 7.45 & 7.46 & 0.07 & 0.92 \\
\hline Odessa & OD897 & 86.63 & 54 & 7.24 & 7.27 & 7.34 & 7.28 & 0.05 & 0.74 \\
\hline Odessa & H10.6 & 44.19 & 54 & 7.04 & 7.02 & 7.23 & 7.10 & 0.12 & 1.63 \\
\hline Odessa & 1503.4 & 34.78 & 59 & 6.99 & 6.91 & 7.03 & 6.98 & 0.07 & 0.93 \\
\hline Sikhote Alin & T537 & 109.94 & 62 & 7.65 & 7.74 & 7.66 & 7.69 & 0.05 & 0.60 \\
\hline Sikhote Alin & T538 & 68.07 & 55 & 7.53 & 7.44 & 7.60 & 7.52 & 0.08 & 1.04 \\
\hline Sikhote Alin & T425 & 67.62 & 54 & 7.99 & 8.03 & 7.78 & 7.93 & 0.13 & 1.69 \\
\hline Sikhote Alin & T483 & 54.46 & 53 & 7.69 & 7.67 & 7.73 & 7.70 & 0.03 & 0.39 \\
\hline Sikhote Alin & T539 & 50.49 & 44 & 7.76 & 7.78 & 7.83 & 7.79 & 0.03 & 0.44 \\
\hline Sikhote Alin & T487 & 42.76 & 58 & 7.50 & 7.50 & 7.56 & 7.52 & 0.04 & 0.48 \\
\hline Sikhote Alin & T540 & 28.03 & 59 & 7.77 & 7.77 & 7.79 & 7.77 & 0.01 & 0.13 \\
\hline Sikhote-Alin & DBSA & 20.49 & 54 & 7.69 & 7.25 & 7.70 & 7.55 & 0.26 & 3.43 \\
\hline Toluca & T17 & 264.80 & 60 & 7.46 & 7.35 & 7.43 & 7.41 & 0.05 & 0.73 \\
\hline Toluca & 1214.001 & 59.01 & 54 & 7.02 & 6.96 & 7.03 & 7.00 & 0.03 & 0.49 \\
\hline Whitecourt & $11617 / 10$ & 853.71 & 31 & 7.37 & & 7.39 & 7.38 & 0.01 & 0.18 \\
\hline Whitecourt & 148 & 253.22 & 56 & 7.56 & & 7.50 & 7.53 & 0.04 & 0.54 \\
\hline Whitecourt & $11617 / 185$ & 114.28 & 52 & 7.35 & & 7.45 & 7.40 & 0.07 & 0.94 \\
\hline Whitecourt & $11617 / 221$ & 44.49 & 55 & 7.38 & & 7.38 & 7.38 & 0.00 & 0.01 \\
\hline Whitecourt & 046 & 38.48 & 54 & 7.50 & & 7.53 & 7.52 & 0.03 & 0.35 \\
\hline Whitecourt & $11617 / 184$ & 22.57 & 54 & 7.44 & & 7.53 & 7.49 & 0.06 & 0.79 \\
\hline Whitecourt & 073 & 19.10 & 56 & 7.34 & & 7.41 & 7.38 & 0.05 & 0.70 \\
\hline Whitecourt & $11617 / 187$ & 18.31 & 54 & 7.57 & & 7.56 & 7.56 & 0.01 & 0.11 \\
\hline Zagora & T347 & 9.95 & 54 & 6.61 & 6.37 & 6.55 & 6.51 & 0.13 & 1.96 \\
\hline Zagora & T350 & 8.77 & 44 & 5.97 & 5.80 & 5.87 & 5.88 & 0.09 & 1.50 \\
\hline
\end{tabular}

Table 3.3: Laser-derived densities calculated using 3D models built by three separate operators. 
the final model was not true to the original sample. Although they were edited, the images still produced a number of small scale artifacts that distorted the model.

Less than a third (15 on average) of these images was used in the final model construction. The time needed to assemble and edit the model varied from 30 minutes to 2 hours based on the complexity of the sample.

\subsubsection{Error Analysis}

Inter-operator variability

Three experienced operators assembled 3D models of many of the samples in this study. Each operator had the ability to independently choose any image from the image library (Table 3.3). Overall, each operator used an average of 15 (CF), 11 (MR), and 13 (TM) images. There was a $42 \%$ percent chance of an images being used by at least 2 of the three operators. The coefficient of variation is calculated to assess the difference in the results produced by each operator. The coefficient of variation is defined as the ratio of the standard deviation of the densities determined by each operator to the mean. This value ranges from $0.11-3.43 \%$, with an average of $0.93 \%$. This shows that $3 \mathrm{D}$ model building is a very repeatable process.

Impact of sample size and shape on error

Figure 3.8 looks to establish a relationship between the coefficient of variation and sample size and shape. Figures 3.8 A-C investigate volume, surface area and mass, all of which relate to the physical size of the sample. Each graph shows that as the sample size decreases, the coefficient of variation increases non-linearly. Finally, Figure 3.8 D is the coefficient of variation 
vs surface area/spherical equivalent index. This index is meant to represent the complexity of the sample shape, by comparing the surface area of a sample to the surface area of a sphere with an equivalent volume. If the index is equal to one, then the meteorite would be a perfect sphere. Increasing the index represents an increase in the complexity of the shape, accounting for any features that may be seen on the meteorite, such as regmaglypts or shrapnel tendrils. For the samples imaged, the index is between 1.25 and 1.98. In Figure 3.8 D, there does not appear to be a correlation between the coefficient of variation and the index. The sample shapes with the highest index are those that have a disc shape (e.g. 54.46g Sikhote-Alin (\#T483), pictured in Appendix A, has the highest index at 1.98), while samples with a number of regmagyplts plot next (1.5-1.7)( e.g. 109.94g Sikhote-Alin, T537, pictured in Appendix A). In general, samples with a rounded shape have lower indices than the more angular samples. Most samples with cut faces were otherwise fairly rounded, plotting between 1.35-1.45 (e.g. the two Zagora fragments). Two cut samples plotted at 1.69 e.g. 194.49g Gibeon (\#07024)fragment, pictured in Appendix A), likely due to having some very sharp edges. Overall, the data gathered in this study shows that measurement error, as expressed by the coefficient of variation, is mostly associated to physical size rather than shape.

\section{4: Comparison between densities derived using the Archimedean Bead Method and Laser Imaging}

The Archimedean bead method and 3D laser imaging have provided consistent density measurements in past studies with an average difference of $0.46 \%$ (using the VIVID 9i digitizer) 

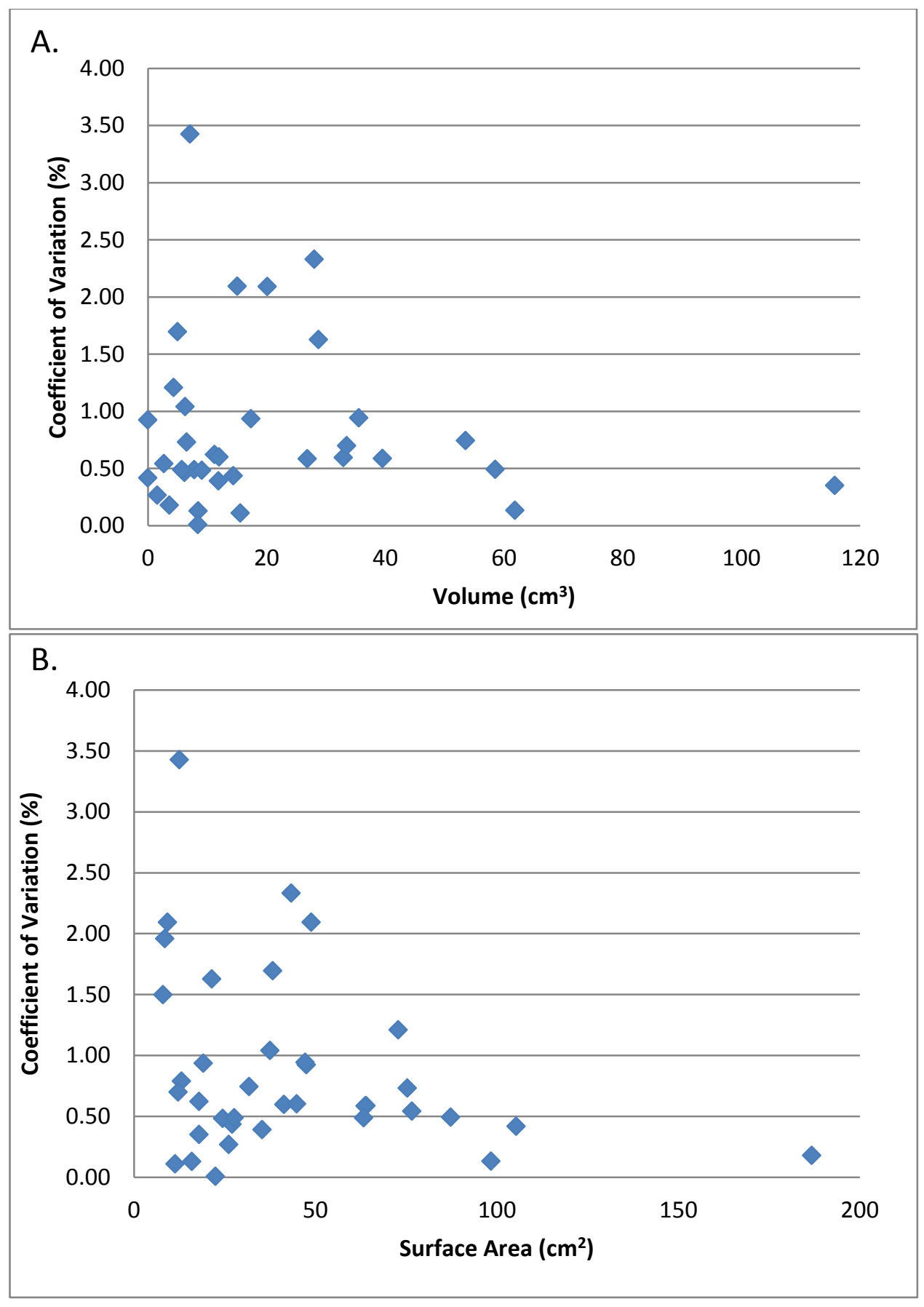

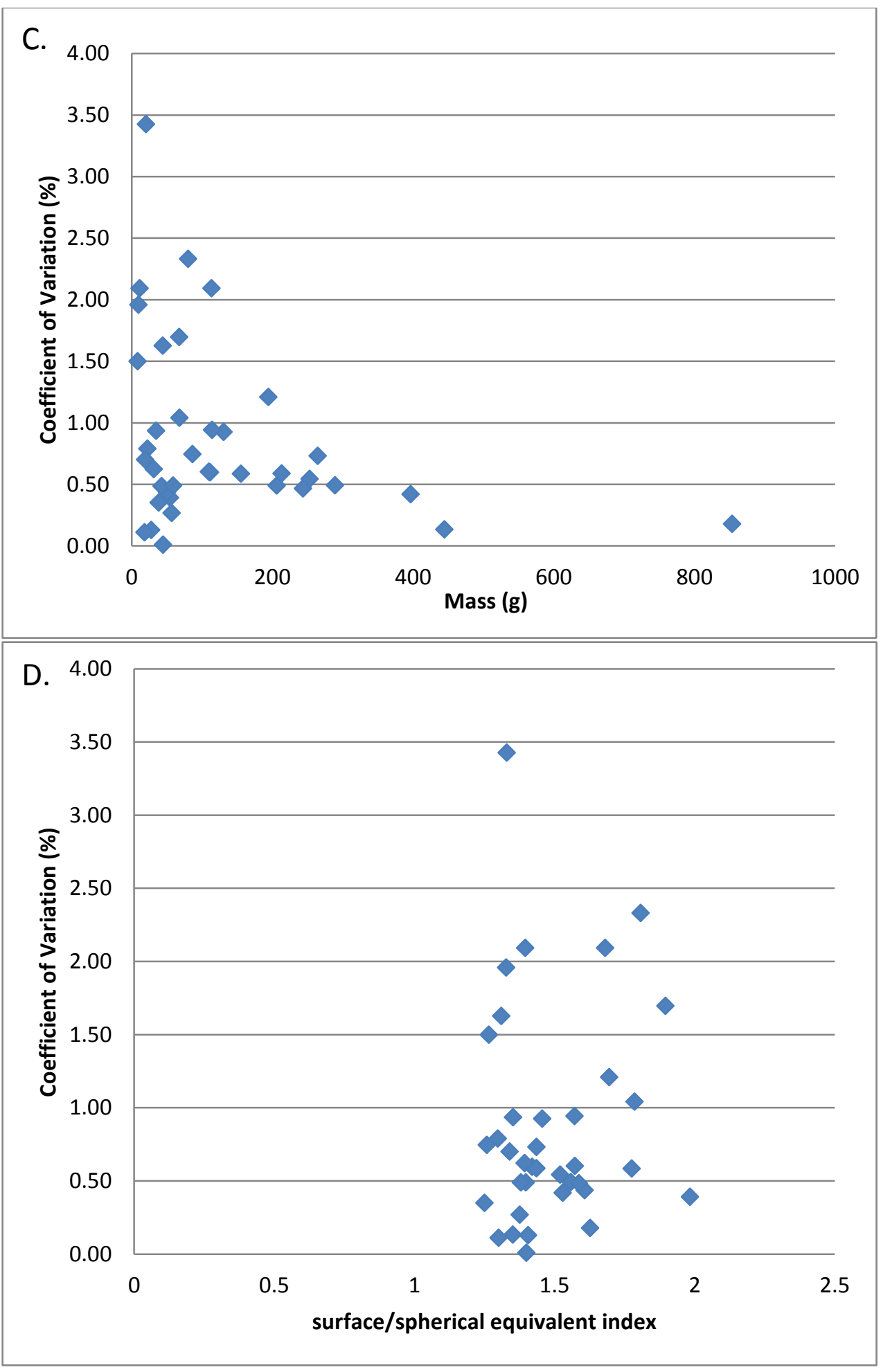

Figure 3.8: Coefficient of variation associated with laser-derived density measurements versus (A) volume (average of three operators); (B) surface area (CF models only); (C) mass; and (D) surface area/spherical equivalent index (CF models only). 


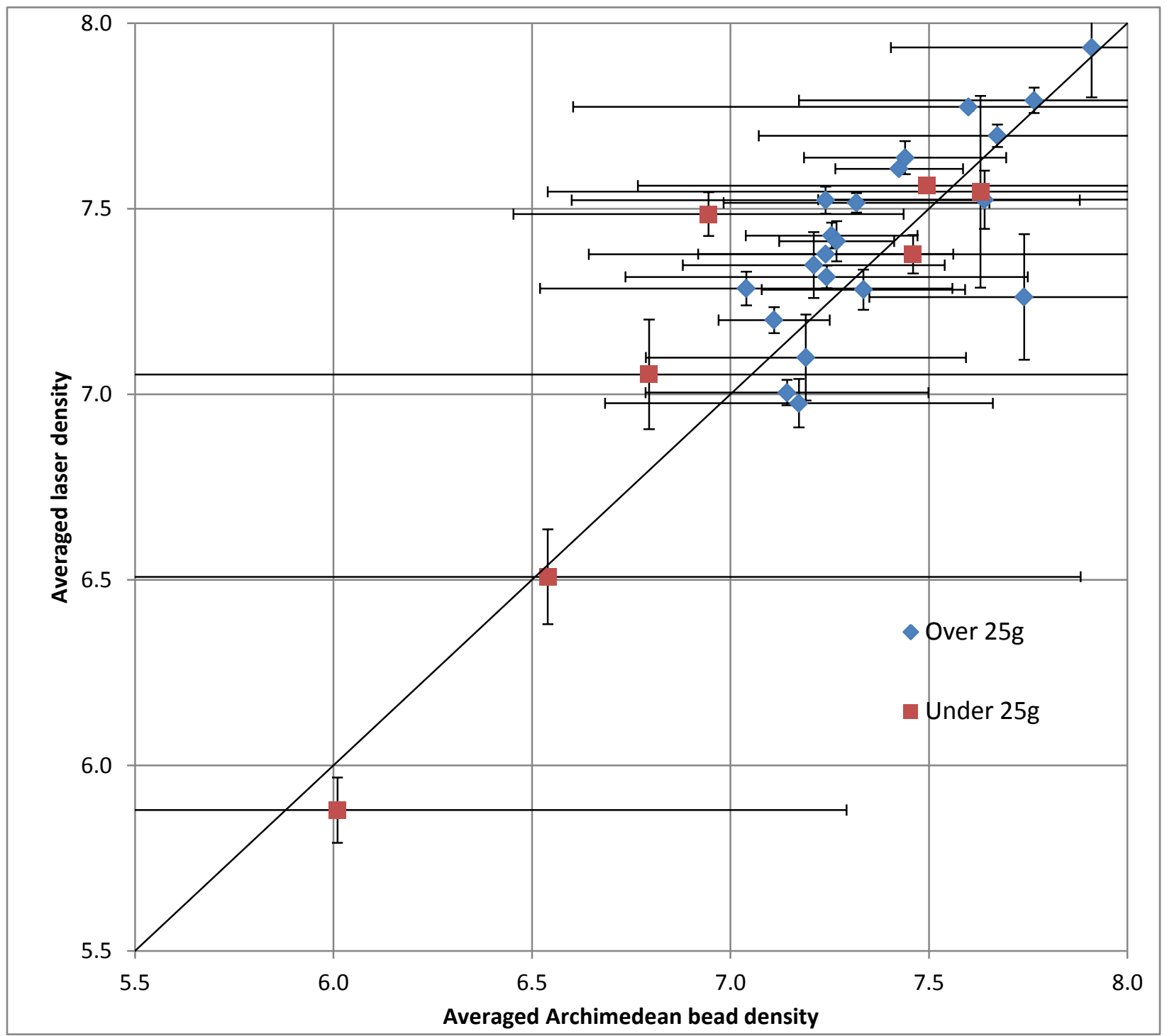

Figure 3.9: Laser-derived density versus Archimedean bead density for 28 iron meteorite fragments, plotted with respect to a 1:1 reference line. The Archimedean errors are larger for smaller samples. 
(Fry et al., 2013). Figure 3.9 is a direct comparison for the 28 fragments that were measured using both methods in this study. The data mainly clusters around the 1:1 line. The 3 samples with the highest variation were also among the smallest, all $<11 \mathrm{~g}$, with Archimedean bead density errors that all exceed $1.28 \mathrm{~g} / \mathrm{cm}^{3}$. These outliers will not be included in further analysis.

Following the method used in McCausland et al. (2011), equation 3.2

$$
\mathrm{R}=\frac{\rho_{\text {bead }}-\rho_{\text {laser }}}{\rho_{\text {bead }}}
$$

calculates the coefficient of correlation $\mathrm{R}$, expressed as a percentage. The coefficient of correlation is used to describe the difference in results between the two methods. A negative coefficient denotes that the laser density is larger than the bead density. These data are found in table 3.4. There is an average $2.13 \%$ (maximum $7.78 \%$ ) difference between the laser-derived and Archimedean bead densities.

Suites of multiple fragments of the same meteorite have been studied. These meteorite suites are Sikhote-Alin (8), Gibeon (4), WhiteCourt (8), Odessa (6) and Canyon Diablo (6). Overall, there was some variation in average density between the two methods (Table 3.4). The differences may be caused by the size and shape of the samples. The results presented in Table 3.4 provide insights into the homogeneity of the samples. A coefficient of variation, again following McCausland et al. (2011), is calculated to quantify inter-fragment variability in the same manner that was used to estimate inter-operator variability (Section 3.3.3). Interfragment variability ranges between 1.25 and 3.29\% (Sikhote-Alin (1.99\%), Gibeon (3.29\%), WhiteCourt (1.25\%), Odessa (2.92\%) and Canyon Diablo (2.73\%)) for laser-derived densities. The corresponding figures are slightly higher for Archimedean bead densities, ranging from $1.35-4.13 \%$ (average is 3.01\%). The variation among fragments in these results indicates that 


\begin{tabular}{|c|c|c|c|c|}
\hline Meteorite & Sample & $\begin{array}{l}\text { Average Laser } \\
\text { Density }\left(\mathrm{g} / \mathrm{cm}^{3}\right)\end{array}$ & $\begin{array}{l}\text { Average } \\
\text { Archimedean } \\
\text { Density }\left(\mathrm{g} / \mathrm{cm}^{3}\right)\end{array}$ & $\begin{array}{l}\text { Coeff. } \\
\text { Correlation } \\
\text { (\%) }\end{array}$ \\
\hline Campo de Ceilo & phil1 & 7.43 & 7.26 & 2.38 \\
\hline Canyon Diablo & h34.5604 & 7.61 & 7.43 & -2.46 \\
\hline Canyon Diablo & UWO_005 & 7.64 & 7.44 & -2.65 \\
\hline Canyon Diablo & 303.008 & 7.26 & 7.74 & 6.18 \\
\hline Canyon Diablo & 303.005 & 7.29 & 7.04 & -3.48 \\
\hline Gibeon & $0702-4$ & 7.35 & 7.21 & -1.91 \\
\hline Gibeon & UWO_012 & 7.05 & 6.80 & -3.80 \\
\hline Mundrabilla & PGB_016 & 7.32 & 7.24 & -1.02 \\
\hline Odessa & $1503-1$ & 7.20 & 7.11 & -1.26 \\
\hline Odessa & OD897 & 7.28 & 7.34 & 0.73 \\
\hline Odessa & $\mathrm{H} 10.5$ & 7.10 & 7.19 & 1.27 \\
\hline Odessa & 1503.4 & 6.98 & 7.17 & 2.74 \\
\hline Sikote Alin & T538 & 7.69 & 7.64 & -0.59 \\
\hline Sikote Alin & T425 & 7.93 & 7.91 & -0.30 \\
\hline Sikote Alin & T483 & 7.70 & 7.67 & -0.31 \\
\hline Sikote Alin & T539 & 7.79 & 7.77 & -0.35 \\
\hline Sikote Alin & $\mathrm{T} 487$ & 7.52 & 7.24 & -3.91 \\
\hline Sikote Alin & T540 & 7.77 & 7.60 & -2.29 \\
\hline Sikhote-Alin & DBSA & 7.55 & 7.63 & 1.11 \\
\hline Toluca & T17 & 7.41 & 7.27 & -1.99 \\
\hline Toluca & 1214.001 & 7.00 & 7.14 & 1.93 \\
\hline Whitecourt & $11617 / 221$ & 7.38 & 7.24 & -1.90 \\
\hline Whitecourt & 46 & 7.52 & 7.32 & -2.71 \\
\hline Whitecourt & $11617 / 184$ & 7.49 & 6.95 & -7.78 \\
\hline Whitecourt & 73 & 7.38 & 7.46 & 1.11 \\
\hline Whitecourt & $11617 / 187$ & 7.56 & 7.50 & -0.90 \\
\hline Zagora & T347 & 6.51 & 6.54 & 0.49 \\
\hline Zagora & T350 & 5.88 & 6.01 & 2.17 \\
\hline
\end{tabular}

Table 3.4: A comparison between laser-derived and Archimedean bead densities for the iron meteorites studied in this project 
the samples are largely heterogeneous. This could be due to the presence of silicate or sulfide inclusions, or perhaps due to variable surface oxidation.

\subsection{Densities and iron meteorite classification}

\subsubsection{Iron meteorite classification}

Different classes of ordinary chondrites have been found to possess distinctive, yet overlapping, ranges of density. When density is combined with magnetic susceptibility, the two properties can be a rapid way to classify many stony meteorites (Consolmagno et al., 2008). The laser-derived densities were plotted by class (Figure 3.10) to investigate potential relationships. IAB covers the widest range of densities. IAB is a complex group, with 5 subgroups, 2 of which are represented by the samples studied (IAB-ung, IAB-sLL). The plots for IIAB and IIIAB occupy a fairly narrow range of densities. Perhaps this is distinctive for the group, but a wider range of samples would need to be studied to validate this result. The range observed could be the product of either weathering, or inhomogeneities between samples. Overall, the plot shows that densities from the four classes studied, IAB, IIAB, IIIAB and IVA, are overlapping.

The densities measured for the Sikhote-Alin samples were among the highest in the study. It also has the distinction of being the sole 'fall' sample in the study. This relationship could potentially be related to the amount of weathering this sample has undergone. Sikhote-Alin has undergone very little weathering compared to the other samples. Conversely, the Odessa meteorite is found to possess the lowest densities. It is known to suffer from a form of chemical weathering called lawrencite. The formation of this iron chloride eventually causes the sample to fall apart, and would lower the sample density at the same time. Figure 3.11 plots the age of 


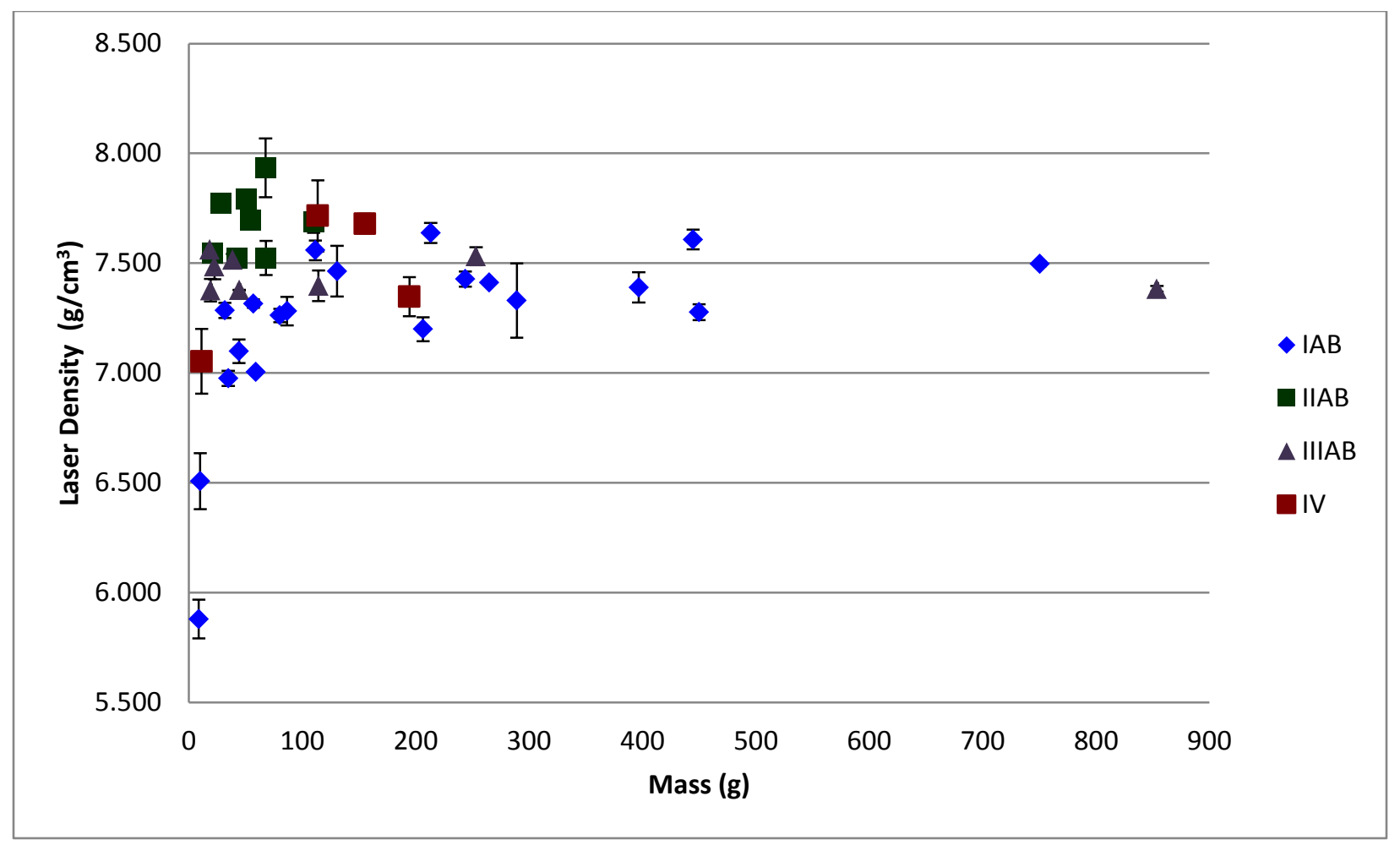

Figure 3.10: Laser density ( 3 operator average) versus mass, and its relation to the chemical classification of iron meteorites. The 2250g Canyon Diablo (\#0303-2) is not included

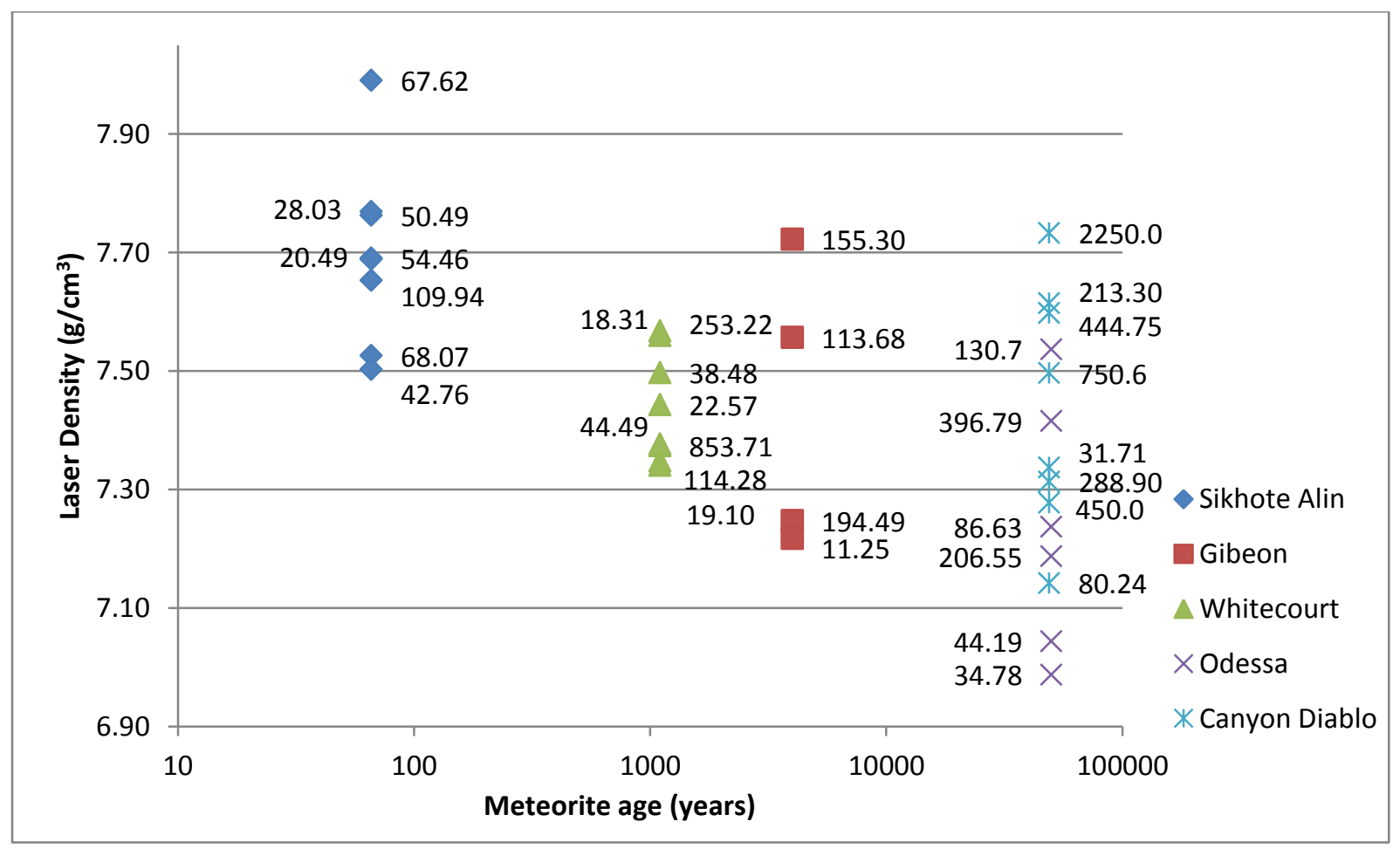

Figure 3.11: Density of the five meteorite suites in relation to the approximate age of their terrestrial arrival. Data labels are sample mass. 
the meteorite suites versus their density. A broad relationship can be seen in which density descends overtime. The oldest samples also possess the greatest range in density. Of note is that the largest sample studied, the $2250 \mathrm{~g}$ Canyon Diablo fragment, possessed a high density similar to that of Sikhote-Alin, suggesting that samples that are multiple kilograms in weight may be less affected by terrestrial weathering, and that their density is more representative of the original material. Likewise, larger samples may be less prone to the density variation caused by sulfide or silicate inclusions. This is not seen in the smaller samples, where there is no relation in size and density.

\subsubsection{Literature Review}

Few density measurements have been published for iron meteorites. As described in Section 1.3.3, some of these date as far back as the mid-1800's (Farrington, 1907). The samples in this study with the most abundant literature density data are Canyon Diablo, Odessa and SikhoteAlin due to their relative abundance in the world's collections. Additionally, densities for Gibeon and Toluca (referred to as "Abert Iron" and "Bethany"), and Campo del Cielo have also been found (Farrington, 1907). A large component

of these data were gathered using destructive methods. The age of the results, pre-1900, suggests that a fluid immersion method may have been used, potentially altering the meteorite and any later results. It is also possible that the samples were also cut into easily measurable geometric shapes to measure their volume. Table 3.5 is a comparison between laser-derived densities obtained in this study and previously published data. Overall, the averaged results of previous studies are somewhat higher than those found in this study. The more recent 
literature bead measurements produced densities that were more consistent with our results.

Older results were almost uniformly higher. The higher densities may be the result of using fluid immersion. The reason for this is difficult to explain, as iron meteorites have very little porosity (Consolmagno and Britt, 1998). It could be that the samples had some permeable feature, such as cracks.

Figure 3.12 is a histogram of all data found in the literature for the IAB, IIAB, IIIAB and IVA groups, the main sources of which were Henderson and Perry (1954) and Farrington (1907), and includes the new data obtained in this study. The study by Henderson and Perry (1954) did not look at the bulk densities of their samples. They cut their meteorites, and selected sections that were free of low density inclusions (such as troilite). Since they focused on meteoritic iron, their results are on the higher end of the histogram. Density values for the different group greatly overlap. As such, Figure 3.12 shows that density alone cannot be used as a classification tool for iron meteorites. 


\begin{tabular}{|c|c|c|c|c|c|c|}
\hline Meteorite & $\begin{array}{l}\text { Laser } \\
\text { Results }\end{array}$ & $\begin{array}{l}\text { Macke, } \\
2010\end{array}$ & $\begin{array}{l}\text { Consolmagno, } \\
1998\end{array}$ & $\begin{array}{l}\text { Lang, } \\
1989\end{array}$ & $\begin{array}{l}\text { Vdovykin, } \\
1973\end{array}$ & $\begin{array}{l}\text { Farrington, } \\
1907\end{array}$ \\
\hline $\begin{array}{l}\text { Campo del } \\
\text { Cielo }\end{array}$ & $\begin{array}{l}7.43 \\
7.56\end{array}$ & & & & & $\begin{array}{l}7.55 \\
7.85 \\
7.77\end{array}$ \\
\hline $\begin{array}{l}\text { Canyon } \\
\text { Diablo }\end{array}$ & $\begin{array}{l}7.61 \\
7.31 \\
7.64 \\
7.26 \\
7.28\end{array}$ & $\begin{array}{l}7.84 \\
7.21\end{array}$ & 7.38 & 7.85 & 7.89 & 7.7 \\
\hline Gibeon & $\begin{array}{l}7.35 \\
7.68 \\
7.72 \\
7.05\end{array}$ & & & & & $\begin{array}{l}7.84 \\
7.84 \\
7.78 \\
7.45\end{array}$ \\
\hline Odessa & $\begin{array}{l}7.39 \\
7.20 \\
7.46 \\
7.28 \\
7.10 \\
6.98\end{array}$ & $\begin{array}{l}7.90 \\
7.01\end{array}$ & 7.15 & 7.831 & & \\
\hline Sikhote-Alin & $\begin{array}{l}7.68 \\
7.52 \\
7.93 \\
7.70 \\
7.79 \\
7.52 \\
7.77 \\
7.55\end{array}$ & $\begin{array}{l}7.61 \\
7.40 \\
7.97 \\
7.66\end{array}$ & & & & \\
\hline Toluca & $\begin{array}{l}7.41 \\
7.00\end{array}$ & & & & & $\begin{array}{l}7.59 \\
7.89 \\
7.72\end{array}$ \\
\hline
\end{tabular}

Table 3.5: Average laser-derived density results in $\mathrm{g} / \mathrm{cm}^{3}$, compared to previously published, and unpublished results. 


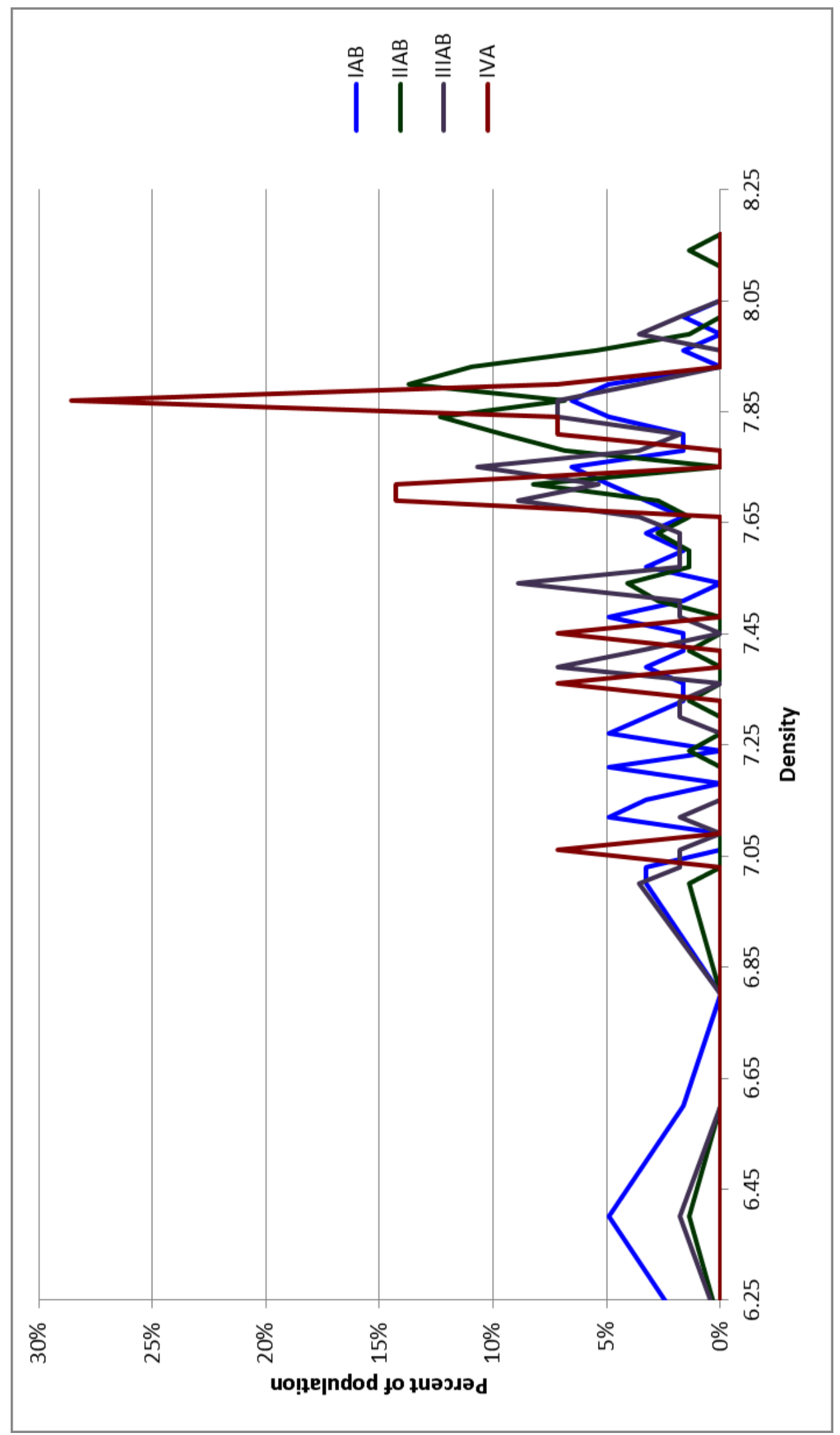

Figure 3.12: Histogram of iron densities in the literature and in this study. 


\section{Tektites}

\section{1: Samples}

A total of 11 tektites were loaned to Carleton University for this project, by Dr. Mel Stauffer, Emeritus Professor, University of Saskatchewan. These splash-form tektites exhibit a variety of shapes and surface textures (Figure 4.1). The samples, originating from the Australasian strewn field, were collected in Thailand or Vietnam. There are multiple examples of teardrops (TT1, TT2, TT3, TT4), ellipsoid-shaped tektites (TO1, TO2, TO3), discs (TD1, TD2) and a single dumbbell sample (TB1) (Table 4.1; Appendix B). Additionally, there is a fragmented tektite with a unique shape (TU1); it has a concave surface that could once have been a large interior vacoule. The surfaces are pitted to varying degrees and have a range of textures, from smooth to appearing frothy with many pits. The pits, caused by surface dissolution (B. Glass, Personal comm.) or degassing (Stauffer and Butler, 2010), can also vary in size. Many of the smaller sized pits (diameter $<2 \mathrm{~mm}$ ) appear glassier than the rest of the sample. The dumbbell tektite TB1, for example, has a smooth surface with few shallow and wide pits (diameter $\sim 5 \mathrm{~mm}$ ). This can be contrasted with the TD1 disk that is covered in twisted schlieren lines, giving the sample a frothy appearance, and the ellipsoid-shaped tektite TO1 that is covered in many small pits (diameter $\approx 2 \mathrm{~mm}$ ). Physically, the samples are all fairly small in size (volume $<75 \mathrm{~cm}^{3}$ ) and are able to fit in the palm of one's hand.

\section{2: Archimedean Bead Measurements}

The tektites proved to lend themselves better to the Archimedean bead method than the iron meteorites (the average error for tektites (average of error listed in rightmost column of Table 4.1 ) is $0.05 \mathrm{~g} / \mathrm{cm}^{3}$ versus $0.58 \mathrm{~g} / \mathrm{cm}^{3}$ for iron (average of error listed in rightmost column 


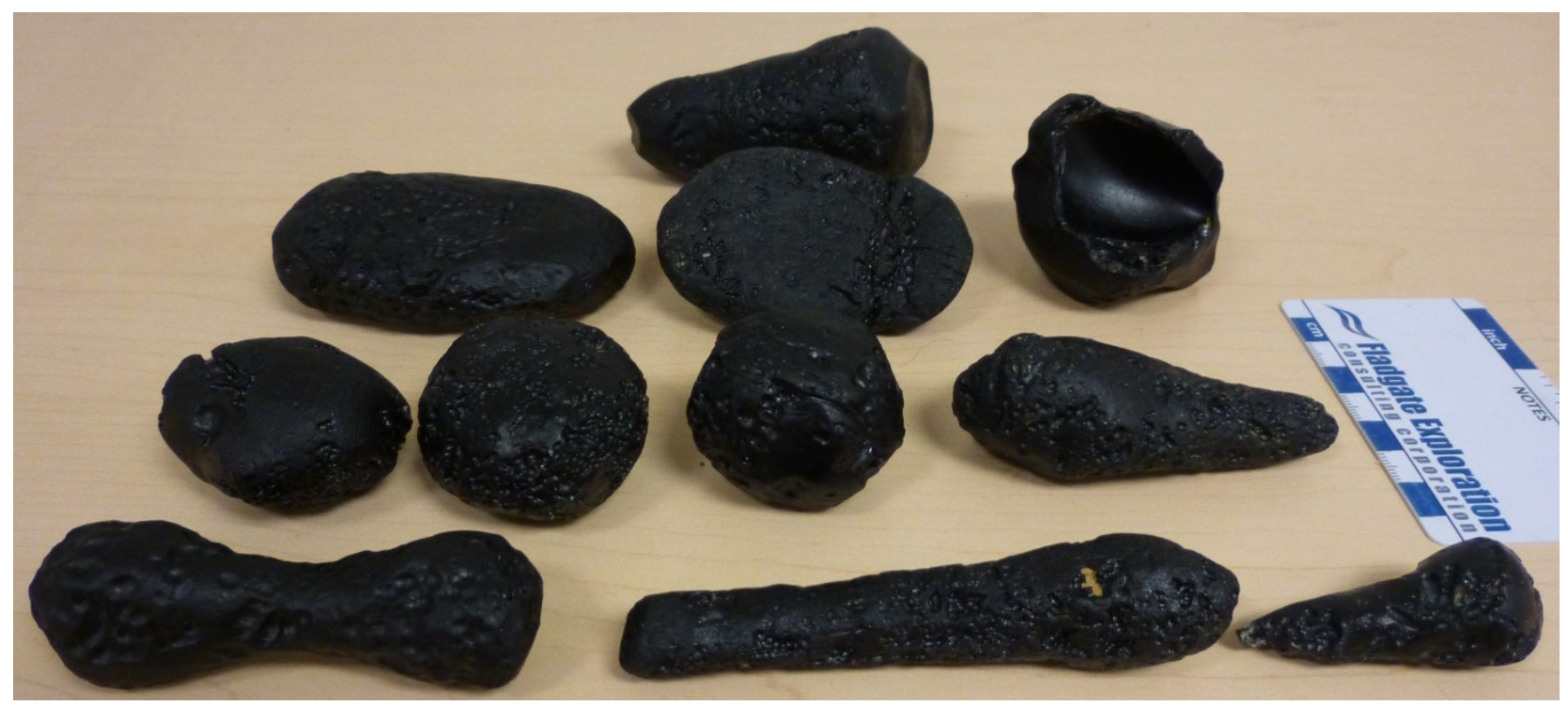

Figure 4.1: The eleven splash-form tektites that were imaged.

\begin{tabular}{|c|c|c|c|c|c|}
\hline \multirow{2}{*}{ Sample } & \multirow{2}{*}{ Shape } & \multirow{2}{*}{ Mass } & \multicolumn{3}{|c|}{ Archimedean bead density } \\
\cline { 3 - 6 } & & & \multicolumn{3}{|c|}{$\left(\mathrm{g} / \mathrm{cm}^{3}\right)$} \\
\cline { 4 - 6 } & & $\mathrm{g})$ & Operator 1 & Operator 2 & Average \\
\hline TT1 & Teardrop & 175.00 & \multicolumn{3}{|c|}{ Sample too large } \\
\cline { 4 - 6 } TT2 & Teardrop & 71.53 & $2.36 \pm 0.04$ & $2.28 \pm 0.04$ & $2.32 \pm 0.04$ \\
\cline { 4 - 6 } TT3 & Teardrop & 53.73 & \multicolumn{3}{|c|}{ Sample too large } \\
TT4 & Teardrop & 21.20 & $2.32 \pm 0.12$ & $2.34 \pm 0.13$ & $2.33 \pm 0.12$ \\
TO1 & Ellipsoid & 78.12 & $2.45 \pm 0.03$ & $2.45 \pm 0.04$ & $2.45 \pm 0.03$ \\
TO2 & Ellipsoid & 68.00 & $2.39 \pm 0.04$ & $2.42 \pm 0.06$ & $2.40 \pm 0.05$ \\
TO3 & Ellipsoid & 59.67 & $2.38 \pm 0.05$ & $2.40 \pm 0.06$ & $2.39 \pm 0.05$ \\
TB1 & Dumbbell & 72.69 & $2.35 \pm 0.04$ & $2.27 \pm 0.04$ & $2.31 \pm 0.04$ \\
TD1 & Disc & 150.00 & $2.35 \pm 0.02$ & $2.32 \pm 0.03$ & $2.33 \pm 0.02$ \\
TD2 & Disc & 84.27 & $2.36 \pm 0.03$ & $2.33 \pm 0.04$ & $2.34 \pm 0.03$ \\
TU1 & Unique & 102.60 & $2.37 \pm 0.03$ & $2.40 \pm 0.04$ & $2.38 \pm 0.03$ \\
\hline
\end{tabular}

Table 4.1: Archimedean bead density measurements, by two operators, of 10 tektite samples. TT: teardrop, TO: ellipsoid, TB: dumbbell, TU: unique shape. 
of Table 3.2). Their low density and moderate size are fairly reminiscent of the stony meteorites for which this method was originally developed (Consolmagno and Britt, 1998). As a result, the tektites will lack the inherent error caused from compaction of the beads by heavy iron meteorites. The procedure remains the same as previously described (Section 2.2.2). Errors can be introduced if there are many pits on the tektite surfaces. Care does need to be taken when placing the tektite in the bead vessel to ensure that the least pitted surface is facing down. This is to reduce the potential effect of air pockets being present during the measurements and affecting the outcome. As an added precaution, the sample was pre-buried before the measurements took place, to allow the beads to cling to the interior of the pits.

Teardrop samples TT1 and TT3 had dimensions that did not allow for their density to be measured with the beads; they were too long to fit into the vessel. For the remaining samples, two operators made 10 repeat measurements each. The densities measured by the two operators are mainly consistent, falling within the tolerance of measurement error (Figure 4.2). The overall average density is $2.36 \pm 0.05 \mathrm{~g} / \mathrm{cm}^{3}$ (Table 4.1 ).

Shape does appear to have had some effect on the results of operator 2 , with different forms clustering at different densities (Figure 4.2). These 'clusters' can be observed above and below a single standard deviation from the mean. The ellipsoid tektites were seen to possess the largest densities out of the samples measured, while the teardrops were at the lower end of the density spectrum. These are preliminary observations to make as only nine samples were studied. 


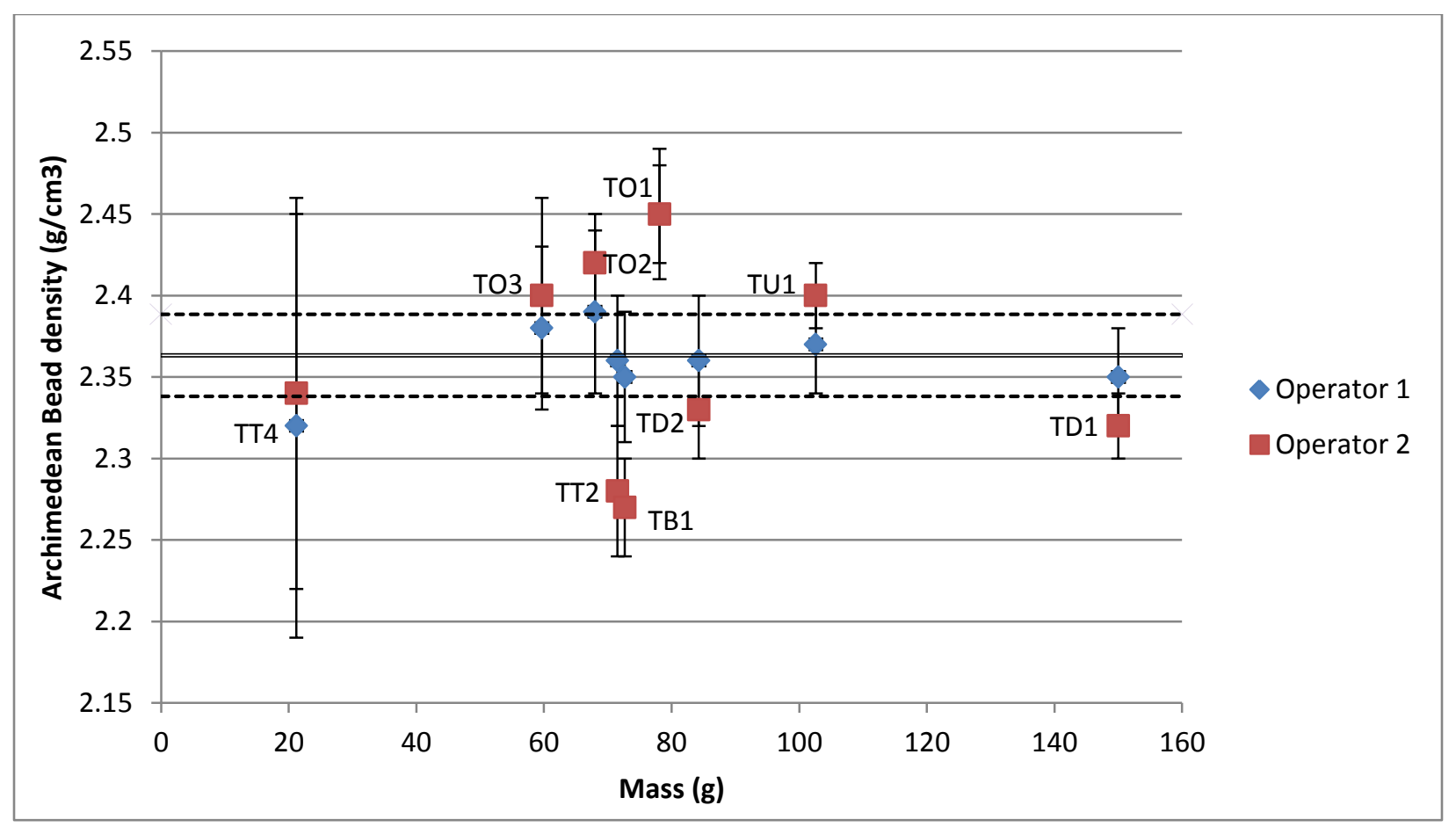

Figure 4.2: Archimedean bead density results of two operators, with the calculated error. The solid line is the average, and the dashed are the first standard deviation

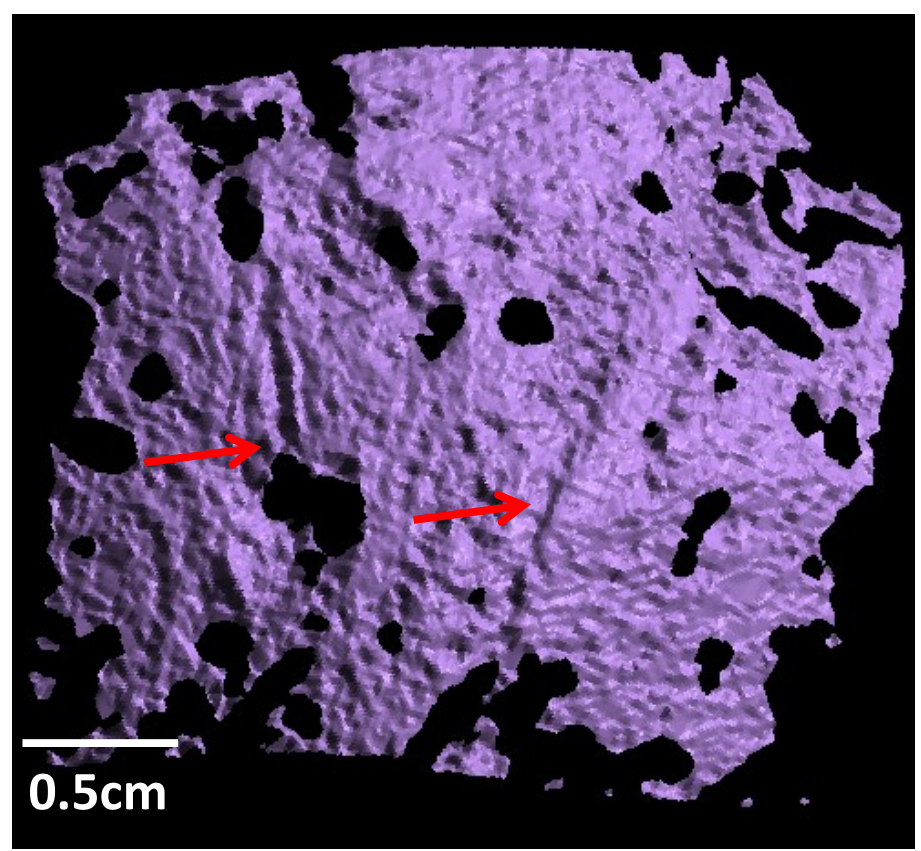

Figure 4.3: Schlieren are seen as traces (indicated by arrows) on the tektite surface on individual images. They do not appear in final models. 


\section{3: Laser imaging method}

\subsection{1: Image Acquisition and Assembly}

Tektites are somewhat unlike any material that has been imaged previously using 3D laser imaging. Three important differences set them apart: (1) material, (2) surface and (3) shape.

Tektites, as mentioned in Chapter 1, are a form of natural glass. Their glassy nature, however, did not pose a challenge to laser imaging. The laser beam did not penetrate the samples and captured surface features with a high fidelity. This might be due to the fact that the surfaces of the tektites studied were matte. This can be contrasted to the more vitreous surface of obsidian.

The tektite surfaces are covered in pits of varying diameters $(1-6 \mathrm{~mm})$ and depths $(1-3 \mathrm{~mm})$. Many of the pits are small and spherical, dissimilar to the 'thumbprint' look of the regmaglypts imaged on the iron meteorites. In most cases, the presence of pits produces a hole in the image data as the projected laser beam is rarely reflected on the bottom of the cavities because of their narrow aperture. As such, this problem is more severe for small and deep pits than for broad and shallow pits. Extensive manual editing was required to fill these holes. The holes in the images were also associated with a number of spurious artifacts and edge distortion. The number of artifacts present made it time consuming to edit each image individually, aside from trimming edges. By comparing multiple overlapping images, spurious protrusions that stood out were highlighted and removed while leaving the rest of the surface intact. Two features observed on the surface of some samples were schlieren, a structure feature and lechatelierite, an inclusion in the glass. Schlieren are thick enough on the surface to be recognized on a number of individual images (Figure 4.3). Their relief may be at the limit of the camera's 


\begin{tabular}{|c|c|c|c|c|c|c|c|c|}
\hline \multirow{2}{*}{ Sample } & \multirow{2}{*}{ Shape } & \multirow{2}{*}{$\begin{array}{c}\text { Mass } \\
\text { (g) }\end{array}$} & \multicolumn{2}{|c|}{ Number of images } & \multirow{2}{*}{$\begin{array}{l}\text { Number } \\
\text { of } \\
\text { polygonal } \\
\text { elements } \\
\text { in model }\end{array}$} & \multicolumn{3}{|c|}{ Laser-derived results } \\
\hline & & & In library & Used & & $\begin{array}{l}\text { Surface } \\
\left(\mathrm{cm}^{2}\right)\end{array}$ & $\begin{array}{c}\text { Volume } \\
\left(\mathrm{cm}^{3}\right)\end{array}$ & $\begin{array}{l}\text { Density } \\
\left(\mathrm{g} / \mathrm{cm}^{3}\right)\end{array}$ \\
\hline TT1 & Teardrop & 175.00 & 58 & 14 & 405950 & 115.16 & 72.02 & 2.43 \\
\hline TT2 & Teardrop & 71.53 & 72 & 15 & 212700 & 67.03 & 30.12 & 2.38 \\
\hline TT4 & Teardrop & 21.20 & 56 & 13 & 90676 & 30.11 & 8.79 & 2.41 \\
\hline TO1 & Ellipsoid & 78.12 & 60 & 28 & 223740 & 66.43 & 33.53 & 2.33 \\
\hline TO2 & Ellipsoid & 68.00 & 56 & 29 & 187442 & 53.31 & 28.24 & 2.41 \\
\hline TO3 & Ellipsoid & 59.67 & 46 & 39 & 217828 & 55.77 & 27.18 & 2.20 \\
\hline TB1 & Dumbbell & 72.69 & 54 & 23 & 183752 & 66.95 & 28.97 & 2.51 \\
\hline TD1 & Disc & 150.00 & 54 & 20 & 418670 & 112.23 & 57.73 & 2.60 \\
\hline TD2 & Disc & 84.27 & 58 & 16 & 318128 & 84.01 & 33.73 & 2.50 \\
\hline TU1 & Unique & 102.60 & 59 & 25 & 290120 & 85.60 & 43.02 & 2.39 \\
\hline
\end{tabular}

Table 4.2: 3D models and laser density. TT3 did not produce viable images for model creation.

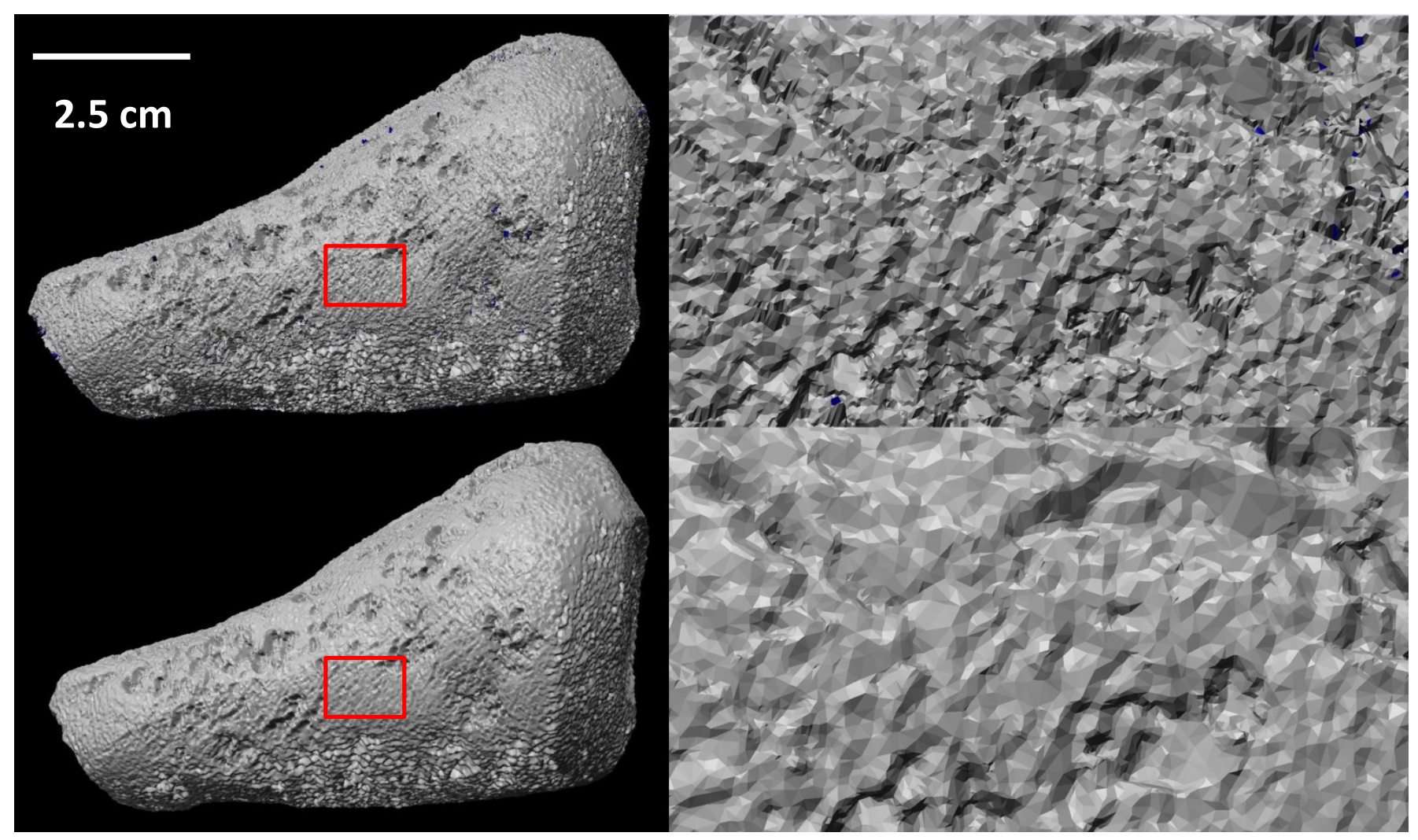

Figure 4.4: 3D polygonal model of tektite TT1. Top: Meshes merged without smoothing. Bottom: Meshes merged with low levels of smoothing. The red box indicates the zoomed area 
accuracy as they do not appear in some images as extensively as on the tektite. Schlieren are lost when individual meshes are merged and excess overlap between them is removed (Section 2.1.2) and when a smoothing function is applied to models to attenuate artifacts (as discussed below). As a result, they do not appear on final 3D models. One sample (TO2), with a fairly smooth surface and more pronounced schlieren may be an exception. Lechatelierite was not observed at all in the images.

Finally, tektites are fairly rounded in shape, some more so than others (e.g. ellipsoid vs disc). The rounded surfaces did not produce a large return, often only encompassing a fraction of the total sample surface. Teardrop TT3 was unable to be modeled because the sample was very thin and pitted, causing each individual image to capture very little of the surface. The images were very limited in extent and indistinct and, as a result, they could not be merged together.

To produce a complete model, anywhere from 13-39 images were used (Table 4.2), many of which were used to fill in holes left by the pits. The more rounded the sample was, the more images were used. The ellipsoid samples, as the most rounded, required the most images. The lack of salient features on the tektite surfaces did pose a challenge during the image assembly process. There were times when it was difficult to accurately find matching points on two separate images. The operator can usually find such points on the tektites by quick visual inspection but, in some areas, there was a lack of anything distinguishable on the surface. As a result, the alignment program had difficulty linking adjacent images together in place, even when the operator manually seeded the software with corresponding points on both images. The simplest way to solve the issues of rounded surfaces and lack of salient points was to use images with a large amount of overlap. 
The 3D polygonal models exhibited a number of small $(<1 \mathrm{~mm})$ artifacts, expressed as small ripples on the surface, once the meshes had been merged (Figure 4.4; top). The ripples covered large swaths of the model's surface, giving it an unrealistic texture. A smoothing function was used to attenuate these artifacts and most remaining spurious protrusions from the model (Figure 4.4; bottom). A low degree of smoothing was applied to all the tektite models. The smoothing function in Polyworks uses a tolerance-based filter. The term "low degree" indicates that points being smoothed are moved to a new position from their original location, based on the average distance of points within a $0.2 \mathrm{~mm}$ radius. While beneficial in removing digitizer noise, and thus many artifacts from the model, this method does unfortunately remove the finer details of the model. Details removed by smoothing include smaller pits, sharp edges and schlieren lines.

Even after smoothing, editing the final 3D polygonal model of the tektites proved to be laborious. The models were not 'watertight'; there were many minute holes that had to be filled in, especially for the ellipsoid samples. Polyworks can perform this task automatically, but it does not always do so correctly. Some of the more complicated holes will produce unrealistic shapes such as large funnels, tubes or spheres. The tektite models had an order of magnitude more holes than the iron meteorites (Figure 4.5) and required anywhere from 2 hours to 15 hours (for the ellipsoid TO1) to examine and edit. An artifact not noticed previously, a twisted surface (Figure 4.6) was very abundant in the tektite models and needed to be corrected manually. This artifact arises where the triangles that make up the polygonal surface have twisted, or overlapped or intersected each other, rather than form a smoothly interlocking mesh. This artifact may be the result of the merging of meshes where the overlap has not been 


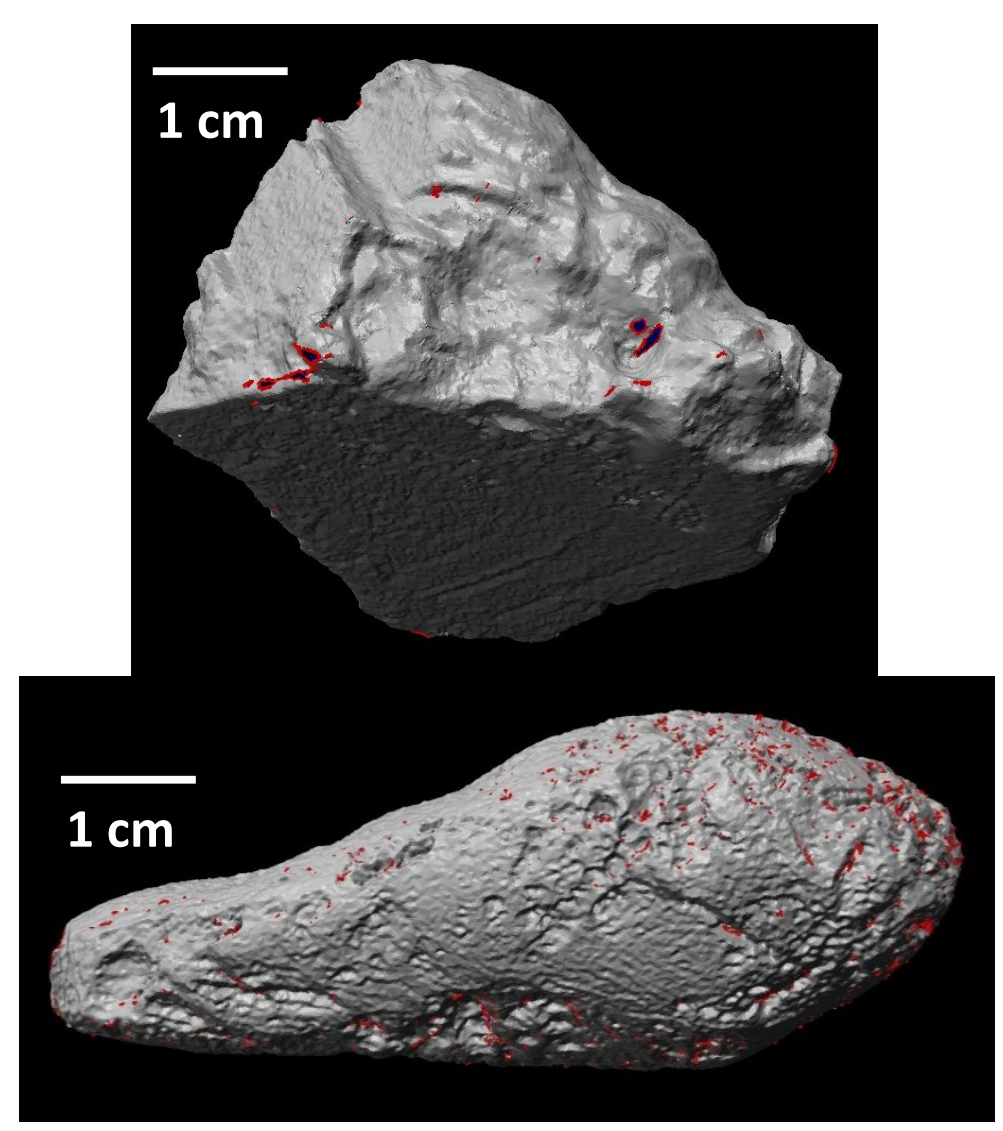

Figure 4.5: Red spots indicate holes in the polygonal surface that need to be filled in order to get a "watertight" final 3D model. These samples are representative of the typical number of holes in tektite and iron meteorite models. Above: 59g Toluca (\#1214.001) fragment. Below: 71.53g teardrop (TT2) tektite.

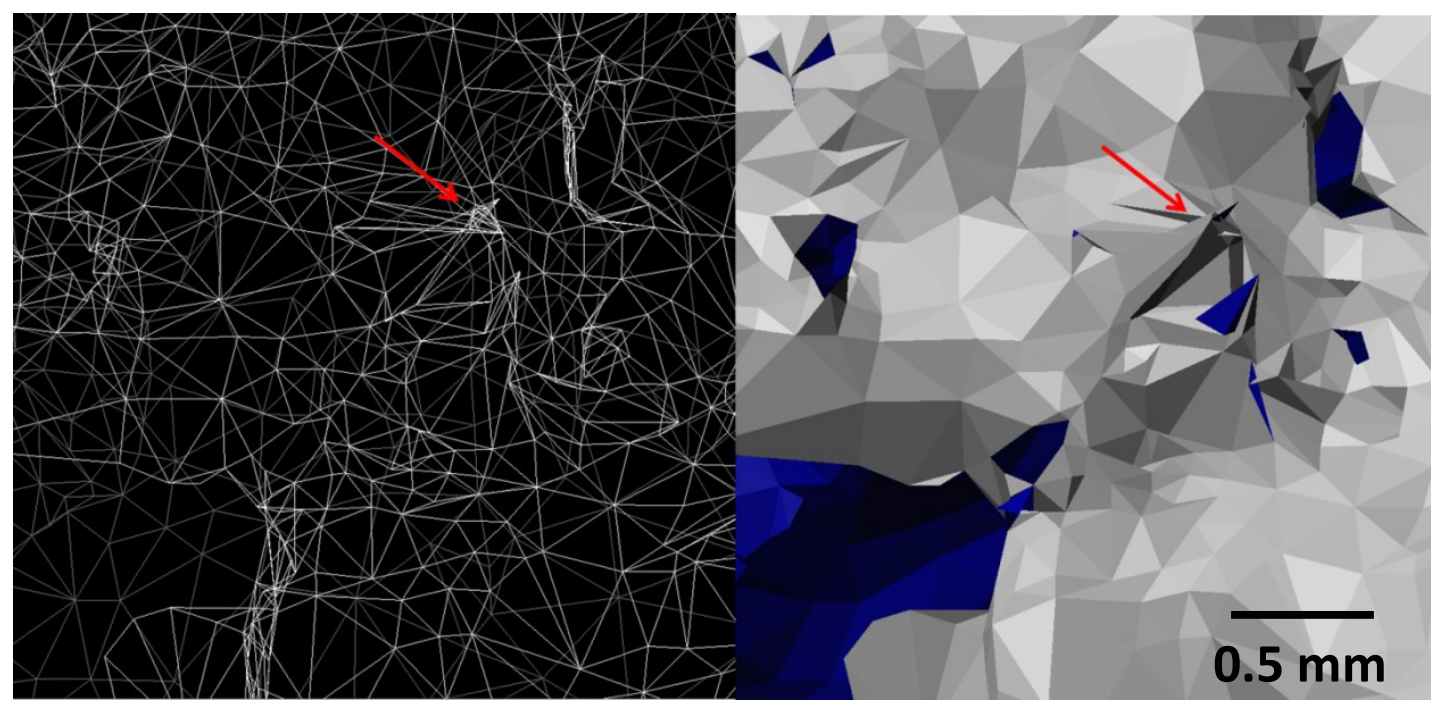

Figure 4.6: The triangles that make up the image have not merged properly, creating an interlocking artifact that appears in the tektite models. The red arrows point to like points on the polygonal mesh (left) and polygonal surface (right). 
properly reduced. This would occur when there is space in between the meshes. This returns to the notion that the lack of salient features causes problems when the meshes are aligned.

\subsection{2: Results}

Table 4.2 details the density results calculated from the 3D models. They range from 2.20 $2.60 \mathrm{~g} / \mathrm{cm}^{3}$ and the average density is $2.41 \pm 0.11 \mathrm{~g} / \mathrm{cm}^{3}$. This is a fairly large variation, especially for samples from the same strewn field. To examine this variation, density was plotted against several metrics representative of the physical size of the sample, such as its volume (Figure 4.7A), surface area (Figure 4.7B) and mass (Figure 4.7C). The sample population is not large enough to draw any solid conclusions. Figure 4.7D explores the relationship between density and shape complexity. Using the surface/spherical equivalent index introduced in section 3.3.3, density can be seen to increase as sample complexity does, which suggests that the challenge of building models of more complicated shapes will produce an underestimation in volume. This trend, however, has not been observed for iron meteorites (Figure 3.7D).

The TD1 disc and TO3 ellipsoid tektites are two obvious outliers, while the rest of the samples mainly cluster close to the $2.40 \mathrm{~g} / \mathrm{cm}^{3}$ average. The TD1 disc tektite, at a density of $2.60 \mathrm{~g} / \mathrm{cm}^{3}$, has one of the more complicated surfaces because of schlieren lines weaving around and many small pits. The schlieren did not appear in the final model. Perhaps their lack of inclusion was enough to cause an underestimation in volume. In addition, there were few salient features along the edges of the TD1 tektite. Another possible cause of the discrepant density value of TD1 tektite therefore could be that the two sides of the disc were fit more closely together during model construction than they should have been. At the other end of the density spectrum $\left(2.20 \mathrm{~g} / \mathrm{cm}^{3}\right)$ is the TO3 ellipsoid tektite. Its surface is smooth on one side and 

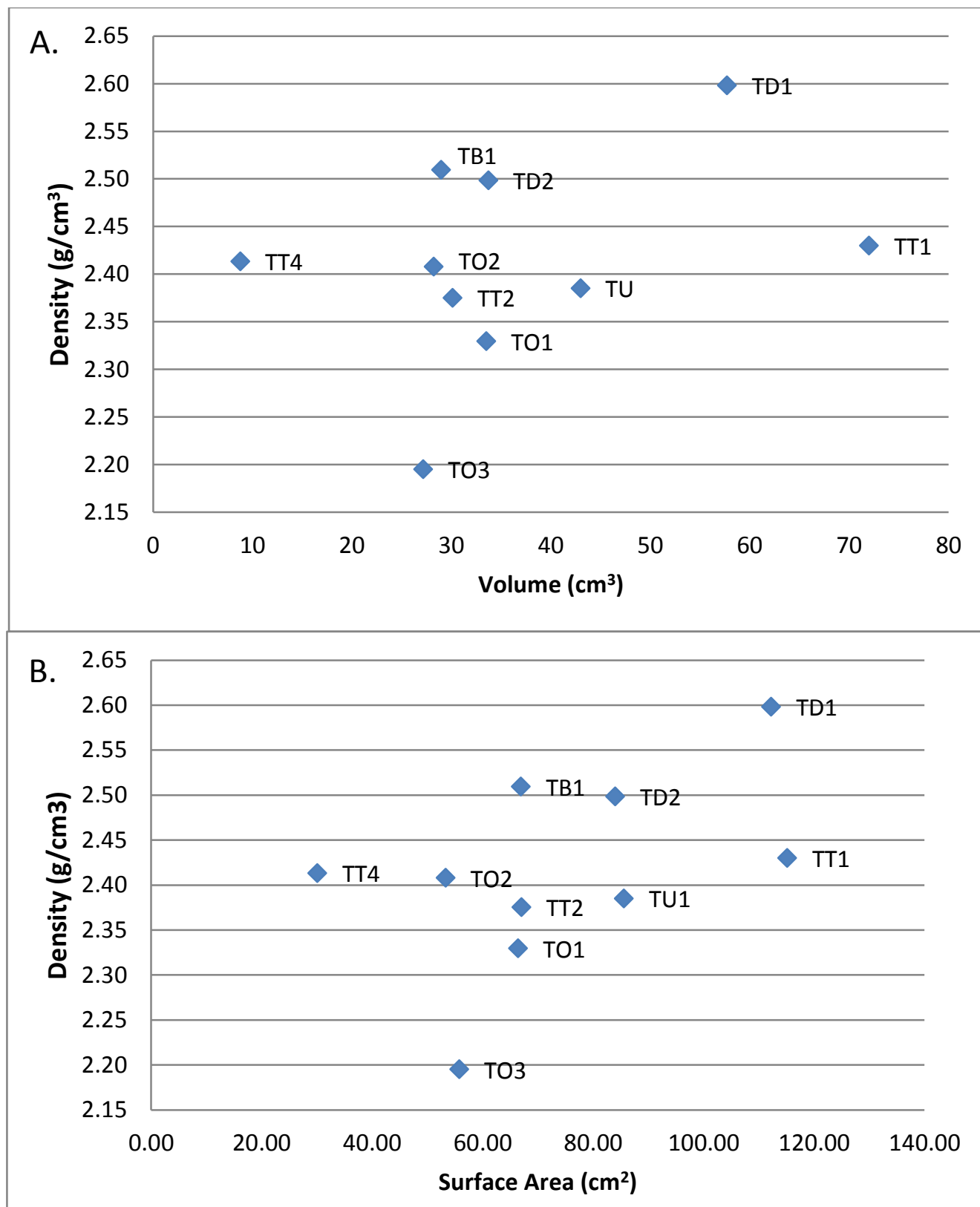

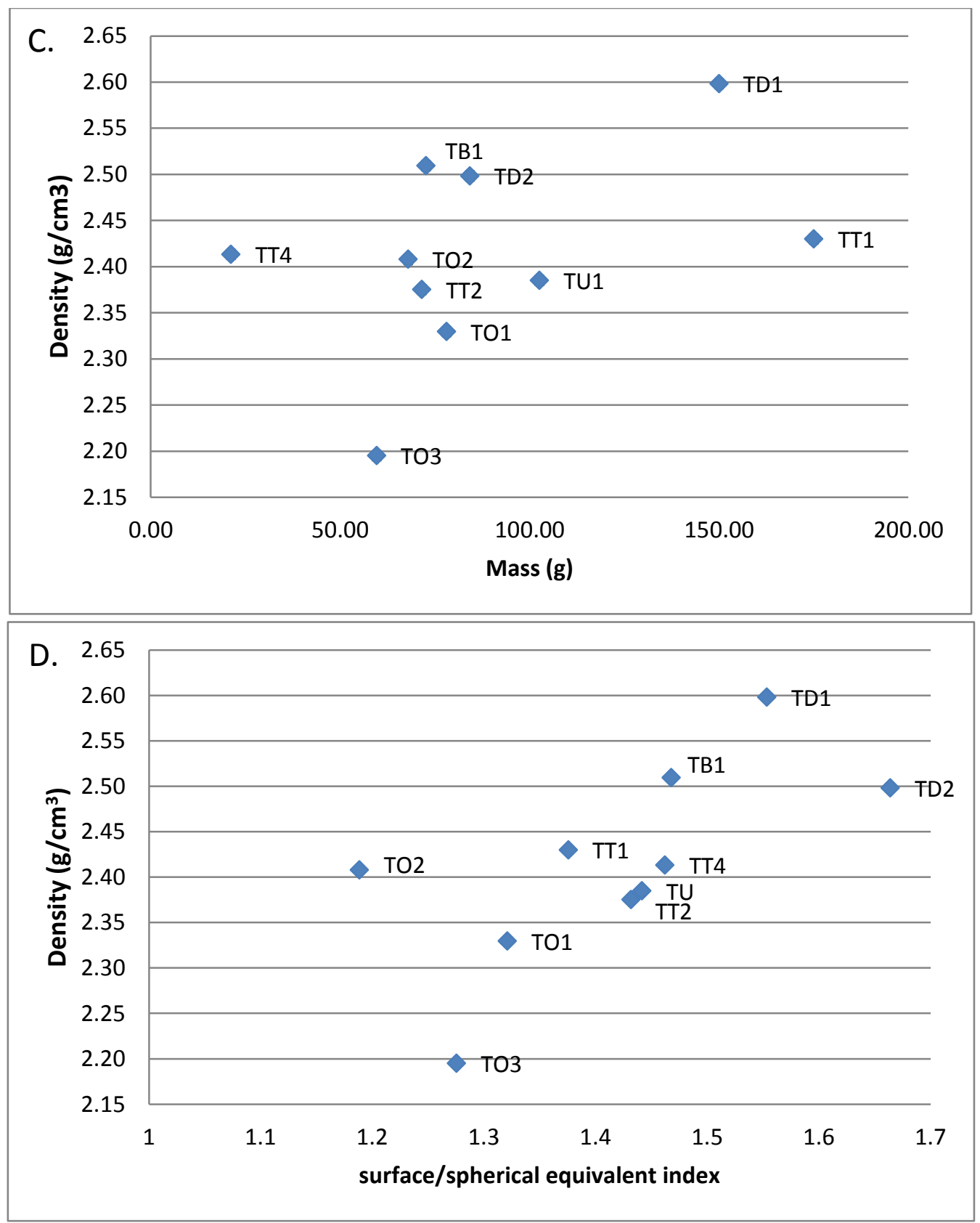

Figure 4.7: A-C: Volume, surface area and mass, representing the size of the sample, are used to examine the cause of the outlying points. D: The surface/spherical equivalent displays surface complexity compared to calculated density. 
pitted on the other, making model assembly problematic. The model likely suffers from artifacts linked with the pits and the lack of salient features. Since the densities determined using 3D laser imaging for TD1 and T03 are outside one standard deviation from the mean (corresponding to a range between $2.30-2.52 \mathrm{~g} / \mathrm{cm}^{3}$ ); these data are considered unreliable. Excluding them from calculations brings the mean density and standard deviation of the remaining tektites to $2.42 \pm 0.06 \mathrm{~g} / \mathrm{cm}^{3}$.

There may be a relationship between the sample density and the shape. This has been noted before by Baker and Forster (1943) but later refuted by Schmude (2002). More samples would need to be examined but, in this study, disc tektites are producing higher densities (average is $2.55 \mathrm{~g} / \mathrm{cm}^{3}$ for 2 samples), teardrops average $2.40 \mathrm{~g} / \mathrm{cm}^{3}$ (3 samples) and ellipsoid samples are trending to lower densities (average is $2.31 \mathrm{~g} / \mathrm{cm}^{3}$ for 3 samples). These densities are from the laser method. Unfortunately, these data contradict the shape relationships observed in the bead results in Figure 4.2, where the ellipsoids produce higher densities, and discs produce lower densities. Any relationship between density and shape remains an open question.

The average coefficient of correlation between the two methods used to calculate density is 3.73\% (using equation 3.2; samples TT1, TD1 and TO3 excluded). This is based on a comparison of 7 samples. The laser method is seen to produce densities that are larger than those determined using the beads. The 1:1 line represents a perfect correlation between the two methods, which does occur twice (TO2, TU1) (Figure 4.8).

There is now a database of 18 stony meteorites (Fry et al., 2013), 28 iron meteorites (this study), and 9 tektites (this study) for which a comparison between the two density 


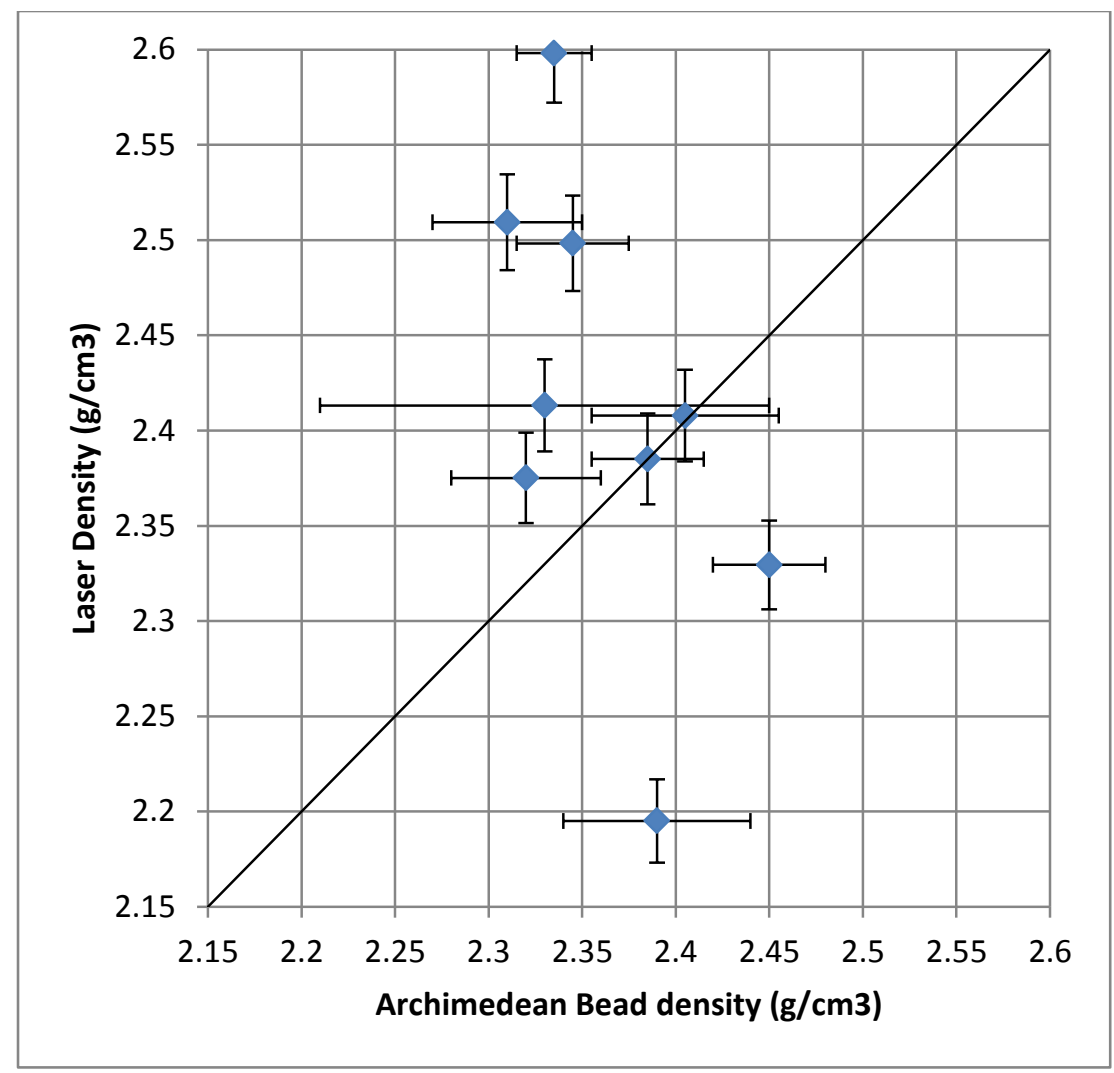

Figure 4.8: Laser derived density versus Archimedean density for 9 tektites, plotted with respect to a 1:1 reference line.
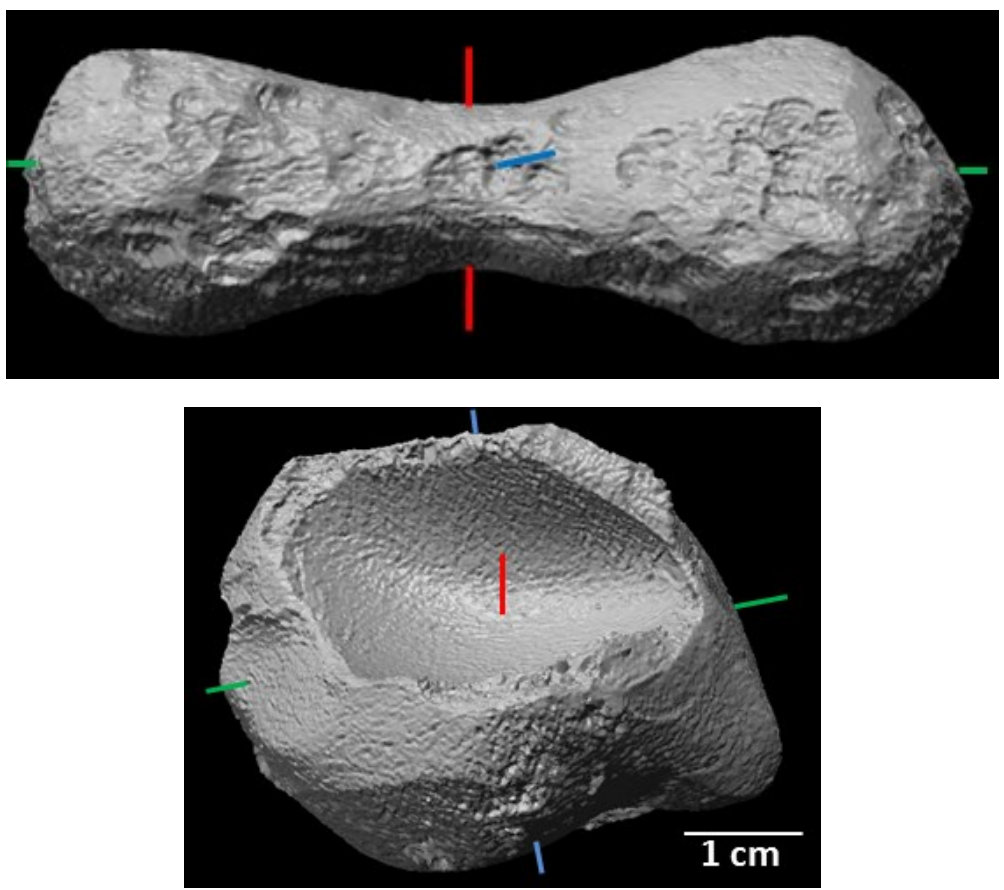

Figure 4.9: Principal rotation axes of (top) 72.68 dumbbell (TB1) and 102.6 unique shape (TU1) are displayed in green (I1), blue (I2) and red (I3). 
measurement methods can be done (in all these cases, the same laser camera (VIVID 9i noncontact 3D digitizer) was used). This includes the results presented in this thesis and those of Fry et al. (2013a).The average coefficient of correlation between both methods is $1.07 \%$ for the stony meteorites and $2.79 \%$ for iron meteorites. For tektites, the coefficient of correlation is much higher, at 3.73\%, even when excluding outliers. The challenges in model building have resulted in the production of a less volumetrically accurate model, which has in turn impacted the accuracy of the density results. From this it is concluded that density measurements of tektites are best done with the Archimedean bead method.

\subsection{3: Aerodynamic Simulations}

Researchers are interested in the mechanics of formation of different tektite shapes.

Different shapes are a function of the axis of rotation and rotation period of the molten ejecta (Stauffer and Butler, 2010). Fry et al. (2013b) used the 3D models built for measuring density to estimate rotation periods. Assuming uniform density, the center of mass of each model was located and the principal moments of inertia for rotation around orthogonal axes through the center of mass were calculated. Eigenvalues and eigenvectors of the inertia tensor were then determined in order to get the three principal moments of inertia (listed in Table 4.3), in increasing order $\left.I_{1}<I_{2}<l_{3}\right)$ and the principal rotation axes.

As well as constraining the rotation rate, the moments of inertia represent the second moment of the mass distribution and therefore serve as first order shape descriptors. The teardrops and dumbbell (Figure 4.9a) all have $I_{1}<<I_{2} \sim I_{3}$ which is indicative of a prolate shape while TO1 and TO2 have $I_{1} \sim I_{2}<<l_{3}$ which is indicative of an oblate shape. TO3, TD1, TD2 and TU1 (Figure 4.9b) are all triaxial shapes with three different moments of inertia. 
Centrifugal forces that result from rotation cause mass to move away from the rotation axis and hence increase the moment of inertia about this axis while the tektites are hot enough to viscously deform. As a result, it is likely that the eigenvector corresponding to the largest moment of inertia (red) corresponds to the rotation axis of the tektite while in flight. Furthermore, there are constraints on the angular momentum needed in order for a rotating fluid blob to achieve a given shape. Using these estimated angular momenta, the rotation period for the tektites is estimated to be of the order of 1s (Butler et al., 2011) (Table 4.3). The rotation rate can only be estimated for tektites that do not show signs of having separated or broken while brittle or in the late stages of their viscous deformation. For this reason teardrop tektites TT1, TT2 and TT4 were not analyzed as they are thought to have formed by overstretch and separation of dumbbells. The analysis is not appropriate either for tektite TU1 whose unique shape features a large broken bubble bit.

Futhermore, the 3D models can be used in numerical simulations of air flow (Figure 4.10) over tektites while in flight to calculate the drag coefficients and the magnitudes of the aerodynamic forces on their surface. Keep in mind that the numerical simulations would only describe the tektites in the moment before it froze, since the shape would have been changing while the tektite remained in a molten state.

\section{4: Comparison with Literature Data}

The laser-derived densities obtained in this study range from $2.20-2.60 \mathrm{~g} / \mathrm{cm}^{3}$ with an average of $2.41 \pm 0.11 \mathrm{~g} / \mathrm{cm}^{3}$ (Table 4.2 ). In this study density was also measured using the Archimedean bead method (Table 4.1 ) which averaged $2.36 \pm 0.05 \mathrm{~g} / \mathrm{cm}^{3}$. The laser-derived and 
Archimedean densities are in broad accord with early measurements of the densities of Australasian tektites by water immersion (Baker and Forster, 1943) and zinc salt flotation (Chapman et al., 1964), and more recent data also acquired by water immersion (Schmude, 2002), which fall in the range $2.40-2.45 \mathrm{~g} / \mathrm{cm}^{3}$. They are also consistent with specific gravity data of micro tektites (usually less than $1 \mathrm{~mm}$ in diameter) from Australia, which range from 2.37-2.55 g/ $/ \mathrm{cm}^{3}$ (Cassidy et al., 1969). The results from the present study are slightly lower than previously published data. The fluid used in immersion measurements might have invaded pores and affected the results. Fluid invasion, however, would cause the volume of the fluid to become larger and the density to be underestimated, which is the opposite of the trend observed. The difference between the densities measured in this study and previous published data therefore remains an issue not fully resolved. Of note is that the sample population of tektites considered in this study is very small compared to the several hundred specimens analyzed by other researchers. 


\begin{tabular}{|c|c|c|c|c|c|c|c|c|c|}
\hline \multirow{3}{*}{ Sample } & \multirow{3}{*}{ Shape } & \multirow{3}{*}{$\begin{array}{l}\text { Mass } \\
\text { (g) }\end{array}$} & \multicolumn{3}{|c|}{ Dimensions } & \multicolumn{3}{|c|}{$\begin{array}{c}\text { Normalized moment } \\
\text { of inertia }\end{array}$} & \multirow{3}{*}{$\begin{array}{c}\begin{array}{c}\text { Rotation } \\
\text { period }\end{array} \\
\\
\text { (s) }\end{array}$} \\
\hline & & & \multirow{2}{*}{$\begin{array}{c}\text { Length } \\
(\mathrm{cm})\end{array}$} & \multirow{2}{*}{$\begin{array}{l}\text { Width } \\
(\mathrm{cm})\end{array}$} & \multirow{2}{*}{$\begin{array}{c}\text { Thickness } \\
(\mathrm{cm})\end{array}$} & \multicolumn{3}{|c|}{-} & \\
\hline & & & & & & $I_{1}$ & $\mathrm{I}_{2}$ & $\mathrm{I}_{3}$ & \\
\hline TT1 & Teardrop & 175.00 & 8.71 & 4.81 & 4.35 & 0.26 & 0.74 & 0.76 & - \\
\hline TT2 & Teardrop & 71.53 & 8.30 & 3.33 & 2.80 & 0.21 & 0.97 & 1.01 & - \\
\hline TT4 & Teardrop & 21.20 & 5.66 & 2.32 & 2.13 & 0.22 & 1.01 & 1.03 & - \\
\hline TO1 & Ellipsoid & 78.12 & 4.78 & 4.59 & 3.15 & 0.36 & 0.40 & 0.52 & $0.96-1.52$ \\
\hline TO2 & Ellipsoid & 68.00 & 4.38 & 4.02 & 3.48 & 0.37 & 0.40 & 0.47 & $0.78-1.23$ \\
\hline TO3 & Ellipsoid & 59.67 & 4.76 & 3.92 & 3.10 & 0.33 & 0.45 & 0.51 & $0.84-1.32$ \\
\hline TB1 & Dumbbell & 72.69 & 8.42 & 2.87 & 2.83 & 0.18 & 1.69 & 1.70 & $1.45-2.29$ \\
\hline TD1 & Disc & 150.00 & 8.70 & 5.32 & 2.68 & 0.29 & 0.75 & 0.93 & $1.30-2.00$ \\
\hline TD2 & Disc & 84.27 & 7.55 & 5.00 & 1.79 & 0.35 & 0.91 & 1.19 & $0.93-1.47$ \\
\hline TU1 & Unique & 102.60 & 5.74 & 4.64 & 4.15 & 0.39 & 0.49 & 0.61 & - \\
\hline
\end{tabular}

Table 4.3: Moments of inertia and rotation period of tektite samples 


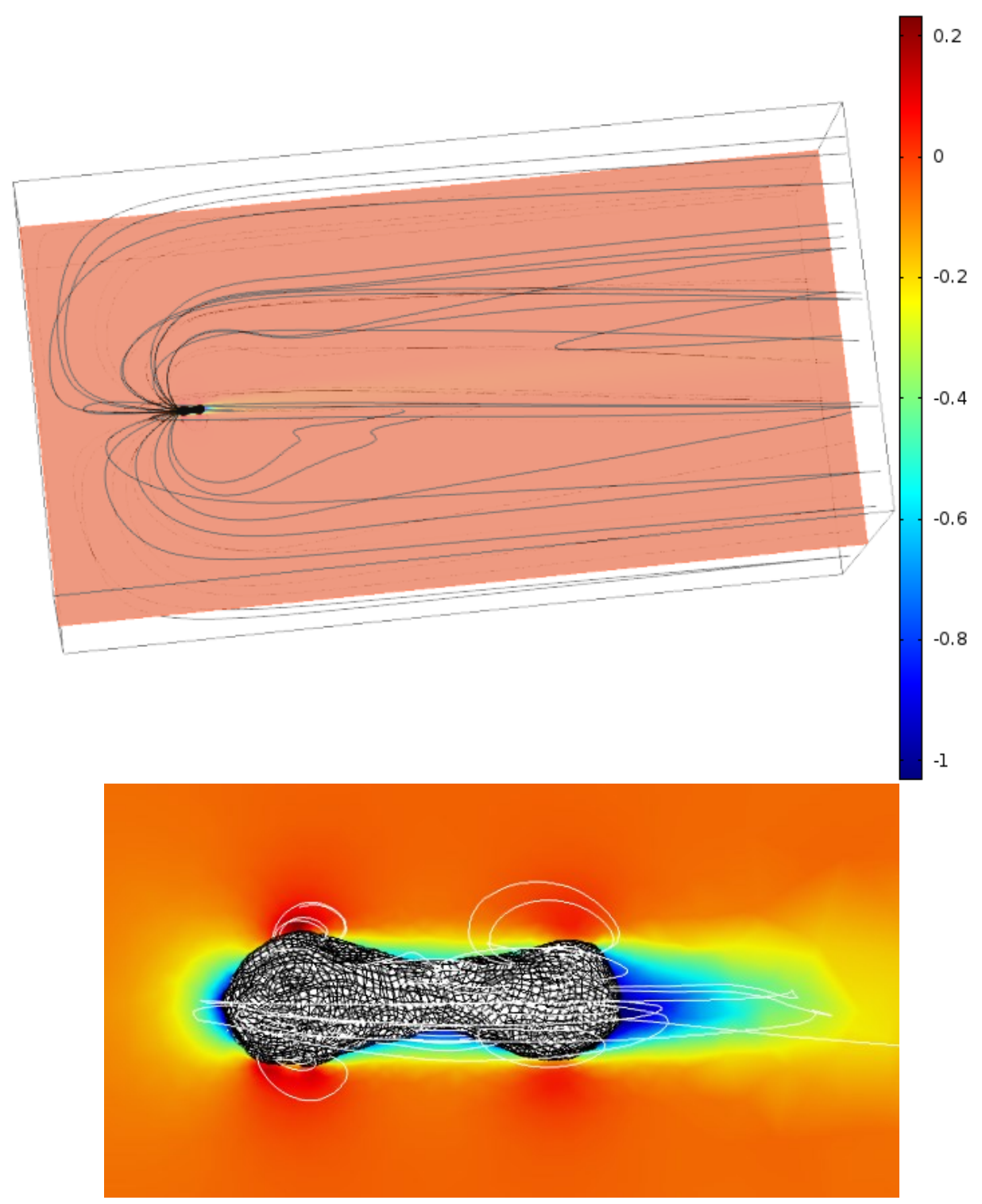

Figure 4.10: The results of a simulation of air flow over the model of the dumbbell (TB1) tektite for a Reynolds number of 20,000. The flow direction is from left to right. Air enters the box from the left at a constant dimensionless horizontal velocity of 1 . A constant value of 1 was subtracted from the results in order to show the fluid velocity as if the tektite were moving through still air with a velocity of -1. Colors indicate the magnitude of the air velocity and vary from 0 (blue) to 1 (red). Top: General view; Bottom: zoomed view. 


\section{Summary, Conclusions and Future Work}

This study focused on studying iron meteorites and tektites. While these two objects are quite dissimilar, the common goal of the project was to capture their surface features and shape in 3D. Overall, 41 iron meteorite fragments and 10 tektites were imaged using a VIVID 9i laser camera, produced by Konica Minolta. Individual images were used to create highly detailed and volumetrically accurate models. The bulk density of each sample was calculated from the models. The Archimedean bead method was used to provide a comparison to these new results.

There were few major challenges in imaging iron meteorites. The camera was able to image a range of sizes, from $1 \mathrm{~cm}$ to $15 \mathrm{~cm}$ (about 3 orders of magnitude in mass), as well as a variety of shapes, from blocky to shrapnel like. As well, it was able to image curatorial features such as stickers and labels, to a degree in which they were legible on the final model. The camera did have difficulty with cut faces, when they were presented at an angle to the laser and with sharp edges, though they were overcome by acquiring an extensive library of images. The ability of the camera to image such a wide variety of features attests to its versatility as a laboratory imaging instrument. The laser produced a number of minor artifacts in the images, however these are very easily removed by manual editing.

Multiple fragments from several different iron meteorites were studied. These suites, as well as several other samples, belong to four of the most abundant chemical groupings of iron meteorites. This allowed for density comparisons on a number of levels; between fragments of the same meteorite, and between different classes of meteorites. The results showed that the densities between fragments of suites were $2.7 \%$ different on average (the inter-operator variability of laser-derived density is estimated to be $0.93 \%$ (Table 3.3), indicating that the meteorites are mostly homogeneous. Plotting the results for all samples showed that density cannot be used to distinguish classes of iron meteorites from one another; the values are overlapping. As the classes are based largely on trace element composition, this is not 
surprising. It might, however, provide an indication as to the amount of terrestrial weathering that the sample has suffered. The Sikhote-Alin suite, the sole fall investigated, produced the highest densities, while the other samples, all finds, were noticeably lower. On the same note, the largest sample studied produced a density comparable to that of Sikhote-Alin, suggesting that larger samples will produce values that are less affected by oxidation minerals.

Consolmagno et al. (2013) are currently investigating the combined use of density and heat capacity to determine the amounts of nickel and sulfides or weathering products in iron meteorites.

The results compared to the Archimedean bead method showed that the laser method, while more time consuming, produces more accurate results. Measurements by different operators are more consistent. Densities calculated from the Archimedean method were found to vary between operator and date. This is likely due to the fact that iron meteorites are dense objects and can cause the beads to undergo differing amounts of compaction within the measurement vessel.

Tektites did not prove as simple to image as iron meteorites. The glassy surface of the tektites did not prove an issue, as originally feared, however, their surface morphology did. The rounded shapes and large number of tiny cupules lead to highly incomplete images, and the lack of salient features caused difficulty in aligning the images. Model completion was more laborious and time consuming as a result. In many cases, the laser did not capture the entirety of the pits, and the remainder was added after. This could explain the variation in density between the samples observed (density ranges from $2.20-2.60 \mathrm{~g} / \mathrm{cm}^{3}$ ), as there are some obvious outliers. Past studies have shown that tektites are fairly homogenous, though there are some inclusions, often $\mathrm{SiO}_{2}$ glass, so some of the laser-derived densities seem in error. In comparison, the Archimedean bead method provided data that indicate fairly homogenous objects (density ranges from $2.31-2.45 \mathrm{~g} / \mathrm{cm}^{3}$ ). 
There are other specialized uses for 3D models of tektites. They can be used by physicists that are interested in the origin of tektite morphology. There has been considerable debate into the origin of tektites and their features. One of the remaining questions is the formation of different tektite shapes, which form as they are solidifying while airborne. The models can be imported into specialized software that measures parameters such as principal moments of inertia around orthogonal axes and from those, rotation period. The models can then be used to run simulations of aerodynamic forces acting on the tektite in flight. Calculations of air flow over the model surface and the resulting drag coefficient can help bring further understanding to the behavior of tektites in flight.

Like any study, there is still much work that could be done. The 3D laser method has proven that it can be useful in investigating an expanding range of materials. Aside from providing a non-destructive method of measuring density, 3D models can be of benefit to collection curators. Keeping a digital model delivers an archival document, should researchers alter the shape of the fragment. Using recent advances in 3D printing, replicas could even be produced. A step to take would be to investigate the accuracy of 3D prints, as applied to meteorite surfaces. Are they high fidelity replicas, or are some of the more discrete features lost in printing.

A degree of automation could be introduced to image acquisition, removing the need for an operator to manually manage the scanning process, cutting the time required by a sizeable fraction. Other potential matters to be addressed are to expand the database of meteorites in this form of study. Nine iron meteorites, out of approximately 1000that are known, were looked at, most of which were from the IAB group. Logically expanding the number of separate meteorites examined is the next step in this study. A benefit of laser imaging as a method of density determination is that it can easily be used on large samples that would otherwise be 
impractical or difficult to measure. It would be of interest to scan larger fragments in the future, and determine an upper limit, if there is one, of sample sizes.

Conversely, the Archimedean bead method is the better choice for measuring the density of tektites. Most density studies conducted to this point have investigated in detail the Australasian field. Applying this methodology to the other strewn fields could be useful. Likewise, applying laser imaging to Muong Nong and ablated form tektites could provide an interesting comparison to the splash-forms studied here. The best avenue of investigation using tektite models at this time are the flow simulations. Aerodynamic drag is likely to significantly affect the downward trajectory of tektites and therefore the final distribution of tektite strewn fields. Perhaps applying flow simulations to a number of tektites from across Australia can be used to help pin point the original crater that produced the field. 


\section{References}

Adams, E.W. and Huffaker, R.M. 1964. Aerodynamic Analysis of the Tektite Problem. Geochimica et Cosmochimica Acta 28: 881-892.

Baker, G. and Forster, H.C. 1943. The specific gravity relationships of australites. American Journal of Science 241:377-406.

Barnes, V.E. 1967. Tektites. International Journal of Geophysics 2: 1507-1518.

Britt, D.T. and Consolmagno, G.J. 2003. Stony meteorites porosities and densities: A review of the data through 2001. Meteoritics \& Planetary Science 38: 1161-1180.

Buchwald, V.F. 1975. Handbook of iron meteorites: Their history, distribution, composition and structure, Volume 1. The University of California Press, Los Angeles. 243pp.

Burbine, T. H., McCoy, T. J., Meibom, A., Gladman, B., \& Keil, K. 2002. Meteoritic parent bodies: Their number and identification. Asteroids III: 653-667.

Butler, S.L., Stauffer, M.R., Sinha, G., Lilly, A., and Spiteri, R.J. 2011. The shape distribution of splash-form tektites predicted by numerical simulations of rotating fluid drops. Journal of Fluid Mechanics 667: 358-368.

Carmichael, R.S. 1989. Practical Handbook of Physical Properties of Rocks and Minerals. CRC Press Inc, Boca Raton, Florida. 741pp.

Cassidy, W.A., Glass, B. and Heezen, B.C. 1969. Physical and chemical properties of Australasian microtektites. Journal of Geophysical Research 75: 1008-1025.

Chapman, D.R., Larson, H.K., and Scheiber, L.C. 1964. Population polygons of tektite specific gravity for various localities in Australasia. Geochimica et Cosmochimica Acta 28: 821-839

Consolmagno, G.J., and Britt, D.T. 1998a. The density and porosity of meteorites from the Vatican collection. Meteoritics \& Planetary Science 33: 1231- 1241.

Consolmagno, G.J., Britt, D.T. and Stoll, C.P. 1998b. The porosities of ordinary chondrites: Models and interpretation. Meteoritics \& Planetary Science 33: 1221-1229.

Consolmagno, G.J., Britt, D.T., and Macke, R.J. 2008. The significance of meteorite density and porosity. Chemie der Erde 68:1-29.

Consolmagno, G.J., Schaefer, M.W., Schaefer, B.E., Britt, D.T., Macke, R.J., Nolan, M.C.,and Howell, E.S. 2013. The measurement of meteorite heat capacity at low temperatures using liquid nitrogen vaporization. Accepted for publication in Planetary and Space Science, doi:10.1016/j.pss.2013.07.009. 
Dressler, B.O. and Reimold, W.U. 2001. Terrestrial impact melts and glasses. Earth-Science Reviews 56:205-284.

Farrington, O.C. 1907. Analyses of iron meteorites, compiled and classified. Field Columbian Museum Publications 120, Geological Series 3, No. 5: 59-110.

Faul, H. 1966. Tektites are Terrestrial. Science 152: 1341-1345.

Fry, C., Samson. C., McCausland, P.J.A., and Herd, R.K. 2012. 3D laser imaging of iron meteorites. (abstract 2703) $43^{\text {rd }}$ Lunar and Planetary Science Conference.

Fry, C., Melanson, D., Samson, C., McCausland, P.J.A., Herd, R.K.,Ernst, R.E., Umoh, J., and Holdsworth, D.W. 2013a. Physical characterization of a suite of Buzzard Coulee H4 chondrite fragments. Meteoritics \& Planetary Science 48: 1060-1073.

Fry, C., Samson. C., Butler, S., McCausland, P.J.A., and Herd, R.K. 2013b. 3D laser imaging of tektites. (abstract 2597) $44^{\text {th }}$ Lunar and Planetary Science Conference.

Flynn, G.J., Moore, L.B., Klöck, W. 1999. Density and porosity of stone meteorites: Implications for the density, porosity, cratering and collisional disruption of asteroids. Icarus 142: 97-105.

Gentner, W., Storzer, D., and Wagner, G.A. 1969. New Fission Track Ages of Tektites and Related Glasses. Geochimica et Cosmochimica Acta 33; 1075-1081.

Glass, B. 1974. Microtektite Surface Sculpturing. Geological Society of America Bulletin 85: 1305-1314.

Glass, B. 1990. Tektites and microtektites: Key facts and inferences. Tectonophysics 171: 393404.

Glass, B. and Koeberl, C. 2006. Australasian microtektites and associated impact ejecta in the South China Sea and the Middle Pleistocene supereruption of Toba. Meteoritics \& Planetary Science 41: 305-326.

Goldstein, J.I., Scott, E.R.D and Chabot, N.L. 2009. Iron meteorites: Crystallization, thermal history, parent bodies and origin. Chemie der Erde 69: 293-325.

Hartmann, W.K., Quantin, C. and Mangold, N. 2007. Possible long-term decline in impact rates: 2. Lunar impact-melt data regarding impact history. Icarus 186: 11-23.

Henderson, E.P. and Perry, S.H. 1954. A discussion of the densities of iron meteorites. Geochimica et Cosmochimica Acta 6: 221-240.

Herd, R.K., Miller, S.J, and Cristie, I. 2003. Measurement of a meteorite volume using a noncontact 3D laser scanner. (abstract 5285) $66^{\text {th }}$ Annual Meteoritical Society Meeting. 
Hewins, R.H. 1997. Chondrules. Annual Review of Earth and Planetary Sciences 25: 61-83.

Hutchinson, R. 2004. Meteorites: A petrologic, chemical and isotopic synthesis. Cambridge University Press, Cambridge, UK. 506pp.

Kohout, T., Kletetschka, G., Elbra, T., Adachi, T., Mikula, V., Pesonen, L.J., Schnabl, P. and Slechta, S., 2008. Physical properties of meteorites - applications in space missions to asteroids. Meteoritics \& Planetary Science 43: 1009-1020.

Konica Minolta. 2006. Non-Contact 3D Digitizer VIVID 9i/VI-9i. Instruction Manual (Hardware). Konica Minolta Sensing Inc.

Koeberl, C. 1990. The geochemistry of tektites: an overview. Tectonophysics 171: 405-422.

Koeberl, C. 1994. Tektite origin by hypervelocity asteroidal orcometary impact: target rocks, source craters, and mechanisms. IN: Dressler,B.O. Grieve, R.A., and Sharpton, V.L. (eds.). Large Meteorite Impacts and Planetary Evolution. Geological Society of America, Spec. Publ., vol. 293. Boulder, Colorado, USA, pp. 133-152.

Lang, B. and Franaszczuk, K. 1989. Fracture cascade at atmospheric entry-Canyon Diablo, Odessa, and Wolf Creek irons. Lunar and Planetary Science Conference Proceedings 19: 683689.

Macke, R.J., Britt, D.T., and Consolmagno, G.J. 2010. Analysis of the systematic error in 'bead method' measurements of meteorite bulk volume and density. Planetary and Space Science 58:421-426

Mah, J., Samson, C., McKinnon, S.D., and Thibodeau, D. 2013. 3D laser imaging for surface roughness analysis. International Journal of Rock Mechanics \& Mining Sciences 58: 111-117.

McCall, G.J.H. 2005. Tektites. IN: Selley, R., Cocks, L.R., and Plimer, I. (eds.). Encyclopedia of Geology. Elsevier Scientific Publishing Company, Amsterdam, Oxford, New York, pp.443-455.

McCausland, P.J.A., Samson, C., and McLeod, T. 2011. Determination of bulk density for small meteorite fragments via visible light 3D laser imaging. Meteoritics \&Planetary Science 46: 10971109.

McSween, H.Y. Jr. 1999. Meteorites and their parent planets, Second edition. Cambridge University Press, Cambridge, UK. 354pp.

Norton, O.R. 2002. The Cambridge Encyclopedia of meteorites. Cambridge University Press, Cambridge, UK. 310pp.

O’Keefe, J.A. 1967. Tektite Sculpturing. Geochimica et Cosmochimica Acta 31; 1931-1933. 
O'Keefe, J.A. 1976. Tektites and their origin. Elsevier Scientific Publishing Company, Amsterdam, Oxford, New York. 254pp.

O’Keefe, J.A. 1994. Origin of tektites. Meteoritics 29: 73-78.

Olson, L. 2013. The 3D imaging of drill core for fracture mapping. M.Sc. Thesis, Carleton University, Ottawa, Ontario.

Olhoeft, G.R. and Johnson, G.R. 1989. Densities of rocks and minerals. In: Carmichael, R.S. (ed.) Practical Handbook of physical properties of rocks and minerals. CRC Press Inc., Florida, pp.139176.

Ralchenko, M. 2013. Physical characterization of the frozen fragments of the Tagish Lake meteorite. B.Sc. Thesis, Carleton University, Ottawa, Ontario.

Rochette, P., Sagnotti, L.,Bourout-Denise, M., Consolmagno, G., Folco, L., Gattacceca, J., Osete, M.L. and Pesonen, L. 2003. Magnetic classification of stony meteorites: 1 . Ordinary chondrites. Meteoritics \& Planetary Science 38 (2): 251-268.

Roy, S.K. and Wyant, R.K. 1949. The Navajo meteorite. Field Museum of Natural History, Geological Series 7, No.8: 113-127.

Roy, S.K. and Wyant, R.K. 1950. The Smithsonia meteorite. Field Museum of Natural History, Geological Series 7, No.9: 129-134.

Schmude, R.W. 2002. Specific gravities of tektites from Guandong, China. Georgia Journal of Science 60: 116-126.

Schön, J.H. 2011. Physical properties of rocks: A workbook. Elsevier Scientific Publishing Company, Amsterdam, Oxford, New York. 481pp.

Smith, D., Ernst, R., Samson, C. and Herd, R. 2006a. Stony meteorite characterization by non-destructive measurement of magnetic properties. Meteoritics \& Planetary Science 41 (3): 355-373

Smith D. L., Samson C., Herd R., DesLauriers A., Sink J.E., Christie I., and Ernst R. E. 2006b. Measuring the bulk density of meteorites non-destructively using three dimensional laser imaging. Journal of Geophysical Research 111:E10002,doi:10.1029/2005JE002623.

Stauffer, M. and Butler, S.L. 2010. The Shapes of Splash-Form Tektites: Their Geometrical Analysis, Classification and Mechanics of Formation. Earth Moon Planets 107:169-196.

Taylor, S.R and Kaye, M. 1969. Genetic significance of the chemical composition of tektites: A review. Geochimica et Cosmochimica Acta 33; 1083-1100. 
Taylor, S.R. and Koeberl, C. 1994. The Origin of Tektites: Comment on a paper by J.A O'Keefe. Meteoritics 29: 739-744.

Vdovykin, G. 1972. The Canyon Diablo meteorite. Space Science Reviews 14: 758-831.

Wasson, J.T., and Kallemeyn, G.W. 2002. The IAB iron-meteorite complex: A group, five subgroups, numerous grouplets, closely related, mainly formed by crystal segregation in rapidly cooling melts. Geochimica et Cosmochimica Acta 66: 2445-2473.

Wilkison, S.L, McCoy, T.J, McCamant, J., Robinson, M., and Britt, D. 2003. Porosity and density of ordinary chondrites: Clues to the formation of friable and porous ordinary chondrites. Meteoritics \& Planetary Science 38 (10): 1533-1546. 


\section{Appendix A: Iron meteorite models}

Almost 4 dozen meteorites were modeled over the course of this study. Only a fraction of these samples were shown in the text, some of which were not shown completely. This appendix serves to provide a review of different features and shapes examined. Other samples can be seen in Figures 2.4, 3.1, and 3.4-3.7. Scales displayed are in centimeters.

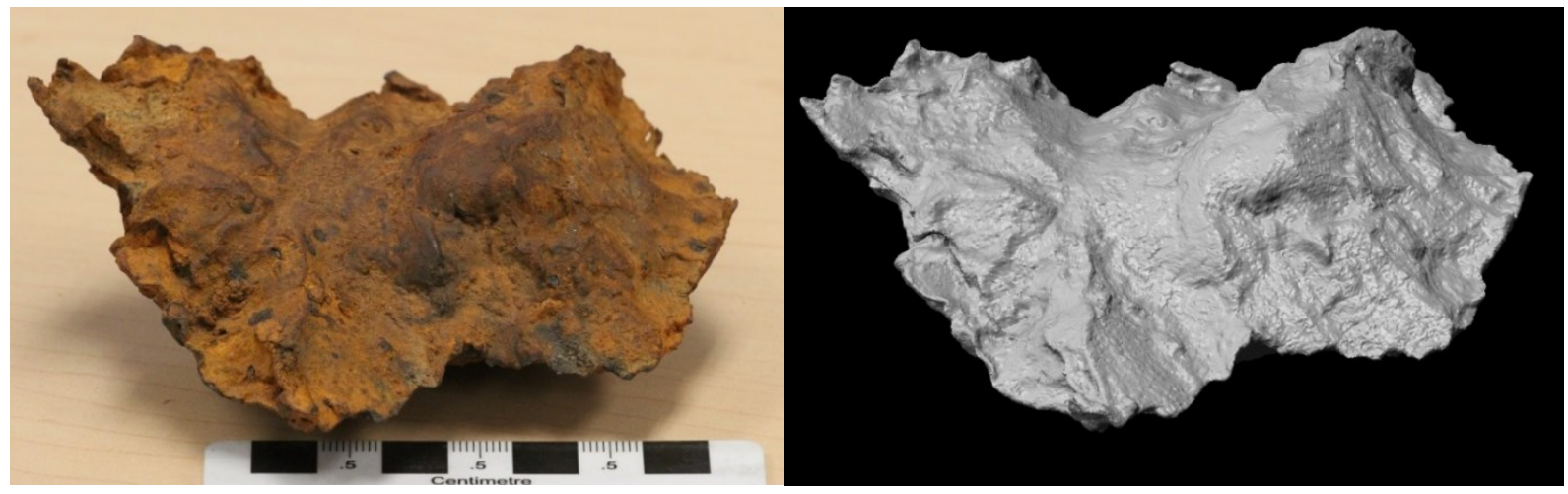

Whitecourt sample 11617/10; One of the larger samples examined, it had a shrapnel morphology and was highly coated in rust.

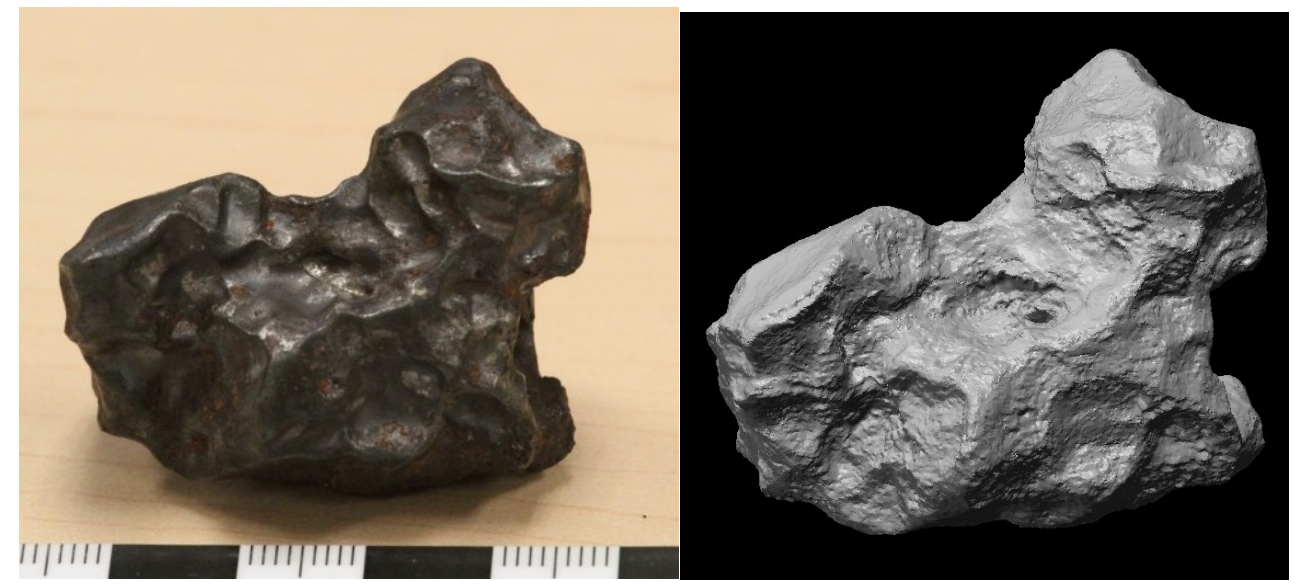

Sikhote-Alin sample T537; This sample had a number of distinct regmaglypts, and a large pit on the side.
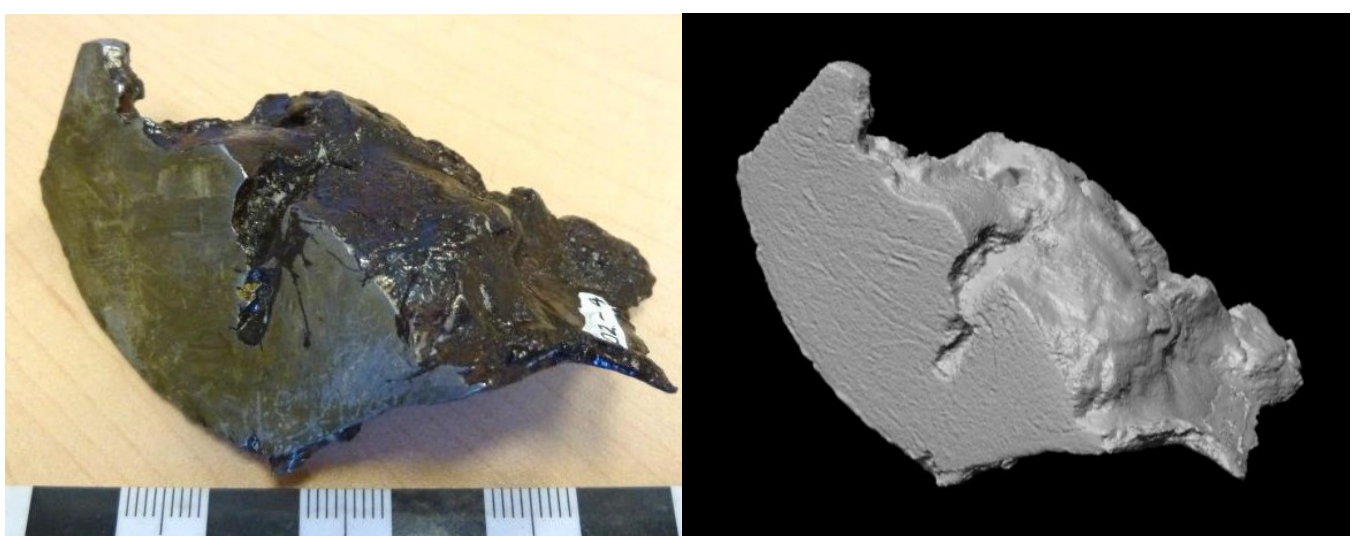

Gibeon sample 0702-4; This sample possessed a cut face, and a sail shape. 


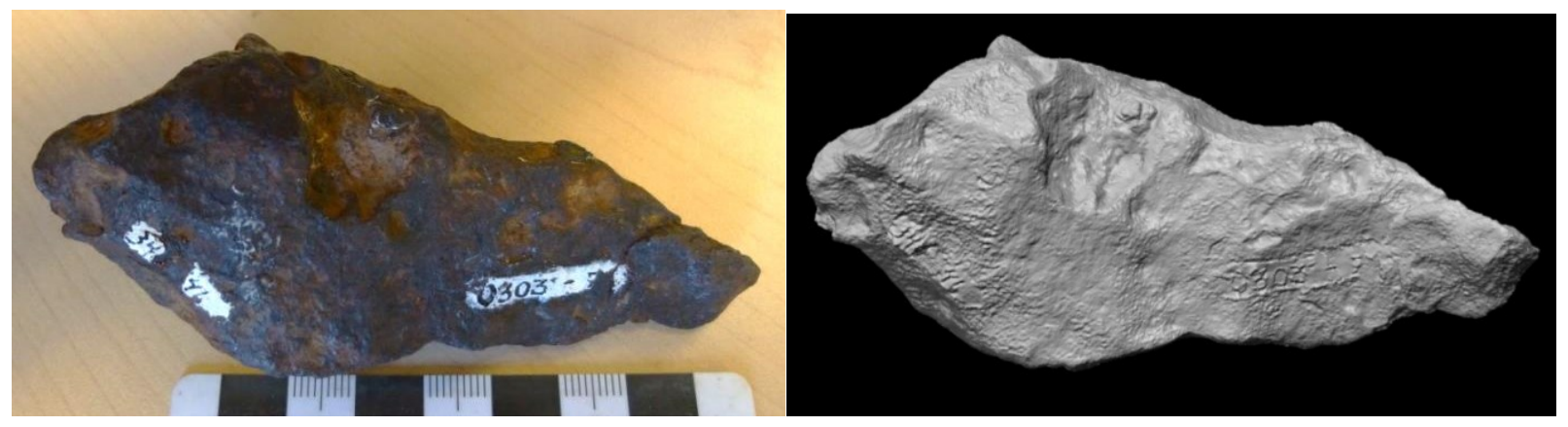

Canyon Diablo sample 303-7; This sample had a tapering shape, and a large pit on top.

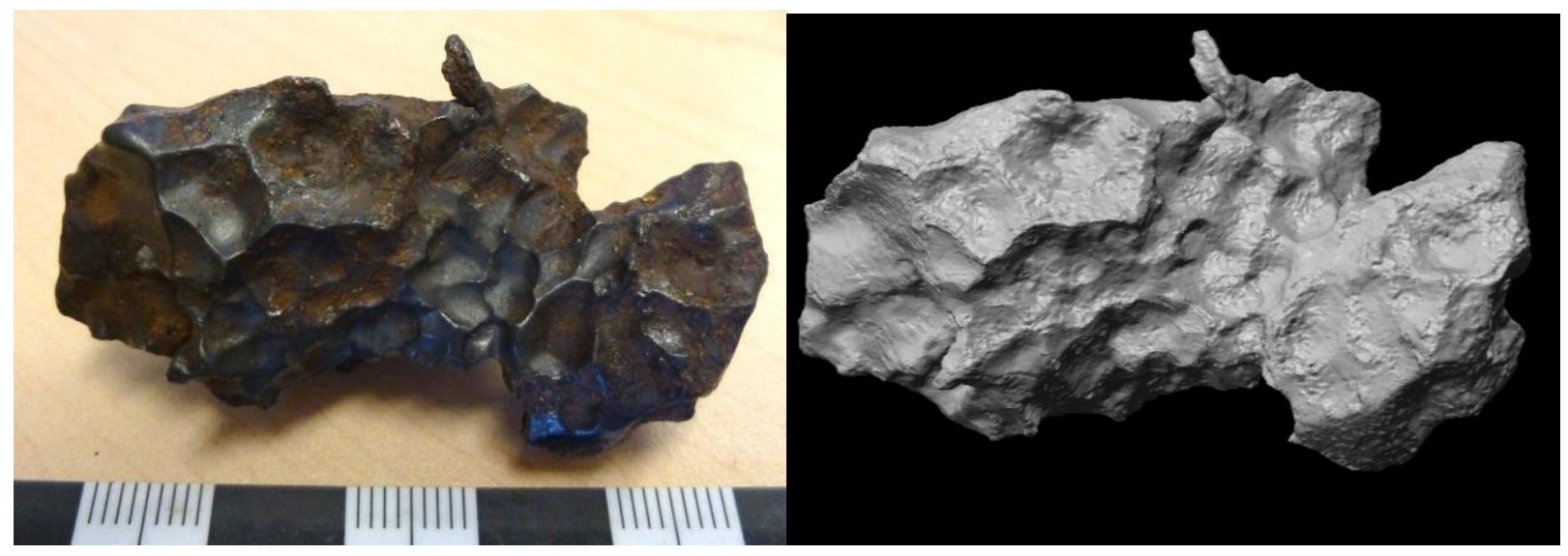

Sikhote-Alin sample T538; Displaying a number of regmaglypts, the upper surface possess an iron tendril
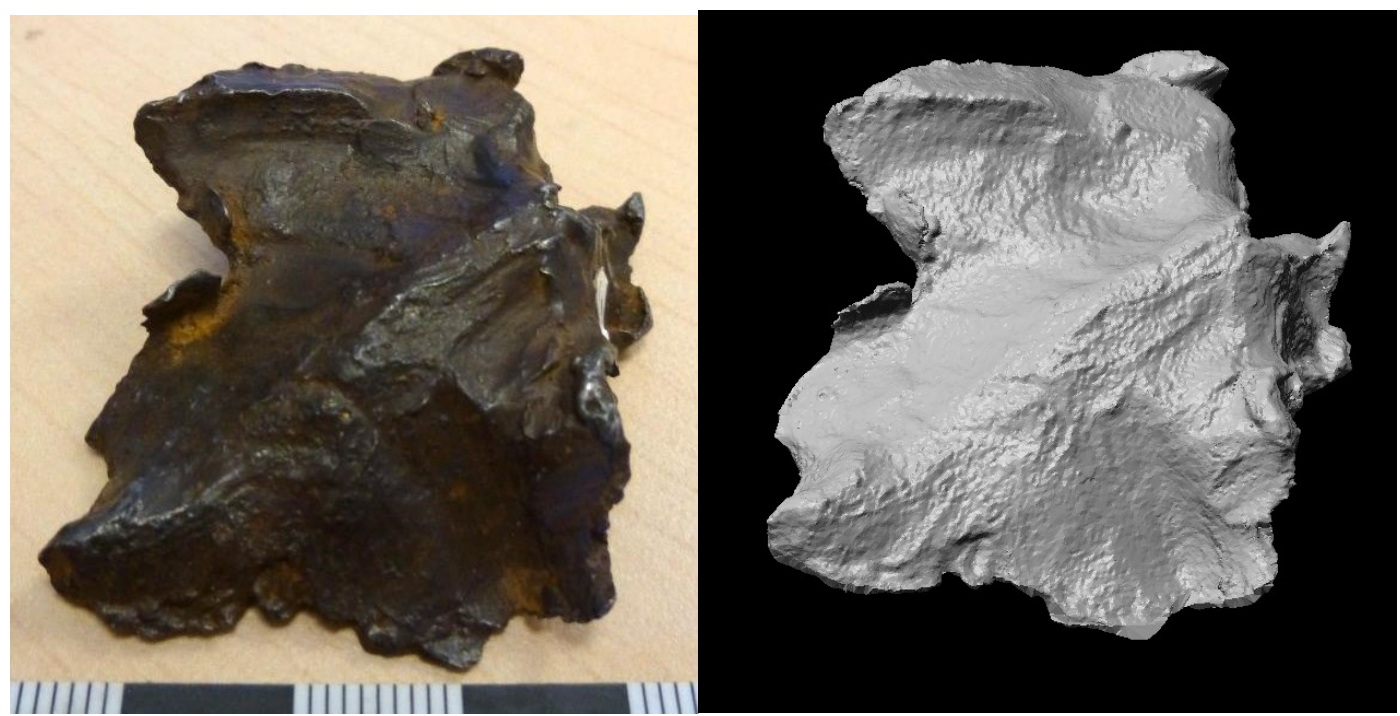

Sikhote-Alin sample T485; This sample resembled a piece of shrapnel. It has sharp edges and a flattened shape. 


\section{Appendix B: Tektite models}

11 tektites were imaged, with 10 complete models being produced. The 10 tektites are displayed below.
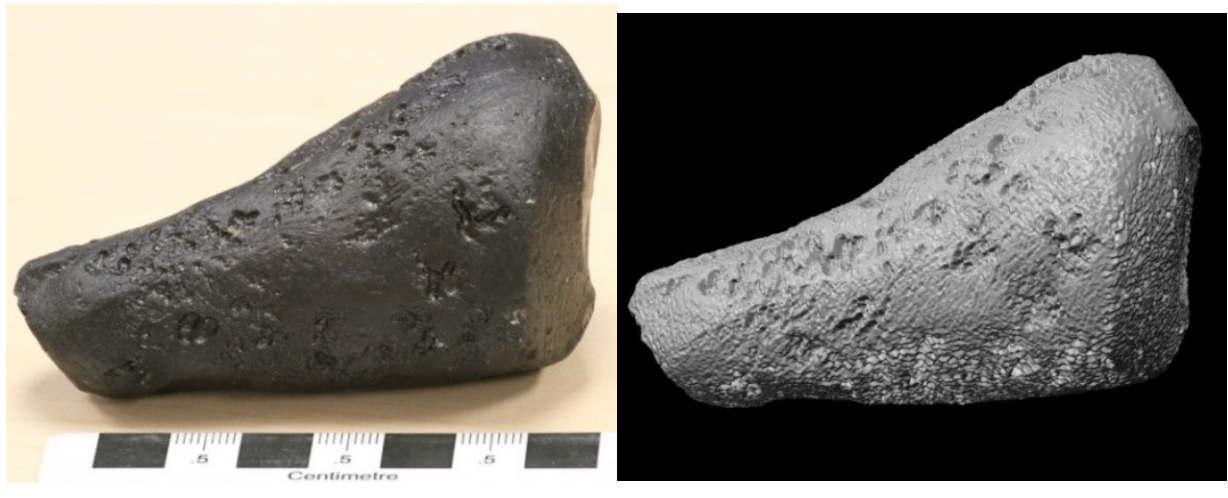

Tear drop tektite,TT1: 175.00g
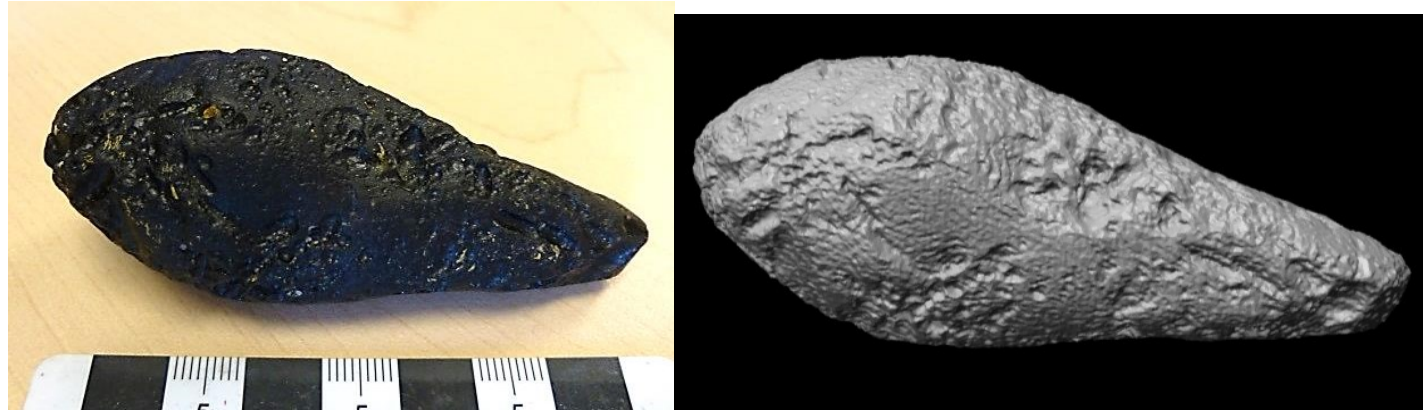

TT2: 71.53g. Of note is that abundant lechatelierite is visible on the surface of this sample

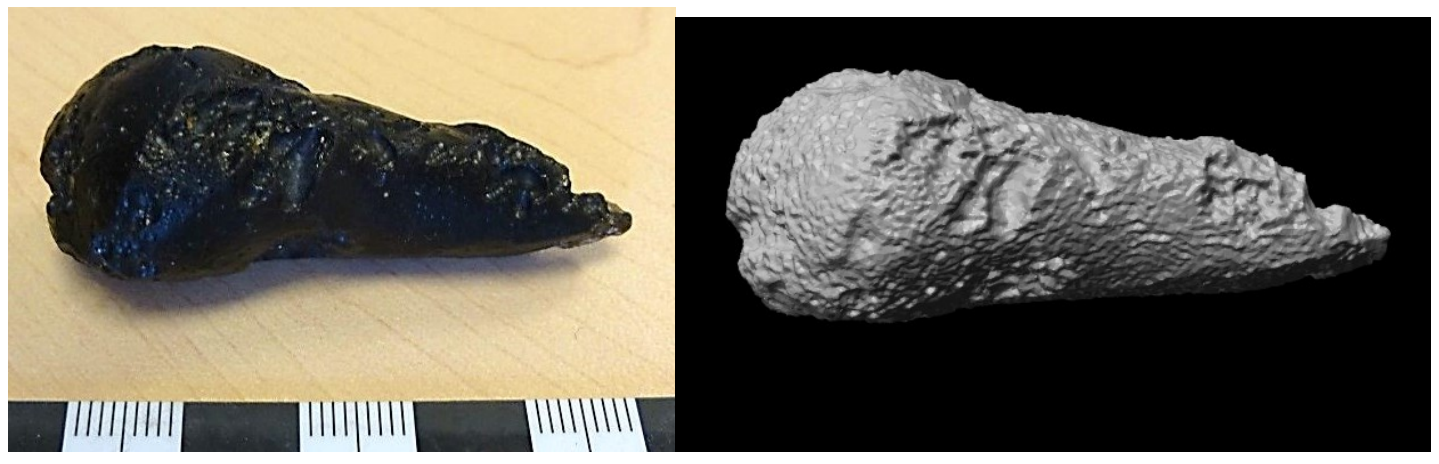

TT 4: 21.20g
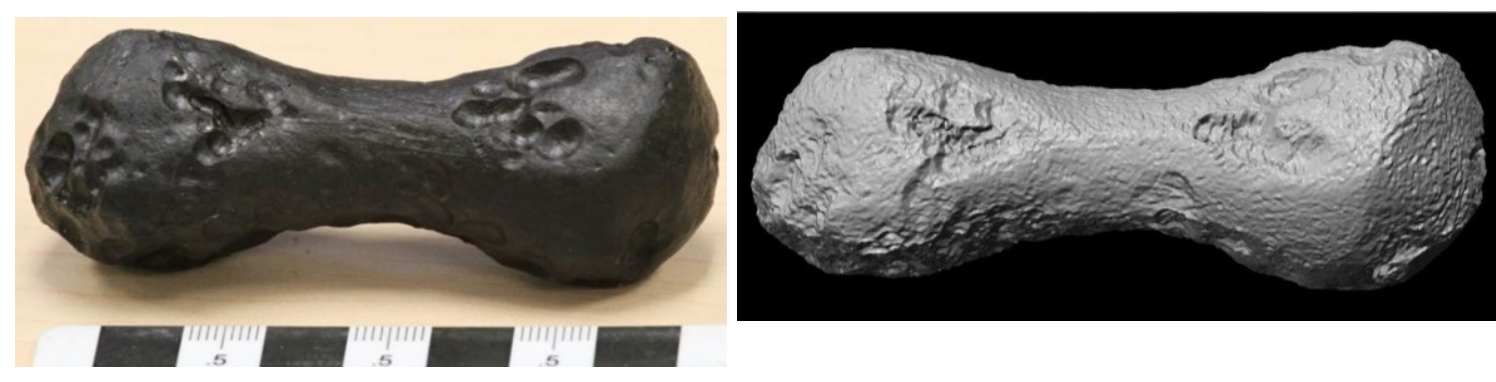

Dumbbell tektite, TB1: 72.69g 


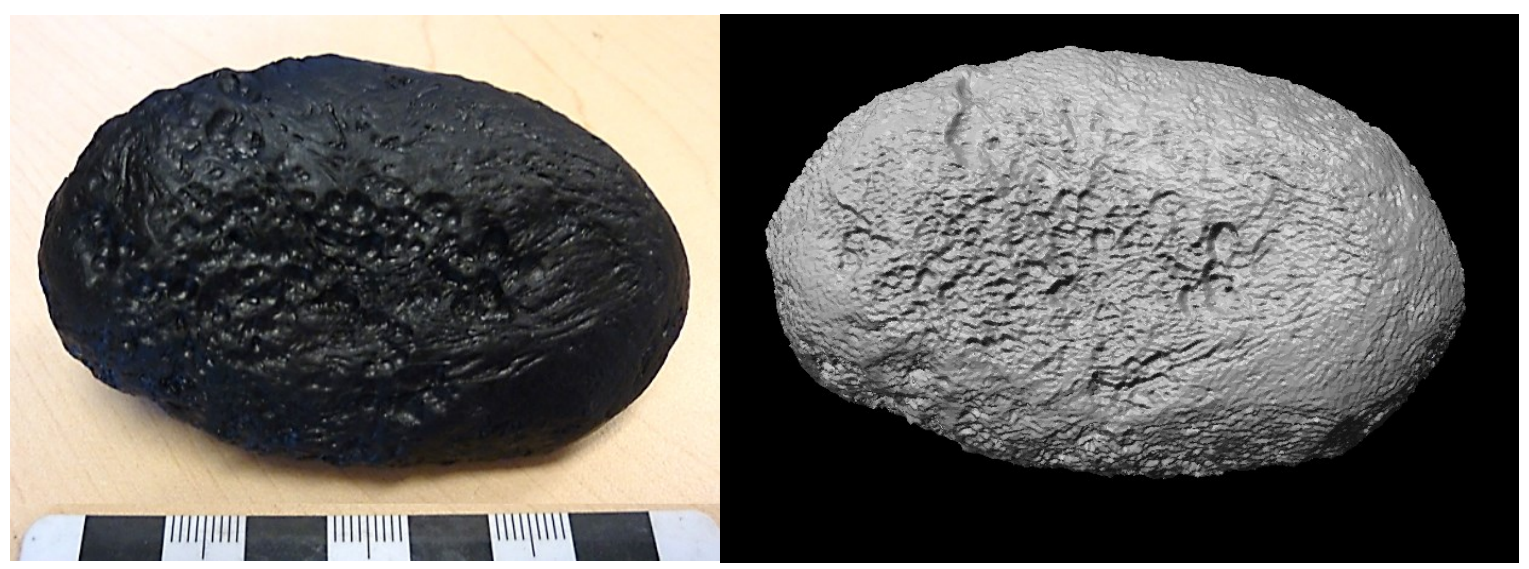

Disc tektite TD1: $150.00 \mathrm{~g}$, schlieren appear on the smooth surfaces
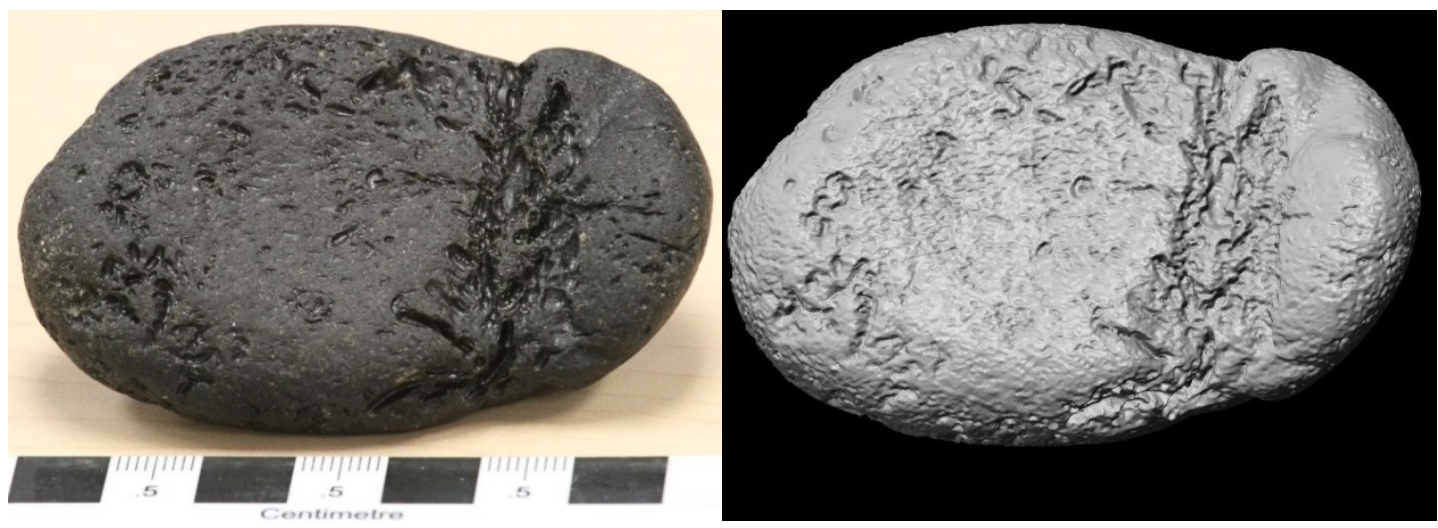

TD2: $84.25 \mathrm{~g}$

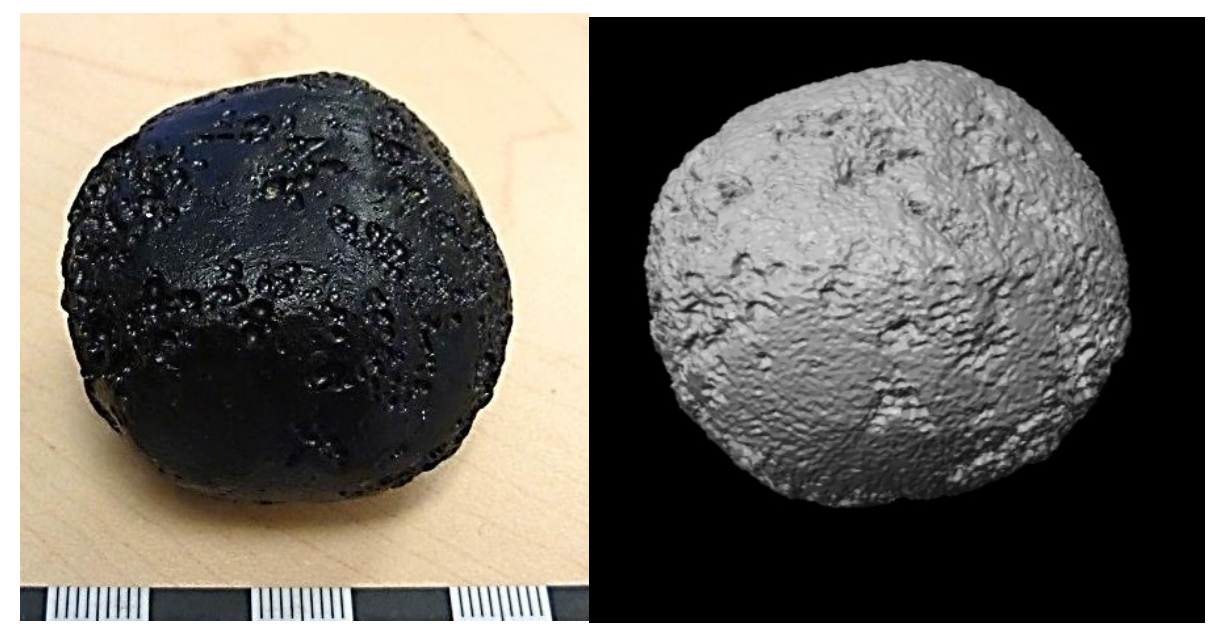

Ellipsoid tektite T01: 76.11g 


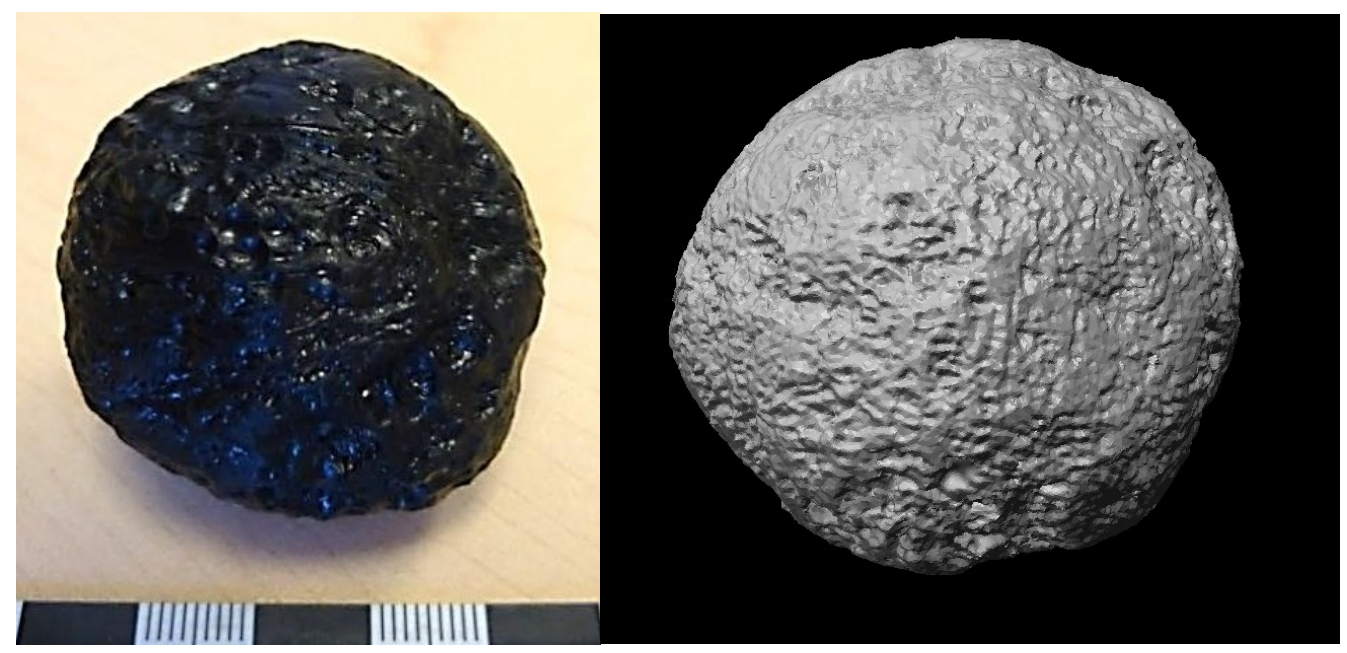

TO2: $68.00 \mathrm{~g}$

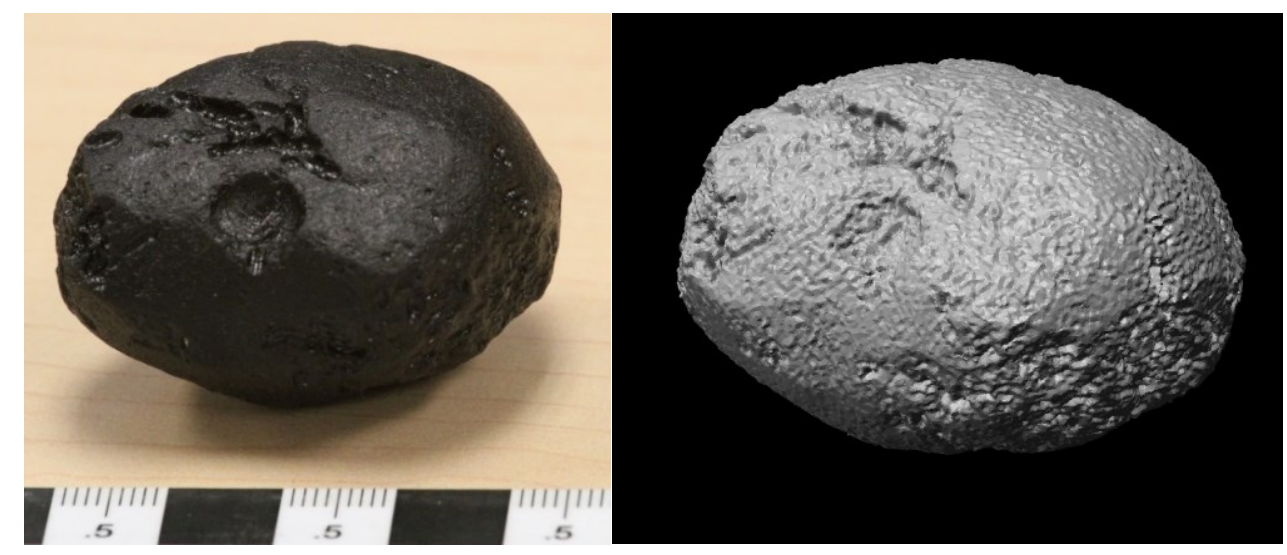

T03: $59.67 \mathrm{~g}$

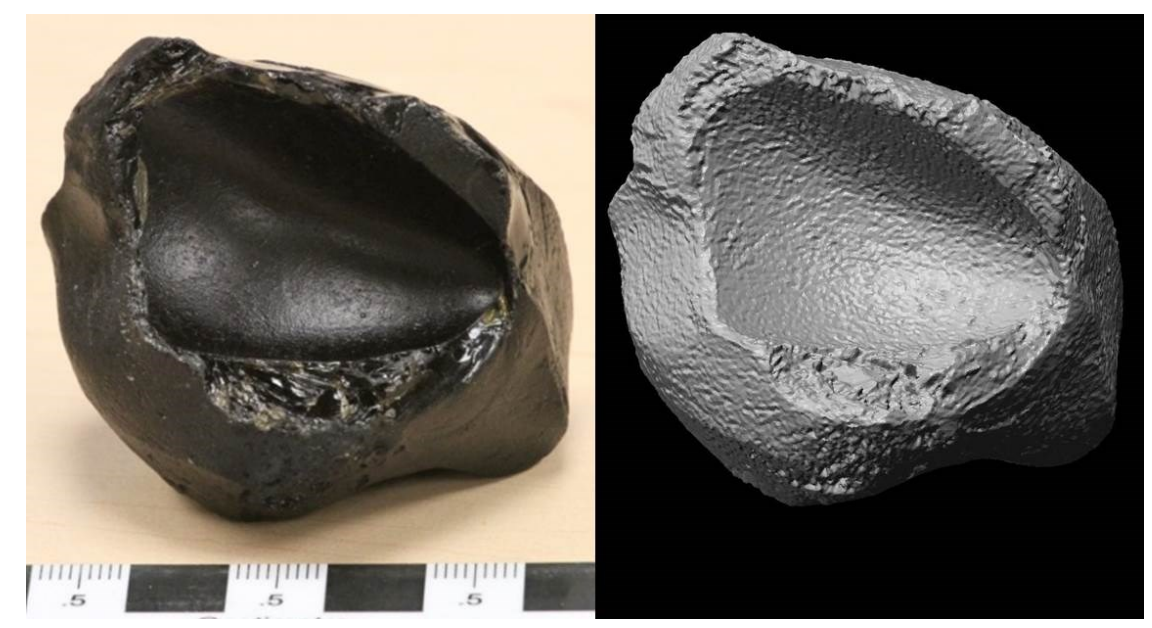

Unique tektite, TU1: $102.60 \mathrm{~g}$, this fragment was originally larger, and was broken sometime after formation, due to a large interior space. 